\title{
An Adaptive, Linear, and High Gain X-Band Gallium Nitride High Power Amplifier
}

\author{
by
}

\author{
Srdjan Najhart
}

A thesis submitted to the Faculty of Graduate and Post Doctoral

Affairs in partial fulfillment of the requirements for the degree of Master

$$
\text { of Applied Science }
$$

Ottawa-Carleton Institute for Electrical Engineering

Department of Electronics

Faculty of Engineering

Carleton University

Ottawa, Canada

(C) Srdjan Najhart, 2016 


\begin{abstract}
The work in this thesis presents the design and implementation of an MMIC reconfigurable power amplifier operating in the X-band for the purpose of creating a smart antenna system capable of adapting to performance compromising situations arising from changes in impedance due to thermal expansion and electromagnetic coupling to nearby dielectric objects. A two-stage power amplifier and three-bit reconfigurable output matching network topology is implemented in a coplanar waveguide design within a $4 \mathrm{~mm} \times 2 \mathrm{~mm}$ area using a $500 \mathrm{~nm}$ AlGaN/GaN process. Simulations show an output power of $34 \mathrm{dBm}$, with PAE of $33 \%$ and gain of $23 \mathrm{~dB}$ at the $1 \mathrm{~dB}$ compression point, and a saturated output power of $36 \mathrm{dBm}$ and peak PAE of $36 \%$, which is accomplished using an $800 \mu \mathrm{m}$ wide HEMT device. Eight discreet output impedance steps allow a tuning range corresponding to a VSWR ratio of 2.1 .
\end{abstract}




\section{Acknowledgments}

There are a number of individuals I would like to thank for their contributions, both tangible and intangible, to my research and towards the completion of this thesis. My supervisor, Dr. James Wight, has imparted to me a great deal of advice and wisdom, and provided me with the resources needed to carry out my research. My co-supervisor, Dr. Rony E. Amaya, benefited my research with his wealth of MMIC design experience and novel ideas, and has connected me with the right individuals and organizations that were instrumental in realizing my designs.

Special thanks to Nagui Mikhail, physical facilities manager at Carleton University, who was very generous and helpful in providing me with access to the software and lab equipment required for testing, and for always being on-hand and willing to help troubleshoot any technical issues. The folks at CMC Microsystems, in particular James Dietrich, for working with and accommodating me in their fabrication runs. The Department of Electronics and its staff and the University as a whole for giving me this unique and invaluable opportunity and for facilitating my research through funding and access to its facilities and resources.

Finally, and most of all I wish to thank my parents, Branko and Slavica Najhart, for their constant encouragement and support. I would never have become the person I am or had the opportunities that I have been grateful to receive, without their hard work, love and affection. 


\section{Contents}

Abstract $\quad$ ii

Acknowledgments $\quad$ iii

Contents $\quad$ vi

List of Tables vii

List of Figures $\quad$ X

List of Abbreviations $\quad$ xiii

1 Introduction 1

1.1 Radio ........................... . . . . 1

1.2 GaN Power Amplifiers at X-Band . . . . . . . . . . . . . . . 5

1.3 Adaptive Matching Networks . . . . . . . . . . . . . . . . . 6

1.4 Objectives ............................ 7

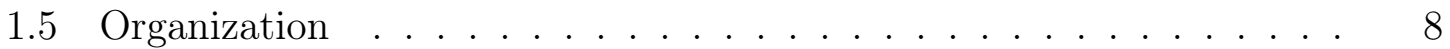

2 Background $\quad 9$

2.1 Semiconductor Materials . . . . . . . . . . . . . . . . . 9

2.2 RF Transistors . . . . . . . . . . . . . . . . . 11

2.3 Process . . . . . . . . . . . . . . . . . . 16

2.4 Small Signal Amplifiers . . . . . . . . . . . . . . . . . . 18 
2.5 Power Amplifiers . . . . . . . . . . . . . . . . . . . . . . . 24

2.5 .1 Loadline Matching . . . . . . . . . . . . . . . . . 25

2.5.2 Performance Parameters . . . . . . . . . . . . . . . . . . 28

2.5 .3 Classes of Operation . . . . . . . . . . . . . 33

2.6 Planar Waveguide Transmission Lines . . . . . . . . . . . . . . . 41

2.6 .1 Microstrip . . . . . . . . . . . . . . . . . . . . . 43

2.6 .2 Coplanar Waveguides . . . . . . . . . . . . . . . . . . 45

2.7 Programmable Tuners $\ldots \ldots \ldots \ldots$

2.8 Antennas . . . . . . . . . . . . . . . . . . . . . . . . . . 52

2.8 .1 Antenna Types . . . . . . . . . . . . . . . . . 53

2.8 .2 Antenna Detuning . . . . . . . . . . . . . . . . 55

2.9 Previous Works $\ldots \ldots \ldots \ldots \ldots \ldots$

$\begin{array}{lll}3 & \text { Design } & 63\end{array}$

3.1 Specifications $\ldots \ldots \ldots \ldots \ldots \ldots \ldots \ldots \ldots \ldots$

3.2 Power Amplifier Design . . . . . . . . . . . . . . . . . . . . . 63

3.2.1 Biasing and Matching Networks . . . . . . . . . 63

$3.2 .2 \quad$ Power Amplifier Stage Design _ . . . . . . . . . . . . . 68

3.2 .3 Driver Stage Design . . . . . . . . . . . . . . . . . . . . 74

3.3 Antenna Detuning Simulations . . . . . . . . . . . . . . . 76

3.4 Programmable Impedance Matching Network Design _ . . . . . . . 82

3.5 Layout . . . . . . . . . . . . . . . . . . . . . . 85 
4.1 Test Setup and Results . . . . . . . . . . . . . . . . . . . . 92

4.2 Sources of Instability . . . . . . . . . . . . . . . . . . 96

5 Conclusions

5.1 Results......................... . . 100

5.2 Future Work . . . . . . . . . . . . . . . . . . . . 101

$\begin{array}{ll}\text { References } & 108\end{array}$ 


\section{List of Tables}

2.1 Semiconductor Material Properties . . . . . . . . . . . . . . 9

2.2 NRC GaN500 v2 Process Parameters . . . . . . . . . . . . . 17

2.3 Comparison of Amplifier Classes . . . . . . . . . . . . . . . . . . . 38

2.4 Comparison with Previous Works . . . . . . . . . . . . . 61

3.1 Specifications . . . . . . . . . . . . . . . 63

3.2 Initial and Final Antenna Dimensions in Millimeters . . . . . . . . . . 78

3.3 Dielectric Properties of Water and Ice . . . . . . . . . . . . . . . . . . 79

3.4 Switch-Capacitor Capacitance Ratio and Q factor . . . . . . . . . 83 


\section{List of Figures}

$1.1 \quad \mathrm{RF}$ front-end . . . . . . . . . . . . . . . . . 2

1.2 Required EIRP for various receiver sensitivities. . . . . . . . . . . . 4

2.1 On-resistance and breakdown voltage of semiconductor materials . . . 11

2.2 HEMT structure . . . . . . . . . . . . . . . . . . 17

2.3 GaN 500 v2 discrete components . . . . . . . . . . . . . . . . 18

2.4 Two-port network . . . . . . . . . . . . . . . . . 20

2.5 Amplifier block diagram . . . . . . . . . . . . . . . . . . . . 22

2.6 Conjugate and loadline matching . . . . . . . . . . . . . 25

2.7 Load-pull contours . . . . . . . . . . . . . . . . . . 26

2.8 Conjugate and power matched output power . . . . . . . . . . . . . 27

2.9 Two-tone test spectrum . . . . . . . . . . . . . . . 31

2.10 Fundamental and intermodulated components . . . . . . . . . . . 32

2.11 Reduced conduction angle waveforms . . . . . . . . . . . . . . . . 34

2.12 I-V curves of amplifier classes . . . . . . . . . . . . . . . 36

2.13 Current components and conduction angle . . . . . . . . . . . . . 39

2.14 Transmission Line . . . . . . . . . . . . . . . . . . . . . . . . . . . . . 42

2.15 Microstrip waveguides . . . . . . . . . . . . . . . . 44

2.16 Electric and magnetic field distributions of a microstrip line . . . . . . 44

2.17 Coplanar waveguides . . . . . . . . . . . . . 46

2.18 Field distributions of CPW even and odd modes . . . . . . . . . . . . 47 
2.19 Switch-capacitor unit cell . . . . . . . . . . . . . . . . 50

2.20 Switch-capacitor lumped element model . . . . . . . . . . . . . . 52

2.21 Rectangular microstrip antenna . . . . . . . . . . . . . . 56

3.1 Amplifier biasing and matching networks . . . . . . . . . . . . 65

3.2 Biasing network topologies . . . . . . . . . . . . . . 66

3.3 LC and single stub matching network topologies . . . . . . . . . . 67

3.4 Stability factor and stability measure before and after addition of $2 \Omega$ series resistor. . . . . . . . . . . . . . . 70

$3.54 \times 100 \mu \mathrm{m}$ FET I-V curves. . . . . . . . . . . . . . . . . . 72

3.6 Load pull simulation showing constant power delivered and PAE contours 73

3.7 PA matching networks . . . . . . . . . . . . . . . . . 74

3.8 Driver matching networks . . . . . . . . . . . . . . 75

3.9 Two-stage PA schematic . . . . . . . . . . . . . . . 75

3.10 3D models of simulated antennas . . . . . . . . . . . . . . . . 77

3.11 Antenna return loss. . . . . . . . . . . . . . . . . . . . . . . . 78

3.12 Radome covered antenna impedance as a function of ice and water layer

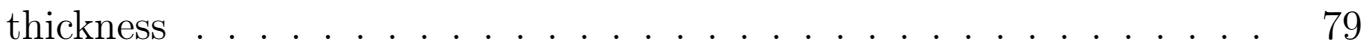

3.13 Far-field radiation patterns . . . . . . . . . . . . . 80

3.14 Antenna gain and realized gain . . . . . . . . . . . . . . . . 82

3.153 -bit programmable tuner schematic . . . . . . . . . . . 84

3.16 Simulated CPW characteristic impedance and inductance . . . . . . 86 
3.17 GaN chip layout . . . . . . . . . . . . . . . . . . . . . . . 87

3.18 PA simulated S parameters . . . . . . . . . . . . . . 88

3.19 Output power, gain and PAE vs input power . . . . . . . . . . . 89

3.20 PA simulated current and voltage waveforms . . . . . . . . . . 90

3.21 Tuner impedance states . . . . . . . . . . . . . . . . . . . 91

3.22 Water covered antenna realized gain before and after tuning . . . . . . 91

4.1 Photograph of GaN chip under microscope . . . . . . . . . . . . 92

4.2 Measured $4 \times 100 \mu \mathrm{m}$ FET I-V curves. . . . . . . . . . . . . . . 93

4.3 Measured $4 \times 50 \mu \mathrm{m}$ FET I-V curves. . . . . . . . . . . . . . . . . 94

4.4 Measured and simulated FET S parameters. . . . . . . . . . . . 95

4.5 PA measured and simulated S parameters with drain bias of $2.5 \mathrm{~V}$. . 96

4.6 Output spectrum of PA with input power of $0 \mathrm{dBm} . \ldots . . . . .97$

4.7 Driver stage FET stability factor using series and shunt resistors. . . . 98

4.8 PA S parameters with drain bias of $2.5 \mathrm{~V}$ with various mesh densities. 99 


\section{List of Abbreviations}

2DEG Two-Dimensional Electron Gas

ABS Acrylonitrile Butadiene Styrene

ADS Advanced Design System

AlGaAs Aluminum Gallium Arsenide

AlGaN Aluminum Gallium Nitride

BJT Bipolar Junction Transistor

CMOS Complementary Metal-Oxide-Semiconductor

CPW Coplanar Waveguide

EIRP Equivalent Isotropically Radiated Power

EM Electromagnetic

FEM Finite Element Method

FET Field-Effect Transistor

FSPL Free-Space Path Loss

GaAs Gallium Arsenide

GaN Gallium Nitride

GPS Global Positioning System

HBT Heterojunction Bipolar Transistor

HEMT High Electron Mobility Transistor

HFET Heterostructure Field-Effect Transistor

IC Integrated Circuit 


\begin{tabular}{|c|c|}
\hline InP & Indium Phosphide \\
\hline ITU & International Telecommunication Union \\
\hline JFET & Junction Field-Effect Transistor \\
\hline MEMS & Microelectromechanical System \\
\hline MESFET & Metal-Semiconductor Field-Effect Transistor \\
\hline MIM & Metal-Insulator-Metal \\
\hline MMIC & Monolithic Microwave Integrated Circuit \\
\hline MOSFET & Metal-Oxide-Semiconductor Field-Effect Transistor \\
\hline NRC & National Research Council \\
\hline PA & Power Amplifier \\
\hline PAE & Power Added Efficiency \\
\hline PIMN & Programmable Impedance Matching Network \\
\hline PTFE & Polytetrafluoroethylene \\
\hline PWM & Pulse-Width Modulated \\
\hline RF & Radio Frequency \\
\hline $\mathrm{Si}$ & Silicon \\
\hline $\mathrm{SiC}$ & Silicon Carbide \\
\hline SiGe & Silicon-Germanium \\
\hline SOI & Second-Order Intermodulation \\
\hline SPDT & Single-Pole Double-Throw \\
\hline SRF & Self-Resonant Frequency \\
\hline
\end{tabular}


TEM Transverse Electromagnetic

TOI Third-Order Intermodulation

VNA Vector Network Analyzer 


\section{Introduction}

This section provides a brief overview of radio frequency (RF) transceivers, the relevant modules, an explanation of the principle issue this work aims to solve, and justification of the technology and methodology used to solve it. This requires an explanation of the impedance transformation and detuning caused by dielectric bodies or temperature shifts in the vicinity of an antenna and the advantages of gallium nitride $(\mathrm{GaN})$ technology in implementing an impedance-tunable integrated circuit (IC) power amplifier (PA). The section will conclude with an outline of the contents of this thesis.

\section{$1.1 \quad$ Radio}

Electronic wireless communication systems have been employed since the late nineteenth century. Some of the first inventions included the carbon microphone invented by David Hughes in 1878 , which has resistive characteristics that vary by changes in air pressure due to sound waves, and the photophone invented by Alexander Graham Bell and Charles Summer Tainter in 1879, which was a wireless telephone that functioned by modulating light waves impinging on a voice sensitive flexible mirror [1] [2]. The mechanics of electromagnetic (EM) radiation were formulated by James Clark Maxwell c. 1861 - c. 1862, and confirmed by Heinrich Hertz in 1889. Eight years later, after the studying the work of Hertz, Guglielmo Marchese Marconi invented the first radio for long range wireless communications [1]. 
In modern times, common uses of radio include cellular phone communications, wireless networking, satellite navigation systems, and broadcasting audio. A radio communications link requires a transmitter and receiver, and often a single device will require both transmit and receive functionality in a single system known as a transceiver (Figure 1.1).

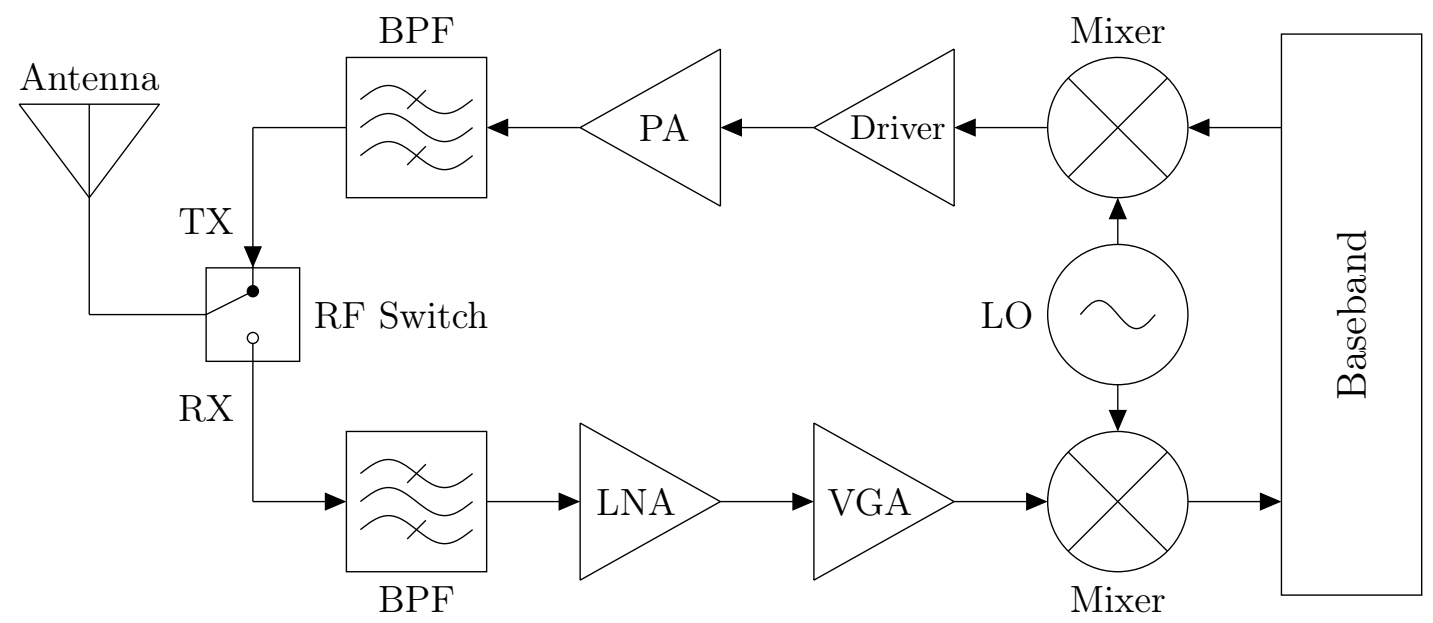

Figure 1.1: RF front-end of a simple transceiver.

The transceiver contains a radiative element known as an antenna, which is a transducer that converts electrical signals to EM radiation when transmitting and vice versa when receiving. A modulation scheme is applied to the signal by the baseband in order to encode and transmit useful information which is demodulated at the receiver. The transmit chain involves an up-conversion mixer, followed by a driver, PA, filtering, and a high frequency single-pole, double-throw (SPDT) switch or circulator to isolate it from the receive chain. The power density of the emitted signal will decrease with distance due to the spherical/isotropic spreading of the EM radiation. The reduction in power in a free-space line of sight condition is described 
by the free-space path loss (FSPL) equation.

$$
F P S L=\left(\frac{4 \pi d f}{c}\right)^{2}=\frac{P_{t} G_{t} G_{r}}{P_{r}}
$$

where $f$ is the frequency, $c$ is the speed of light in free space, $d$ is the transmitter to receiver distance, $P_{t}$ and $P_{r}$ are the transmitted and received power, respectively, and $G_{t}$ and $G_{r}$ are the transmit and receive antenna gains, respectively. Solving for $P_{r}$ shows that in order to increase the received power one must increase the antenna gains or transmit power, or lower the frequency. Achievable antenna gain within a fixed area and frequency have a direct inverse relationship because the path loss is not directly related to the frequency as EM waves are not attenuated in free-space, but rather to the aperture size of a hypothetical single element antenna with a gain of 1 , which is frequency dependent. At higher frequencies antenna elements can be more densely packed to form arrays making the gain to frequency ratio constant for a fixed physical area.

All practical antennas have some degree of directionality and do not emit power isotropically. The gain of the antenna is related to its directionality and its product with the transmit power gives an equivalent isotropically radiated power (EIRP). Taking the logarithm of (1.1) and rearranging for the EIRP gives (1.2). The EIRP is plotted as a function of distance for various receiver sensitivities assuming the receiving antenna is a typical half-wave dipole with $2.15 \mathrm{dBi}$ gain. A sensitivity of $-130 \mathrm{dBm}$ is common for a global positioning system (GPS) receiver while cellular 
phones and Wi-Fi receivers have sensitives of about $-90 \mathrm{dBm}$. Other applications such as passive RFID systems have sensitivities as low as $-20 \mathrm{dBm}$ but are not considered here.

$$
E I R P[d B m]=P_{r}[d B m]-G_{r}[d B i]+20 \log \left(\frac{4 \pi d f}{c}\right)
$$

The path loss can be very significant for many common applications using long range

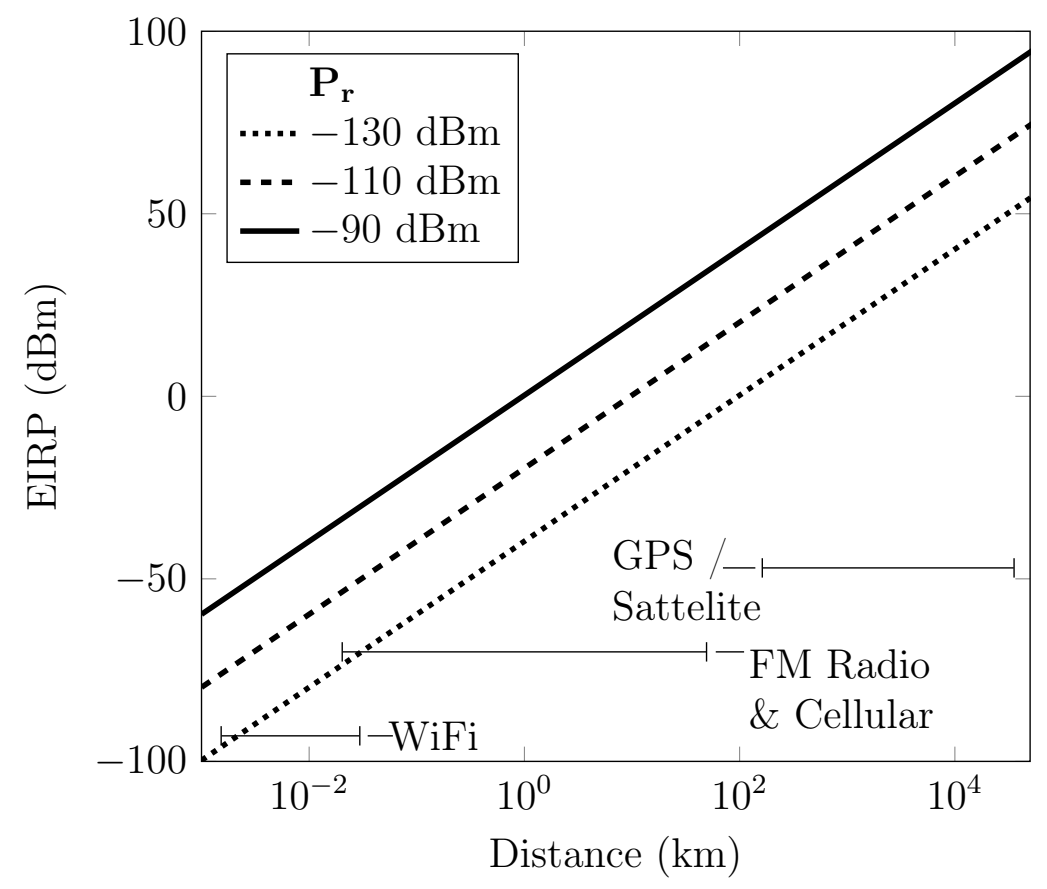

Figure 1.2: Required EIRP for various receiver sensitivities.

transmissions and especially for non-line of sight applications. Typical EIRP levels are $30-45 \mathrm{dBm}$ for basestations, $55 \mathrm{dBm}$ for GPS satellites, and $80 \mathrm{dBm}$ for FM radio stations [3]. Large phased antenna arrays are costly to build and occupy a large area, limiting the contribution of array gain to the EIRP; therefore, PAs must be able 
to output (and dissipate) a significant amount of power.

\subsection{GaN Power Amplifiers at X-Band}

Size limitations make high power density materials more desirable. While silicon ( $\mathrm{Si}$ ) has been the material of choice since the 1950s due to its high level of integrability and low cost, III-V compounds have seen use in specialized applications requiring higher speed, higher power, or lower noise. A more recent but very promising compound used in high power applications is GaN. It features a much higher band gap and critical electric field in addition to a lower permittivity, allowing it to support power densities up to a hundred times that of Si [4]. In comparison to gallium arsenide (GaAs), its most common alternative and predecessor, GaN is capable of higher switched mode efficiency, higher device output impedance resulting in low-loss matching networks due to smaller transformation ratios, and smaller die size for a given output power with a similar noise figure [5]. These features make GaN ideal for long range transmissions applications. The high power density gives it an edge in smaller devices with size constraints. However, the high breakdown voltages make it impractical in many mobile devices with supply voltages under $5 \mathrm{~V}$ but are otherwise useful for any fixed transmitter connected to the grid. One particularly attractive application area for this work is in space, aerospace, and satellite communications. A fully integrated solution is desirable because of these applications' high sensitivity to size and weight which will ultimately reduce overall costs. Apart from its high power handling capabilities 
GaN also has a high radiation resistance that makes it more durable than other technologies in environments exposed to high radiation levels.

There is a trend in adopting higher frequency bands as lower frequency bands, such as those using LTE, GSM, CDMA, Wi-Fi or Bluetooth standards, become saturated by the proliferation and increasing variety of wireless devices. This makes the Xband an attractive target for research. The X-band is designated by the IEEE to range from $8.0-12.0 \mathrm{GHz}$. In particular, the range $8.5-10.68 \mathrm{GHz}$ is assigned for radar based application for government use by the International Telecommunication Union (ITU) and currently the X-band and its sub-bands are used for weather and

climate monitoring, air and maritime vessel traffic control, defense tracking, and vehicle tracking [6]. The benefits of higher frequencies include greater bandwidth and miniaturization of monolithic microwave integrated circuit (MMIC) components whose size scales inversely with frequency. The major drawback is higher atmospheric attenuation due to oxygen and water vapour which tends to increase with frequency, and is about five times greater at $10 \mathrm{GHz}$ than at $1 \mathrm{GHz}$ [7]. There is also a greater path loss when using smaller antennas; however, this issue can be alleviated by using arrays to increase antenna gain.

\subsection{Adaptive Matching Networks}

The antenna effectively matches the PA/transmitter output impedance, usually $50 \Omega$, to the free-space impedance of $120 \pi \Omega$. Dielectric objects near the antenna have 
the ability to modify the effective impedance of the surroundings away from the free-space value. In practical applications these objects could take the form of ice buildup on the antenna, rain, or even people or packages moving near the antenna in indoor applications. Antenna performance can also be affected by thermal expansion that will detune the antenna in frequency and impedance. Therefore, in dynamic environments a programmable system capable of adaptive matching is desirable. In addition, an adaptive PA output matching network is desirable to allow multiple modes of operation giving a PA the ability to trade-off output power and efficiency for linearity and gain.

\subsection{Objectives}

A GaN process created by the National Research Council (NRC) will be used to realize a PA operating at X-band with a center frequency of $10 \mathrm{GHz}$. Simulations will be carried out using the GaN 500 v2 design kit in the Keysight Advanced Design System (ADS) environment. The PA specifications are an output power at the $1 \mathrm{~dB}$ compression point of $30 \mathrm{dBm}$ with at least $12 \mathrm{~dB}$ of gain, input and output return loss of less than $-12 \mathrm{~dB}, 30 \%$ power added efficiency (PAE) and $0.5 \mathrm{GHz}$ bandwidth. The PA shall have a programmable impedance matching network (PIMN) at the output which will need to maximize Smith chart coverage. The total die area is required to stay under $4 \mathrm{~mm} \times 2 \mathrm{~mm}$. An antenna will be designed to simulate the variation in antenna performance with tuner impedance state. This antenna will be implemented 
in a Rogers RT/duroid process rather than fully integrated in GaN to reduce cost and will be simulated using the Keysight EMPro environment.

\subsection{Organization}

The remainder of this thesis is organized into four main sections. The background section will provide the underlying theory and explanation of concepts used, as well as justifying for the design choices made later. The main topics will include a discussion on semiconductor technologies and advantages of GaN, transistor types, the GaN process used in this work's design, power amplifiers and amplifier classes, planar waveguides transmission lines, programmable tuners, and microstrip antennas, and will conclude with a survey of modern literature on the subject. The section that follows is the design. This section will use many of the concepts from the background and combine them with simulation software to realize an integrated PA with a programmable tuner, and a microstrip antenna. The third section will be the implementation. This section contains the results of the testing and experimental measurements and comparison to simulation. The last section is the conclusion and will summarize the results, placing them into context with the goal of the experiment, and will suggest areas of improvement and further research into the topic. 


\section{Background}

\subsection{Semiconductor Materials}

The choice of semiconductor material and transistor type in an integrated circuit process will impact PA performance. Some of the most important parameters related to the process are the conduction efficiency (or on-resistance), breakdown voltage, size, switching efficiency, and cost. Table 2.1 compares the material properties of $\mathrm{Si}$, silicon carbide $(\mathrm{SiC})$, GaAs, indium phosphide ( $\mathrm{InP})$, and $\mathrm{GaN}$. These are some of the common semiconductors material used in RF power applications but others exist such as silicon-germanium (SiGe), aluminum gallium arsenide (AlGaAs), and aluminum gallium nitride ( $\mathrm{AlGaN})$.

Table 2.1: Semiconductor Material Properties [8]

\begin{tabular}{ccccccc}
\hline \hline Parameter & Units & $\mathrm{Si}$ & $\mathrm{SiC}$ & $\mathrm{GaAs}$ & $\mathrm{InP}$ & $\mathrm{GaN}$ \\
\hline Band Gap, $E_{g}$ & $\mathrm{eV}$ & 1.12 & 2.86 & 1.42 & 1.34 & 3.39 \\
Critical Field, $E_{\text {crit }}$ & $\mathrm{MV} / \mathrm{cm}$ & 0.23 & 2.2 & 0.4 & 0.5 & 3.3 \\
Electron Mobility, $\mu_{n}$ & $\mathrm{~cm}^{2} / \mathrm{V} \cdot \mathrm{s}$ & 1450 & 950 & 8500 & 4000 & 800 \\
Relative Permittivty, $\epsilon_{r}$ & - & 11.7 & 9.7 & 12.9 & 14 & 8.9 \\
Thermal Conductivity, $\lambda$ & $\mathrm{W} / \mathrm{cm} \cdot \mathrm{K}$ & 1.45 & 3.5 & 0.46 & 0.68 & 1.3 \\
\hline
\end{tabular}

The band gap $\left(E_{g}\right)$ is the energy required for electrons to be promoted to the conduction band from the valence band. It is related to the strength of the chemical bonds between the atoms in the lattice structure. A higher band gap indicates lower intrinsic leakage current and higher operating temperatures [4], giving devices made in $\mathrm{GaN}$ or $\mathrm{SiC}$ a distinct advantage over traditional $\mathrm{Si}$ or GaAs based devices. The 
critical field $\left(E_{\text {crit }}\right)$ is the electric field strength required to initiate impact ionization causing avalanche breakdown and is also related to the strength of the chemical bonds between atoms in the lattice. The breakdown voltage $\left(V_{B R}\right)$, a parameter dictating the maximum power density in a process, is related to the critical field by (2.1).

$$
V_{B R}=\frac{W_{d r i f t} E_{c r i t}}{2}
$$

where $W_{\text {drift }}$ is the width of the drift region. Materials with higher critical fields have greater breakdown voltages and can achieve higher power densities as a result of a greater number of charge carriers that are depleted when the device reaches the critical point, which can be found from Gauss's law.

$$
q N_{D}=\epsilon_{r} \epsilon_{0} \frac{E_{\text {crit }}}{W_{\text {drift }}}=\epsilon_{r} \epsilon_{0} \frac{E_{\text {crit }}^{2}}{2 V_{B R}}
$$

where $q$ is the electron charge $\left(1.602 \times 10^{-9} \mathrm{C}\right), N_{D}$ is the total number of electrons in the volume, $\epsilon_{r}$ is the material relative permittivity, $\epsilon_{0}$ is the permittivity of free space $\left(8.854 \times 10^{-12} \mathrm{~F} / \mathrm{m}\right)$, and where the final result was found by combination with 2.1. This is the principal advantage of GaN processes in power conversion that, in addition to a greater critical fields, have larger drift regions than Si resulting in higher maximum power densities. The ideal on-resistance of the device is given by

$$
R_{D S, O N}=\frac{W_{d r i f t}}{\mu_{n} q N_{D}}=\frac{4 V_{B R}^{2}}{\mu_{n} \epsilon_{r} \epsilon_{0} E_{c r i t}^{3}}
$$


where $\mu_{n}$ is the electron mobility, and where the final result was found by combination with (2.1) and (2.2). Using the data from Table 2.1 curves corresponding to the lowest theoretical on-resistance of the various materials are plotted in Figure 2.1 and illustrate the advantage of $\mathrm{GaN}$ and $\mathrm{SiC}$ devices. These curves represent the limit of the technology and do not necessarily reflect the abilities of current processes, which may not be sufficiently mature to claim these values.

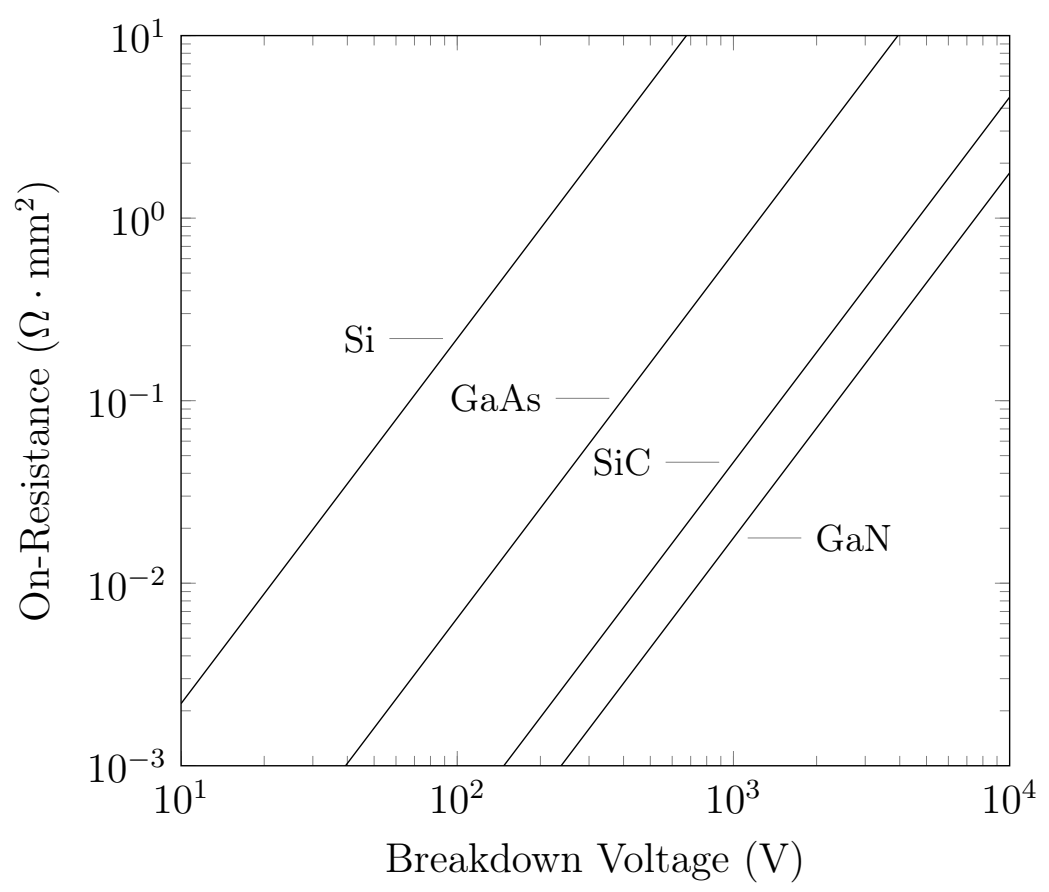

Figure 2.1: On-resistance as a function of breakdown voltage for various semiconductor materials.

\section{$2.2 \quad$ RF Transistors}

There are two basic types of solid state transistor: bipolar junction transistors (BJTs) and unipolar junction field-effect transistors (FETs). There are more exotic transistor 
topologies seen in power applications at microwave frequencies that are extensions of these basic types, especially of the FET variety, and that feature improvements such as high frequency performance, lower parasitics or higher output power.

\section{Bipolar Junction Transistors}

BJTs consist of three separate semiconductor regions, known as the emitter, base and collector, with current flowing vertically from emitter to collector. There are two possible configurations depending on the doping types (either n-type or p-type corresponding to doping resulting in excess electron or hole concentration, respectively) of these regions, known as NPN and PNP. NPN BJTs are formed by diffusing a heavily doped n-type emitter region into a p-type base region that has been diffused into a lightly doped Si substrate that forms the collector region. PNP BJTs have a similar structure but with opposite doping. BJTs in power applications typically operate with a $28 \mathrm{~V}$ bias supply and are used for frequencies below $5 \mathrm{GHz}$ [9]. They have a positive temperature coefficient that can lead to thermal instability as the current increases with increasing temperature.

\section{Heterojunction Bipolar Transistors}

Heterojunction bipolar transistors (HBTs) are BJTs that make use of compound semiconductors materials, including $\mathrm{AlGaAs} / \mathrm{GaAs}$, SiGe and $\mathrm{InP}$, in order to improve common emitter gain. The emitter is made to be as narrow as possible to decrease 
base sheet resistance and increase maximum operating frequency [9]. The junction between emitter and base is created by the heterojunction rather than by differing doping profiles as in the BJT. The higher electron mobility of the compound semiconductors also lowers the parasitics resulting in improved performance compared with Si BJTs.

\section{Field-Effect Transistors}

FETs are unipolar, voltage controlled devices. They consist of three terminals, known as the source, gate and drain. By applying a voltage between the drain and source a current channel is formed below the gate that flows laterally from the source to the drain. A voltage applied at the gate controls the current flux in the channel. The simplest type of FETs are junction field-effect transistors (JFETs) and are formed by depositing highly doped source and drain regions with ohmic contacts into a lightly doped channel, all of which share doping type; hence FETs are unipolar devices. The gate is formed by diffusing a region of opposite doping type to create a PN junction.

\section{Metal-Oxide-Semiconductor Field-Effect Transistors}

The gate contact in metal-oxide-semiconductor field-effect transistors (MOSFETs) is separated from the channel by a grown oxide layer. The channel cross-sectional area is reduced by decreasing the gate voltage (in n-type devices) until the channel is "pinched-off" and no current flows. This occurs without any gate bias in enhancement 
mode MOSFETs and at a negative gate voltage in depletion mode MOSFETs. A Si substrate is used and conveniently allows the growth of a silicon oxide layer when in the presence of oxygen to form the insulating layer above the channel. MOSFET performance suffers from parasitic capacitances and current trapping effects associated with the oxide insulator; however, it does not have the thermal stability issues seen in a BJT due to a negative temperature coefficient.

\section{Latterly Diffused MOS Transistors}

LDMOS transistors are formed by a double diffusion of source and channel dopants of opposite type to create a sufficiently short channel for high frequency applications [10]. They exhibit higher breakdown voltages than MOSFETs, and less noise and higher gain than BJTs. This makes them very useful in high power applications in the $400 \mathrm{MHz}$ to $3 \mathrm{GHz}$ frequency range.

\section{Metal-Semiconductor Field-Effect Transistors}

Metal-semiconductor field-effect transistors (MESFETs) use a semiconductor Schottky contact for the gate, eliminating the oxide layer used in MOSFETs to achieve lower parasitic capacitance between the gate and source resulting in better high-frequency performance [11]. MESFETs use III-V compound semiconductors such as GaAs with higher electron mobilities than traditional Si to further improve high-frequency performance; however, they suffer from lower breakdown voltages and lower linearity 
than MOSFETs due to the bias voltage dependent input and output capacitances.

\section{High Electron Mobility Transistors}

High electron mobility transistors (HEMTs), also known as heterostructure field-effect transistors (HFETs), are MESFETs that make use of alternating semiconductor materials such AlGaAs/GaAs or AlGaN/GaN to create charge carrier confinement below the heterojunction due to the difference in band gap energies of the semiconductors giving them better high-frequency performance. In the case of an AlGaN/GaN structure drain and source contacts are formed with a highly doped GaN layer above a doped AlGaN layer. An undoped AlGaN or AlN spacer separates the doped AlGaN layer above and undoped GaN channel layer below. Without the spacer the mobility of the two-dimensional electron gas (2DEG) would be reduced due to the interface roughness scattering mechanism between the GaN and AlGaN layer. The spacer increases carrier confinement, preventing the 2DEG from migrating into the AlGaN barrier where it is strongly dissipated [12]. Undoped GaN has a higher electron mobility and greater saturated carrier velocity giving the HEMT a higher transconductance despite the imposed barrier leading to the source and drain contacts resulting in in-

creased parasitic resistance [11]. Variations of the HEMT structure exist. pHEMT transistors circumvent the issue of deep-level trapping due to the difference in lattice constants in the heterostructure by using a very thin layer for one of the materials. mHEMT transistors represent a different solution to the same problem. A buffer layer 
is placed between the materials of different lattice constant, and is graded from top to bottom in its concentration of Indium such that it matches the lattice constant of both materials on either interface.

\subsection{Process}

The GaN process and fabrication is provided by the Government of Canada NRC. Initially, the research in this work was carried out using the NRC GaN500 v1 process, using HEMTs with $500 \mathrm{~nm}$ long gates with a $\mathrm{GaN}$ on $\mathrm{SiC}$ structure and possessing a cutoff frequency $\left(f_{T}\right)$ of $27 \mathrm{GHz}$ [13]. In the subsequent version, NRC GaN500 v2, and ultimately the process used for fabricating the design in this work, a T-shaped gate was used with greater gate finger separation allowing for a higher breakdown voltage but a reduced $f_{T}$ of $13 \mathrm{GHz}$, uncomfortably close to the X-band centered at $10 \mathrm{GHz}$. While frequency dependent performance was altered the higher breakdown voltage allowed an increase of the drain bias from 20 to $30 \mathrm{~V}$ resulting in marginally higher efficiency. Future versions (NRC GaN500 v3) will include through-wafer vias on a thinned $\mathrm{SiC}$ substrate to improve thermal performance, and thicker metal layers to reduce parasitics. In addition a $150 \mathrm{~nm}$ process is planned, offering higher $f_{T}$ for operation well into the K-band. Currently, only depletion mode devices are available but enhancement mode devices are planned for future versions.

The active device is fabricated in a HEMT structure as seen in Figure 2.2 [14]. A SiC substrate is used for its high thermal conductivity in order to efficiently dissipate 


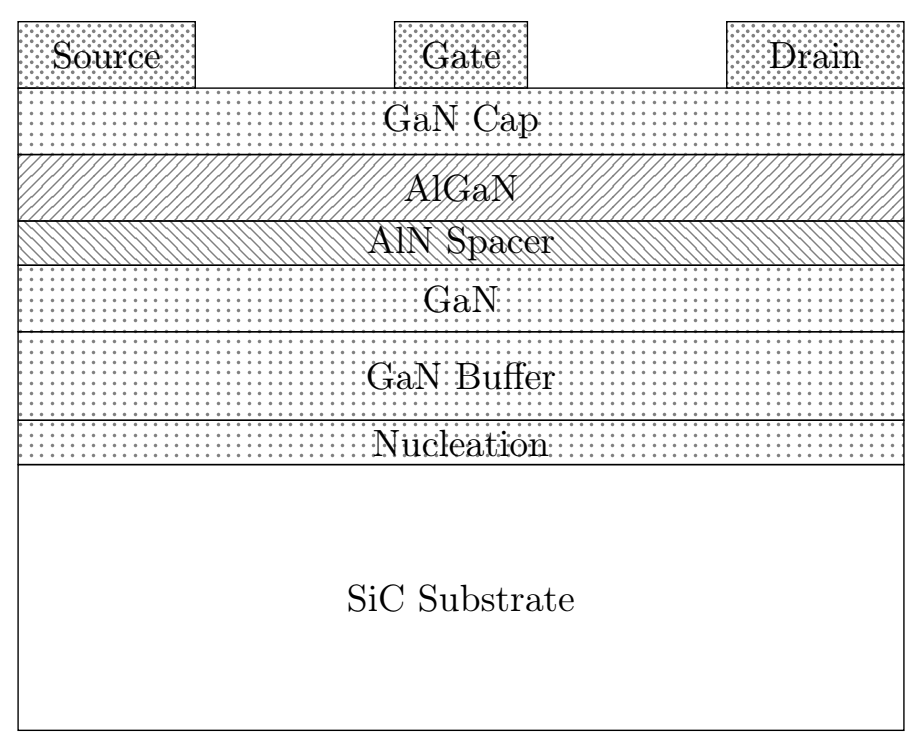

Figure 2.2: HEMT structure.

the high amounts of power used by GaN devices to prevent high channel temperatures resulting from self-heating [15]. A nucleation layer is used to grow GaN on top of the substrate. A GaN buffer layer is grown from the nucleation layer followed by the GaN channel layer. This is followed by the AlN spacer and doped AlGaN layer, finished with GaN cap and ohmic contacts for the HFET junctions. The HFET has a breakdown voltage of $100 \mathrm{~V}$ allowing a maximum DC power density of $10 \mathrm{~W} / \mathrm{mm}$, although non-linearities limit the drain bias to about $40 \mathrm{~V}$.

Table 2.2: NRC GaN500 v2 Process Parameters

\begin{tabular}{ccccc}
\hline \hline Parameter & Units & Min & Nominal & Max \\
\hline Threshold Voltage, $V_{t h}$ & $\mathrm{~V}$ & -3.6 & -4.2 & -4.6 \\
Gate-Source Voltage, $V_{G S}$ & $\mathrm{~V}$ & -8 & - & 2 \\
Saturation Current, $J_{D S S}$ & $\mathrm{~A} / \mathrm{mm}$ & 0.6 & 0.8 & 1 \\
Drain-Source Voltage, $V_{D S}$ & $\mathrm{~V}$ & 0 & - & 50 \\
Cutoff Frequency, $f_{T}$ & $\mathrm{GHz}$ & 12 & 13 & - \\
Max Oscillation Frequency, $f_{\max }$ & $\mathrm{GHz}$ & 50 & 60 & - \\
DC Bias Power, $P_{D C}$ & $\mathrm{~W} / \mathrm{mm}$ & - & - & 10 \\
\hline
\end{tabular}




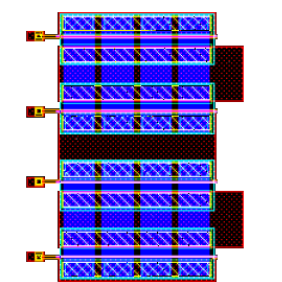

(a) HEMT.

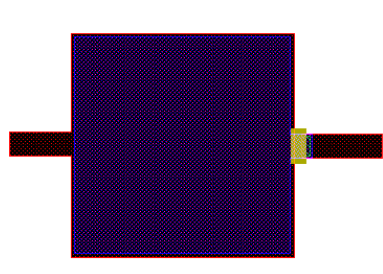

(b) MIM capacitor.

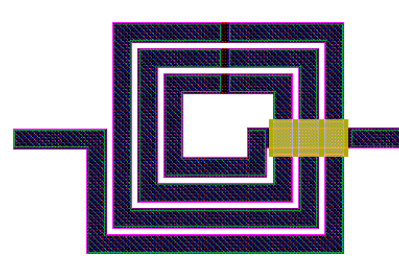

(c) Spiral inductor.

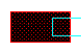

(d) Nichrome resistor.

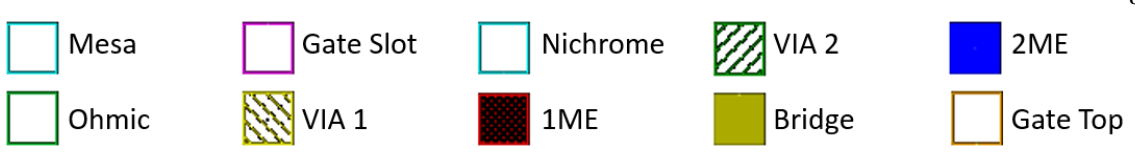

Figure 2.3: GaN 500 v2 discrete components. [14]

Some of the discrete components available in the process are seen in figure 2.3 showing the layers used to realize the components in fabrication. The NRC GaN500v2 process has two gold signal layers, $1 \mathrm{ME}$ and $2 \mathrm{ME}$, each $1 \mu \mathrm{m}$ thick with a sheet resistance of $0.027 \Omega /$ sq. Resistors are formed using nichrome and possess a sheet resistance of $50 \Omega /$ sq. The process uses its two metal layers combined with a thin insulating layer of silicon nitride to form metal-insulator-metal (MIM) capacitors with a capacitance density of $0.57 \mathrm{fF} / \mu \mathrm{m}^{2}$. Defects can occur with larger capacitors, limiting their size to a $150 \mu \mathrm{m} \times 150 \mu \mathrm{m}$ area or a capacitance of $13.5 \mathrm{pF}$. Additional process parameters are given in table 2.2 [14].

\subsection{Small Signal Amplifiers}

An ideal amplifier is represented as a gain circuit block which takes an AC signal of any frequency and multiplies its amplitude. However, the bias circuitry and matching networks of an amplifier will be tuned to a specific frequency, limiting gain outside 
of a narrow frequency band. Widebanding techniques may be used to increase the bandwidth at the expense of gain. The transistor device performance is also frequency dependent and has a maximum oscillation frequency $\left(f_{\max }\right)$ and a cutoff or unity current gain frequency $\left(f_{t}\right)$. At small input signals the device is roughly linear with constant gain; however, as the input signal is increased the device will exhibit nonlinearities and distortion as it is brought closer to saturation. A small signal amplifier is thus defined as an amplifier whose input amplitude is well below levels where nonlinearities due to clipping and saturation may occur.

To design a small signal amplifier a conjugate matching technique is used to match the transistor input and output impedance to the system reference impedance which is $50 \Omega$ in the vast majority of applications while $75 \Omega$ is sometimes used as well, mostly for cable television. $50 \Omega$ was chosen as a compromise between power handling and low loss for air-dielectric coax [16]. An impedance mismatch causes partial reflections at the junction that carries power back towards the input of the amplifier and results in lower gain at the output. The reflection coefficient $(\Gamma)$ is the proportion of the incident wave reflected back towards the source.

$$
\Gamma=\frac{Z_{L}-Z_{S}}{Z_{L}+Z_{S}}
$$

where $Z_{L}$ and $Z_{S}$ are the load and source impedances, respectively. Therefore, $\Gamma=0$ when $Z_{L}=Z_{S}$. The complex impedance is not measured directly. At lower frequencies the transmitted and reflected voltages and currents can be analyzed to determine 
the reflection coefficient; however, at microwave frequencies it becomes difficult to measure voltages and currents directly. Waveguides, such as microstrip, used to connect circuit components at high frequency do not support a TEM mode because of the unique boundary conditions imposed by the waveguide. In the case of microstrip, the inhomogeneity of the dielectric region causes the phase velocity to be different above and below the dielectric interface between substrate and air [17]. Therefore; in order to analyze reflections and mismatch losses it is necessary to introduce the concept of scattering (S) parameters. Figure 2.4 shows a 2-port network representing the input and output ports of a transistor and the associated S-parameters are given in $(2.5 \mathrm{a})-(2.5 \mathrm{~d})$.

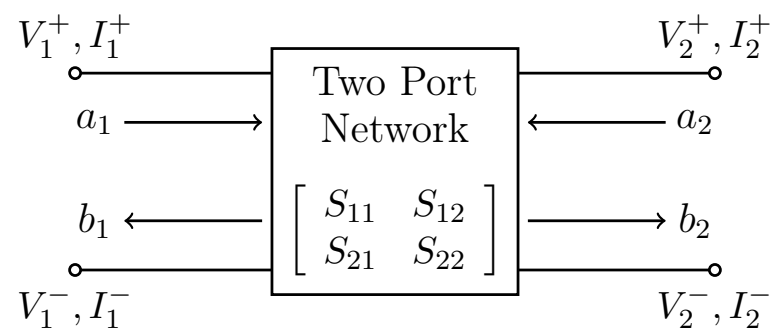

Figure 2.4: Two-port network with incident (a1, a2) and reflected $(\mathrm{b} 1, \mathrm{~b} 2)$ power waves. 
The S-parameters are related to the power waves and voltage by

$$
\begin{aligned}
& S_{11}=\frac{b_{1}}{a_{1}}=\frac{V_{1}^{-}}{V_{1}^{+}} \\
& S_{21}=\frac{b_{2}}{a_{1}}=\frac{V_{2}^{-}}{V_{1}^{+}} \\
& S_{22}=\frac{b_{2}}{a_{2}}=\frac{V_{2}^{-}}{V_{2}^{+}} \\
& S_{12}=\frac{b_{1}}{a_{2}}=\frac{V_{1}^{-}}{V_{2}^{+}}
\end{aligned}
$$

where $S_{11}$ and $S_{21}$ are the input port reflection coefficient and forward voltage gain, respectively, and $S_{22}$ and $S_{12}$ are the output port reflection coefficient and reverse voltage gain, respectively. Common source FETs can be represented by such a 2port network, with the input applied at the gate terminal and output at the drain terminal. Figure 2.5 shows a basic amplifier topology with input and output matching network blocks, omitting bias and stabilizing circuitry. For optimum power transfer it would be necessary to design input and output matching networks that transform the input and output impedances of the amplifier to the source and load impedances, respectively. At the input and output planes of the transistor this requirement can be equivalently expressed as

$$
\begin{aligned}
& S_{11}^{\prime}=\Gamma_{S}^{*} \\
& S_{22}^{\prime}=\Gamma_{L}^{*}
\end{aligned}
$$


Due to the reverse voltage gain $S_{12}$ a portion of the transfered signal will be reflected back from the load to the input. This observation is captured in the second term of (2.7a), and a similar statement may be made about the output reflection coefficient, resulting in $(2.7 \mathrm{~b})$.

$$
\begin{aligned}
& S_{11}^{\prime}=S_{11}+\frac{S_{21} S_{12} \Gamma_{L}}{1-S_{22} \Gamma_{L}} \\
& S_{22}^{\prime}=S_{22}+\frac{S_{21} S_{12} \Gamma_{S}}{1-S_{11} \Gamma_{S}}
\end{aligned}
$$

Given the S-parameters from measurement or simulation (2.7) can be used to solve

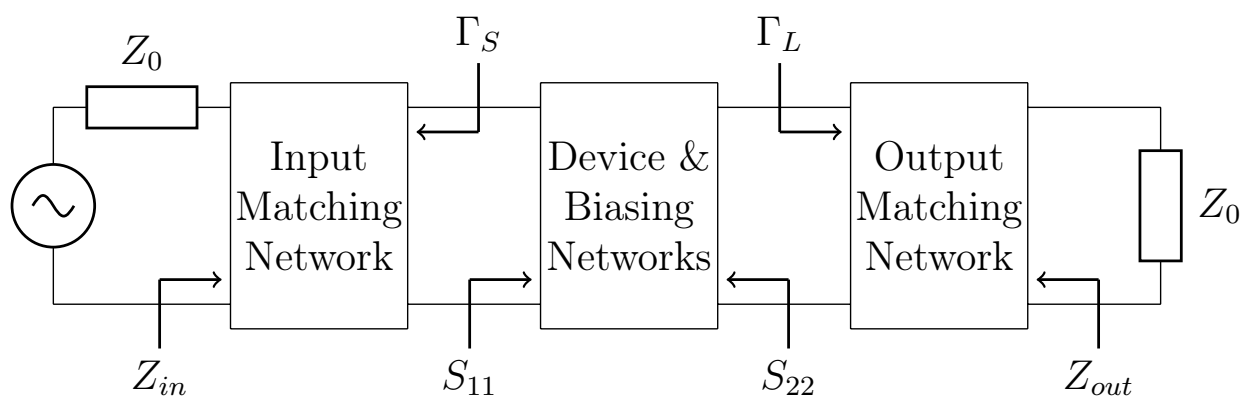

Figure 2.5: Amplifier block diagram.

for the reflection coefficients $\Gamma_{S}$ and $\Gamma_{L}$.

$$
\begin{aligned}
\Gamma_{S} & =\frac{B_{1} \pm \sqrt{B_{1}^{2}-4\left|C_{1}\right|^{2}}}{2 C_{1}} \\
\Gamma_{L} & =\frac{B_{2} \pm \sqrt{B_{2}^{2}-4\left|C_{2}\right|^{2}}}{2 C_{2}}
\end{aligned}
$$


where,

$$
\begin{array}{r}
B_{1}=1+\left|S_{11}\right|^{2}-\left|S_{22}\right|^{2}-|\Delta|^{2} \\
B_{2}=1-\left|S_{11}\right|^{2}+\left|S_{22}\right|^{2}-|\Delta|^{2} \\
C_{1}=S_{11}-\Delta S_{22} * \\
C_{2}=S_{22}-\Delta S_{11} *
\end{array}
$$

and where $\Delta=S_{11} S_{22}-S_{12} S_{21}$. Numerous matching techniques can then be used to transform the input and output impedances to realize the necessary reflection coefficients. Transformers may be used; however, they are difficult to realize in planar transmission lines due to their low coupling coefficients, and an additional reactive element is still needed to resonate out the transistor output capacitance. Stub matching is easy to realize in planar circuits but can occupy significant area at lower frequencies. A simple alternative, and the most commonly used matching network, is the L-section or LC network consisting of an inductor and capacitor that can be arranged in four possible orientations (two low-pass and two high-pass) and which can be used to match between any two complex impedances. This match is narrowband in nature which is especially a concern when matching between very low and very high impedances requiring the use of very small series capacitors and shunt inductors or very large series inductors and shunt capacitors. Switching from a low-pass to highpass network or vice-versa may provide more practical element values and increased bandwidth; however, low-pass networks are more desirable due to their harmonic 
attenuating and noise characteristics. Cascaded L-section networks with an intermediary characteristic impedance equal to the geometric mean of the adjacent network characteristic impedances may be used to increase the bandwidth of the match at the expense of added parasitics and layout area.

A conjugate matched amplifier will give the highest possible gain for a small-signal input. As the drive levels increase the transistor will begin to experience compression as it reaches its maximum output current or voltage ratings resulting in nonlinear behaviour. In order to maximize output power $\left(P_{\text {out }}\right)$ a loadline match is preferred.

\subsection{Power Amplifiers}

PAs are an integral component of most wireless transmission systems and is used on the transmitter side to drive an antenna. In contrast to small signal and low-noise amplifiers, PAs are impedance matched for power, which in some instances may result in a very nonlinear and distorted output signal. All transistors have a current carrying and breakdown voltage threshold, resulting in a maximum power output limit, known as the saturation point. PAs are often pushed near, but short of, this limit in attempt to strike a compromise between output power and linearity. There are a number of amplifier topologies or classes of operation, with varying degrees of linearity; however, efficiency and gain need to be sacrificed for high linearity, and vice versa. The design and class of operation will ultimately depend on the specifications and the application. For example, highly efficient switching PAs may not be suitable 
for high-fidelity audio applications requiring faithful sound reproduction due to distortion from their nonlinear nature of operation but may be adequate for RF base stations requiring high efficiency designs.

\subsubsection{Loadline Matching}

Assuming that the reactive component of the impedances are resonated out, the output of the amplifier may be modeled with a current source generator with output resistance $R_{\text {gen }}$ and output voltage $V_{\text {out }}$ driving a load resistance $R_{\text {load }}$ as seen in Figure 2.6 and where it has been assumed that $R_{\text {gen }}>>R_{\text {load }}$ for the loadline match.

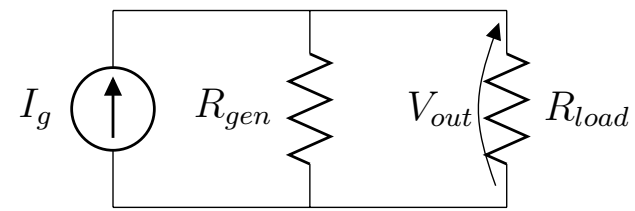

(a) FET current source model.

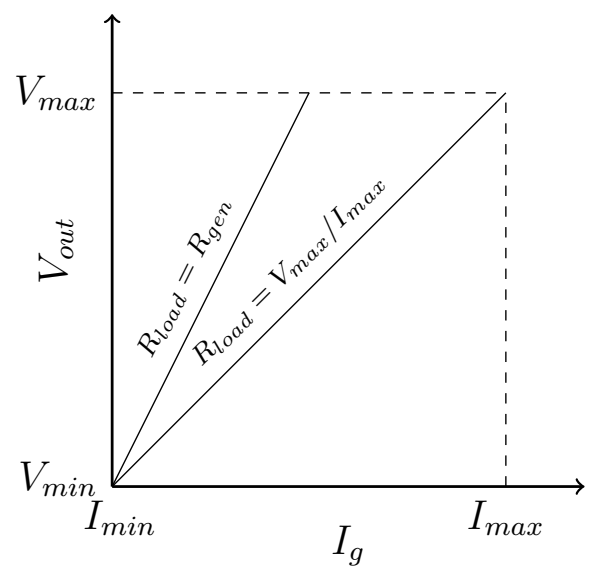

(b) Conjugate and loadline match.

Figure 2.6: Transistor output voltage and current under conjugate and loadline matching.

$$
R_{o p t} \approx \frac{V_{\max }-V_{\min }}{I_{\max }-I_{\min }}
$$


In practical PAs nonlinearities will result in an unpredictable shift of the optimal impedance and may have a reactive component. To find the optimal load impedance an empirical technique known as a load-pull is used, whereby the load impedance is swept across a range of real and imaginary value while measuring output power and efficiency. The loadline prediction may be used an as initial guess to provide a center value around which the load impedance will be swept. The results are plotted on a Smith chart as in Figure 2.7 and show constant power (or gain) and efficiency contours with the center representing the maximum value and the optimum load impedance point. The input of the amplifier is conjugately matched; however, as practical amplifiers are not unilateral (i.e. $S_{12} \neq 0$ ) the output matching network will shift the optimal impedance point at the input and vice-versa. The load-pull followed by input conjugate match process can be re-iterated until convergence is observed.

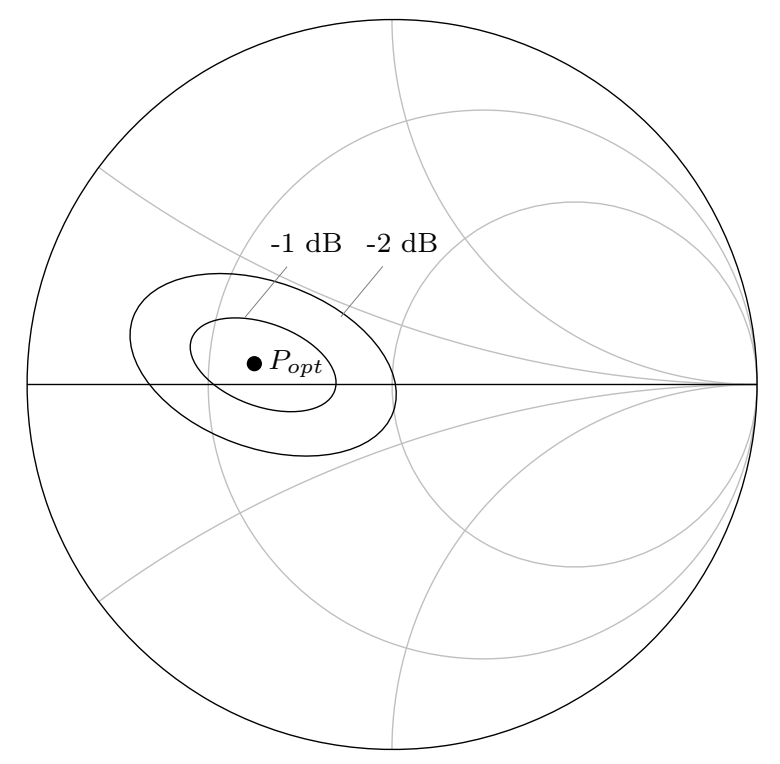

Figure 2.7: Load-pull data showing constant power contours. 
A conjugate match provides maximum gain, while a loadline match provides maximum power. This distinction is illustrated in Figure 2.8, showing a higher small signal gain but heavier compression at higher input drive levels. PA linearity and output power performance is most often quoted at the $1 \mathrm{~dB}$ compression point where the gain is $1 \mathrm{~dB}$ lower than the small signal value. Although the PA saturation point is some decibels higher the output signal will be significantly distorted if driven to this level.

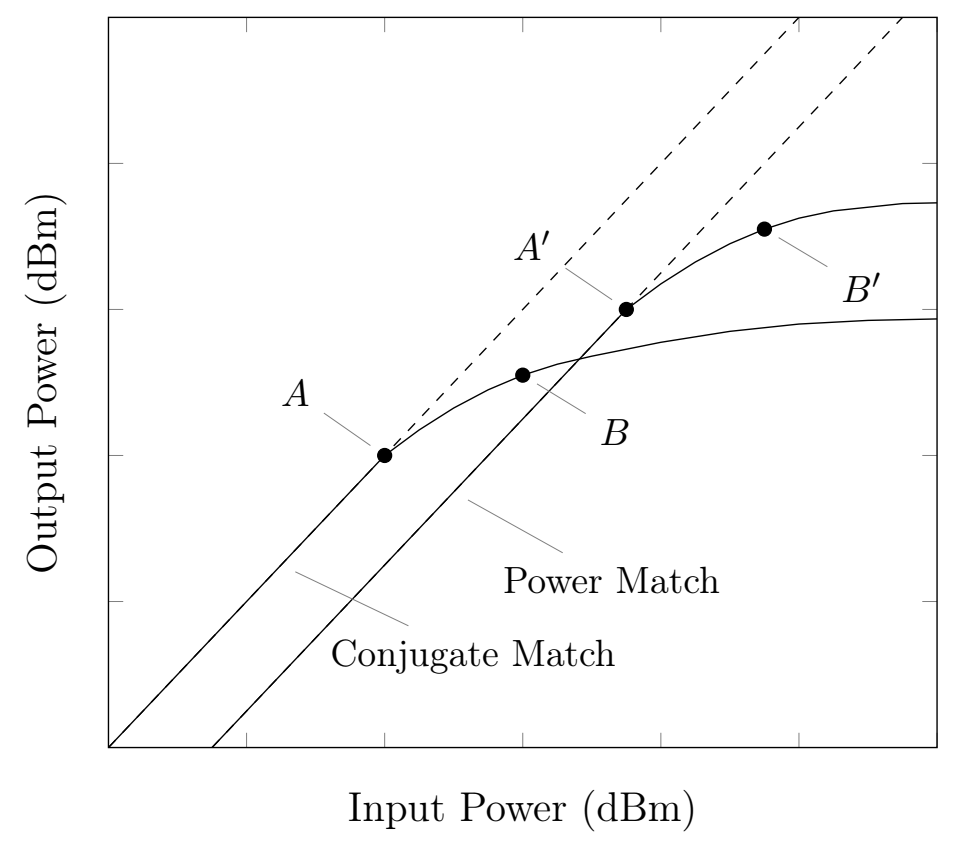

Figure 2.8: Output power curve comparison for conjugate and power matched amplifiers, indicating maximum linear points (A, $\left.\mathrm{A}^{\prime}\right)$ and $1 \mathrm{~dB}$ compression points $\left(\mathrm{B}, \mathrm{B}^{\prime}\right)$.

From figure 2.8 one can make the following observations: A conjugate match results in higher gain $\left(P_{\text {out }}-P_{\text {in }}\right)$, a power or loadline match has a higher $1 \mathrm{~dB}$ compression point, and both behave linearly for sufficiently small signals. 


\subsubsection{Performance Parameters}

\section{Gain}

Numerous power gain definitions exist for amplifiers that mostly differ on the choice of the plane of reference of the device. These include; the transducer power gain $\left(G_{T}\right)$, the maximum available power gain $\left(G_{A}\right)$, and the available power gain $\left(G_{P}\right)$ [8].

$$
\begin{aligned}
G_{T} & =\frac{P_{\text {out }}}{P_{a v s}} \\
G_{A} & =\frac{P_{a v n}}{P_{a v s}} \\
G_{P} & =\frac{P_{\text {out }}}{P_{\text {in }}}
\end{aligned}
$$

where $P_{\text {out }}$ is the output power or the power delivered to the load, $P_{\text {avs }}$ is the power available from the source, $P_{\text {avn }}$ is the power output of the device network, and $P_{\text {in }}$ is the input power to the device network.

The most commonly used is the transducer power gain or simply power gain and is can be written in terms of the S-parameters and reflection coefficients as

$$
G_{T}=\frac{\left|S_{21}\right|^{2} \cdot\left(1-\left|\Gamma_{S}\right|^{2}\right) \cdot\left(1-\left|\Gamma_{L}\right|^{2}\right)}{\left|\left(1-S_{11} \Gamma_{S}\right) \cdot\left(1-S_{22} \Gamma_{L}\right)-S_{12} S_{21} \Gamma_{S} \Gamma_{L}\right|^{2}}
$$


If $S_{21}=0$ the transistor is a unilateral device with no internal feedback.

$$
G_{T U}=\frac{\left|S_{21}\right|^{2} \cdot\left(1-\left|\Gamma_{S}\right|^{2}\right) \cdot\left(1-\left|\Gamma_{L}\right|^{2}\right)}{\left|\left(1-S_{11} \Gamma_{S}\right) \cdot\left(1-S_{22} \Gamma_{L}\right)\right|^{2}}=G_{S}\left|S_{21}\right|^{2} G_{L}
$$

where $G_{S}$ and $G_{L}$ are the gain (or loss) of the input and output matching networks, respectively. Therefore, the unilateral gain is a function of the forward voltage gain of the transistor and gain of the matching networks.

$$
\begin{aligned}
G_{S} & =\frac{1-\left|\Gamma_{S}\right|^{2}}{\left|1-S_{11} \Gamma_{S}\right|^{2}} \\
G_{L} & =\frac{1-\left|\Gamma_{L}\right|^{2}}{\left|1-S_{22} \Gamma_{L}\right|^{2}}
\end{aligned}
$$

Unilateral gain is; therefore, maximized when $\Gamma_{S}=S_{11}^{*}$ and $\Gamma_{L}=S_{22}^{*}$.

\section{Linearity}

All practical amplifiers will suffer from distortion caused by nonlinearities. The transfer function of a nonlinear system without hysteresis can be modeled as a power series [18]

$$
v_{\text {out }}=k_{1} v_{\text {in }}+k_{2} v_{\text {in }}^{2}+k_{3} v_{\text {in }}^{3}+\ldots
$$

where $v_{\text {in }}$ and $v_{\text {out }}$ are the time-dependent input and output voltages of the system, respectively, and $k_{i}=k_{1}, k_{2}, k_{3} \ldots$ are complex coefficients. For linear amplifiers $k_{2}=$ 
$k_{3} \ldots=0$ and $(2.15)$ is reduced to

$$
v_{\text {out }}=k_{1} v_{\text {in }}
$$

where $k_{1}$ is the (small signal) gain For weakly nonlinear amplifiers $k_{1}>k_{2}>k_{3} \ldots$. The terms above the third order power are often sufficiently low compared to the fundamental that they are ignored in most linearity analyses. There are a number of linearity tests and measurements that can be performed on a system. Most common are the one-tone and two-tone tests. For the one-tone test the input signal takes the form

$$
v_{i n}=A \cos \omega t
$$

where $A$ is the amplitude, and $\omega=2 \pi f$ is the angular frequency. Substituting this into (2.15) and ignoring terms above the third order gives

$$
v_{\text {out }}=\frac{1}{2} k_{2} A^{2}+\left(k_{1} A+\frac{3}{4} k_{3} A^{3}\right) \cos \omega t+\frac{1}{2} k_{2} A^{2} \cos 2 \omega t+\frac{1}{4} k_{3} A^{3} \cos 3 \omega t
$$

The above equation is arranged in order to show the DC component, fundamental, and second and third harmonics. Were higher order terms included, higher harmonics would also appear. A large fundamental component and; hence, high gain at the fundamental, is desirable. The nonlinear fundamental gain is the ratio of the 
fundamental component to the input signal.

$$
G_{N L}=\frac{k_{1} A+\frac{3}{4} k_{3} A^{3}}{A}=k_{1}+\frac{3}{4} k_{3} A^{2}
$$

A simple measure of the nonlinearity of an amplifier is the $1 \mathrm{~dB}$ compression point output power $\left(P_{1 d B}\right)$, the point at which the gain of the amplifier is reduced by $1 \mathrm{~dB}$ from the small signal level. A $1 \mathrm{~dB}$ drop is equivalent to a 0.8913 power ratio.

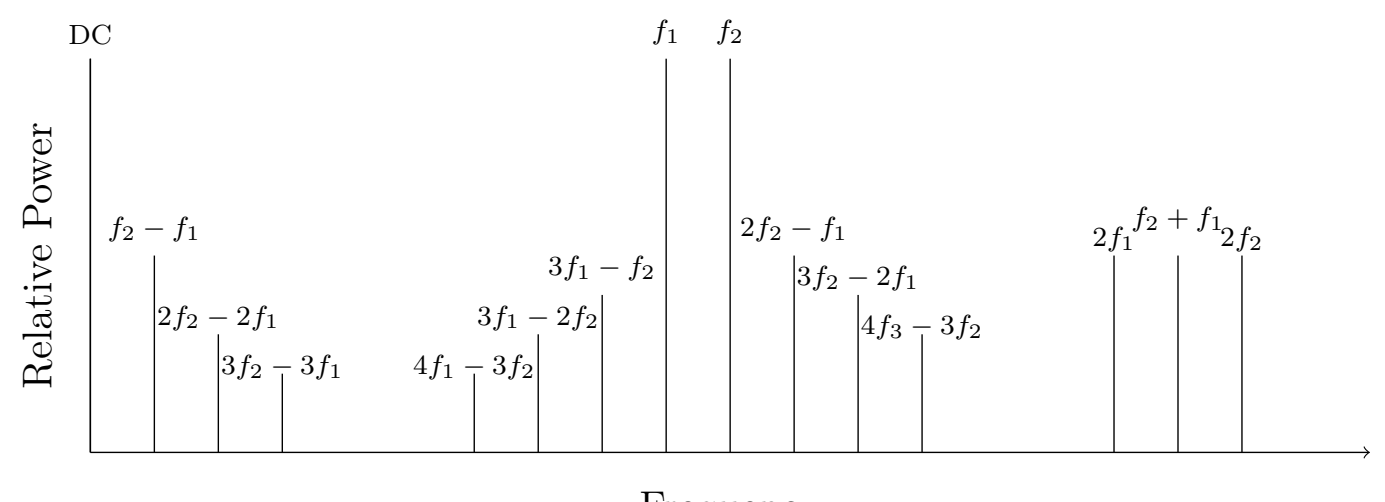

Figure 2.9: Output spectrum of two-tone test.

In a two-tone test the input voltage signal is of the form

$$
v_{\text {in }}=B\left[\cos \omega_{1} t+\cos \omega_{2} t\right]
$$

where $\omega_{1}=2 \pi f_{1}$ and $\omega_{2}=2 \pi f_{2} . \quad f_{1}$ and $f_{2}$ are equally spaced above and below the frequency of interest such that $f_{1}=f_{0}-\Delta f$ and $f_{2}=f_{0}+\Delta f$. (2.20) can be substituted into (2.15) and expanded up to the third order terms in a manner similar to the one-tone test. The expansion gives a number of terms consisting of the 
DC component, fundamentals, harmonics, and intermodulation (mixing) products. If $f_{1}$ and $f_{2}$ are closely spaced and a bandpass filter is applied at the output, the DC component, higher harmonic components $\left(2 \omega_{1}, 2 \omega_{2}, 3 \omega_{1}\right.$, and $\left.3 \omega_{2}\right)$, second-order intermodulation (SOI) product $\left(\omega_{1}+\omega_{2}\right)$, and higher third-order intermodulation (TOI) products $\left(2 \omega_{1}+\omega_{2}\right.$ and $\left.\omega_{1}+2 \omega_{2}\right)$ will be removed, leaving the fundamental components of the two tones $\left(\omega_{1}\right.$ and $\left.\omega_{2}\right)$ and lower TOI products $\left(2 \omega_{1}-\omega_{2}\right.$ and $\left.\omega_{1}-2 \omega_{2}\right)$.

$$
\begin{aligned}
v_{\text {out }}= & \left(k_{1} B+\frac{9}{4} k_{3} B^{3}\right) \cdot\left[\cos \omega_{1} t+\cos \omega_{2} t\right] \\
& +\frac{3}{4} k_{3} B^{3} \cdot\left[\cos \left(2 \omega_{1}-\omega_{2}\right) t+\cos \left(\omega_{1}-2 \omega_{2}\right) t\right]
\end{aligned}
$$

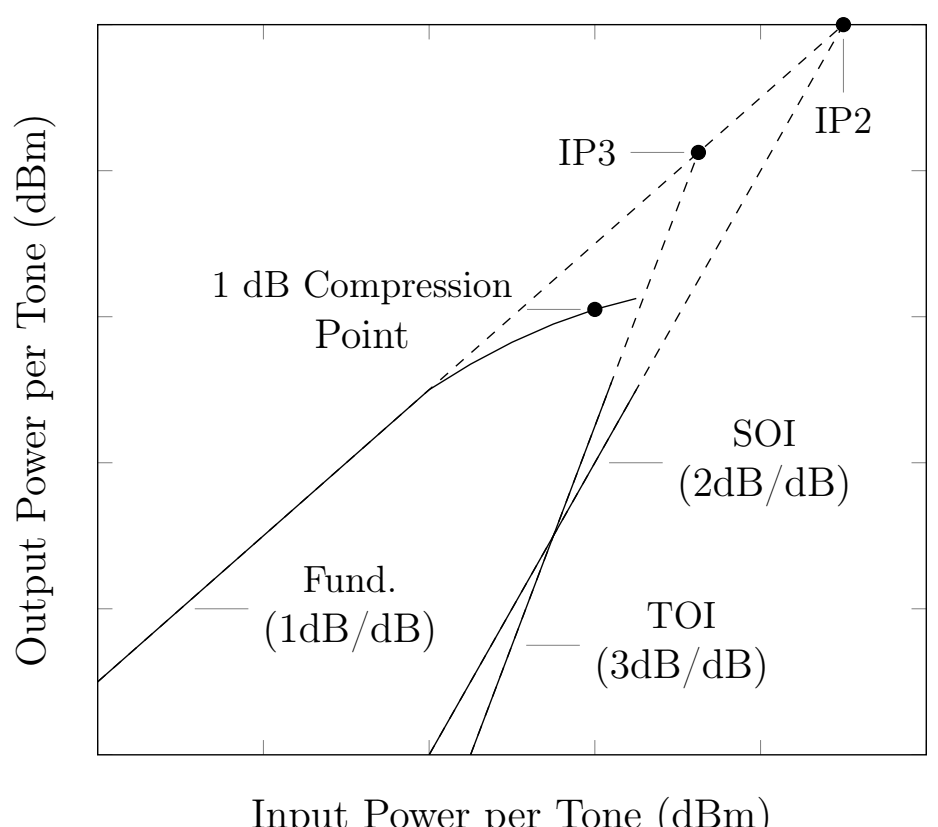

Figure 2.10: Nonlinear amplifier fundamental and intermodulated components showing slopes and intercept points. 


\section{Efficiency}

Fundamentally, an amplifier is a device that converts DC power to RF power and due to the high output power of a PA, high DC power is required to keep the device biased and conducting. Some of this DC power is dissipated as heat. The portion of the DC power converted to RF is known as the drain efficiency $\eta$.

$$
\eta=\frac{P_{o u t}}{P_{D C}}
$$

While a good indicator of overall efficiency (2.22) does not take into the input drive

level, which can quite high in PAs that often posses low gain. For this reason the PAE is most commonly used parameter for comparing PA performance.

$$
P A E=\frac{P_{\text {out }}-P_{\text {in }}}{P_{D C}}=\eta\left(1-\frac{1}{G}\right)
$$

\subsubsection{Classes of Operation}

Amplifiers are categorized into classes depending on the biasing, topology, and loadline characteristics. Amplifiers cannot be simultaneously designed for maximum linearity, bandwidth, efficiency, and power, and the various amplifier classes will either focus on maximizing a subset of these parameters at the expense of the others, or strike a compromise between them. The number of classes and topologies of amplifiers are almost as numerous and unique as the applications for which they are used. 
This section will provide an overview of the most common amplifier classes. The conduction angle of an amplifier is the portion of a full period for which the active device is conducting current. Classes $\mathrm{A}, \mathrm{B}, \mathrm{AB}$, and $\mathrm{C}$ are conduction angle mode amplifiers and they differ in the value of conduction angle and the effects this has on their linearity, efficiency, and output power. The FET input and output waveforms are shown in Figure 2.11. The output current goes to zero when the gate voltage drops below the threshold of the device, giving the waveform the resemblance of the piecewise function expressed below.

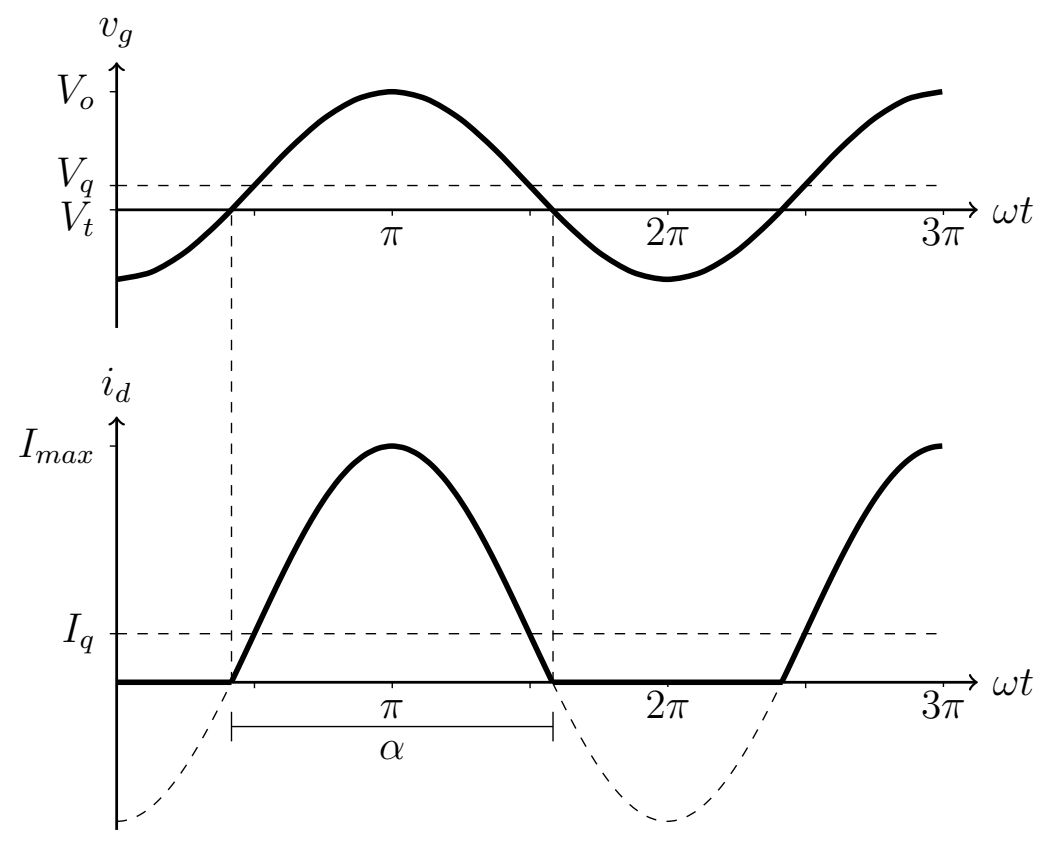

Figure 2.11: Reduced conduction angle gate voltage and drain current waveforms.

$$
i_{d}(\theta)=\left\{\begin{array}{l}
I_{q}+I_{p k} \cdot \cos \theta,-\alpha / 2<\theta<\alpha / 2 \\
0,-\pi<\theta<-\alpha / 2 ; \alpha / 2<\theta<\pi
\end{array}\right.
$$




\section{Class A}

An amplifier in class A operation is always conducting, giving it a conduction angle $(\alpha)$ of $360^{\circ}$. This allows any input signal variations to be immediately amplified and seen at the output, giving it the most accurate signal reproduction and greatest linearity of the amplifier classes. All small-signal amplifier are biased for class A operation but in the strictest sense a class A amplifier is not necessarily a linear or conjugate matched amplifier and may be operated into saturation or near the $1 \mathrm{~dB}$ compression point to serve as a PA. Since the amplifier is constantly carrying current even in the absence of an RF input signal, there is a continuous loss of power resulting in a theoretical maximum drain efficiency of $50 \%$. To achieve class A operation the FET must have a gate bias to ensure that the input voltage swing does not drop gate voltage past the threshold voltage of the device. This biasing, known as the quiescent (Q) point, is at the mid-point of the maximum device current in class $\mathrm{A}$ amplifiers (Figure 2.12). For most classes of amplifier, the drain is biased midway between the knee voltage and maximum voltage of the device, which is usually lower than the breakdown voltage of the device to provide a margin of safety and due to nonlinearities near the breakdown region.

\section{Class B}

To increase efficiency the dissipated power must be lowered; hence, either current must be minimal when voltage is at a maximum or vice-versa. One method of accomplishing 


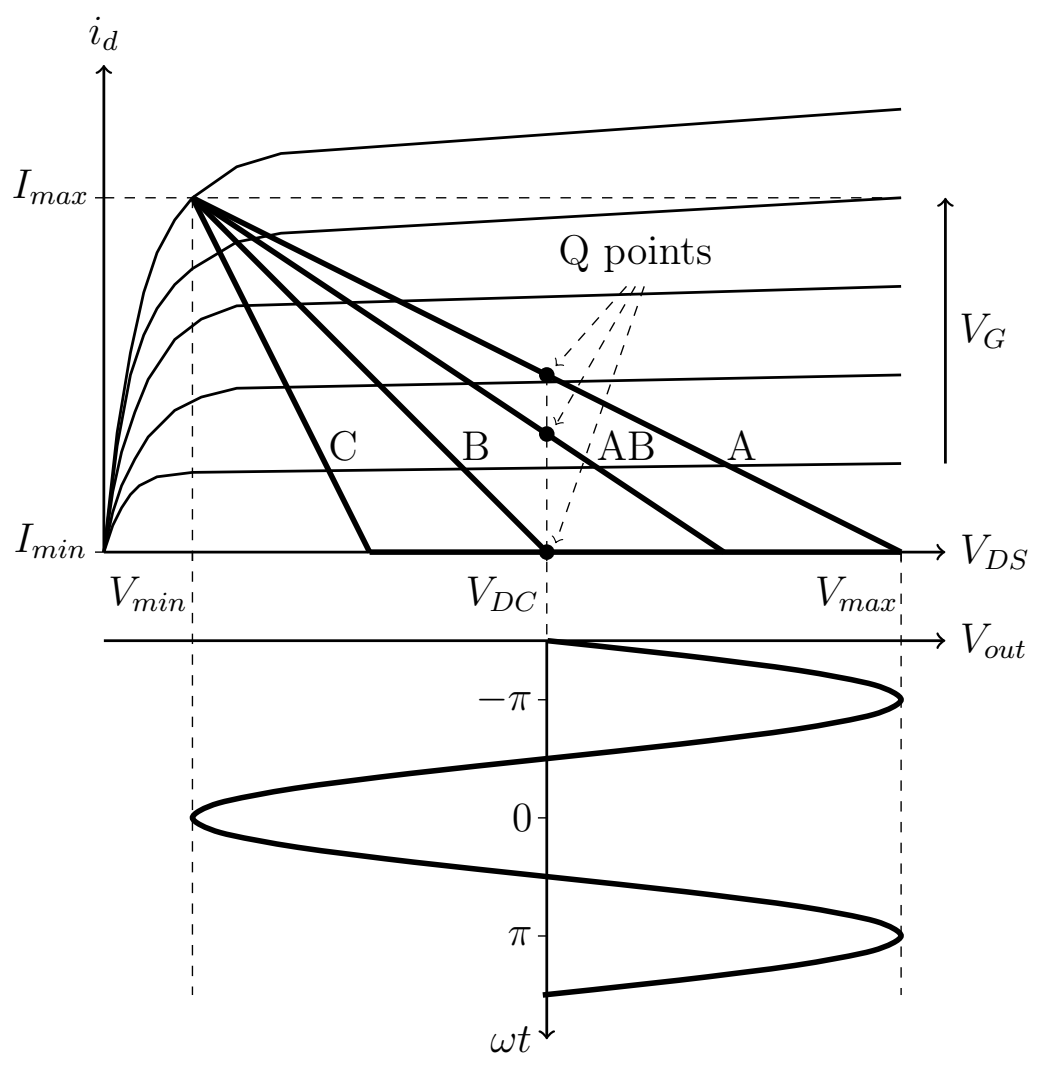

Figure 2.12: I-V curves of various amplifier classes.

this is to bias the device near the cutoff voltage so that the input voltage swing causes the device to turn off for a portion of the cycle resulting in no current flow across the device. This is the principle behind class $\mathrm{B}, \mathrm{AB}$, and $\mathrm{C}$ amplifiers. Class $\mathrm{B}$ amplifiers are biased at the threshold gate voltage resulting in no output current for the negative half of the cycle, giving them a conduction angle of $180^{\circ}$. In a single ended topology this results in a heavily distorted output waveform as only the positive half of the cycle is transfered to the output, and a maximum drain efficiency of $58 \%$, which is only a modest increase over class A. Therefore, class B amplifiers are most often implemented in a push-pull configuration that uses two complementary devices (n- 
type and p-type) with each conducting for one half-cycle. The devices' output signals combine to reconstruct the full cycle. Crossover distortion can be significant due to the non-zero threshold voltage of the FETs but this problem can be mostly alleviated by pre-biasing the devices using diodes. A theoretical maximum drain efficiency of $78.5 \%$ is possible using the push-pull configuration.

\section{Class AB}

Class AB amplifiers represent a middle ground between class A and B operation, with a conduction angle between $180^{\circ}$ and $360^{\circ}$ and corresponding maximum efficiency between $50 \%$ and $78.5 \%$. The Class AB amplifier offers a good compromise between linearity and efficiency in a single-ended approach which is often preferred to a pushpull configuration that requires transformers or complementary transistors that can be difficult to implement.

\section{Class C}

Class C amplifiers conduct for less than half the cycle (or a conduction angle between $0^{\circ}$ and $180^{\circ}$ ) and have a theoretical maximum drain efficiency of up to $100 \%$; however, at highly reduced conduction angles the output power drops significantly and the device's usefulness as an amplifier becomes questionable. The high degree of nonlinearity also makes this mode undesirable in many modulation schemes.

Table 2.3 summarizes the aforementioned amplifier classes' conduction angle and 
Table 2.3: Comparison of Amplifier Classes

\begin{tabular}{ccc}
\hline \hline Amplifier Class & Conduction Angle & Drain Efficiency (\%) \\
\hline A & $2 \pi$ & 50 \\
AB & $\pi-2 \pi$ & $50-78.5$ \\
B & $\pi$ & 78.5 \\
C & $0-\pi$ & $78.5-100$ \\
\hline
\end{tabular}

efficiency characteristics. It is possible to extract the output current components as a function of the conduction angle. These are given by (2.25) and (2.26) and plotted in Figure 2.13.

$$
\begin{gathered}
I_{D C}=\frac{1}{2 \pi} \int_{-\alpha / 2}^{+\alpha / 2} \frac{I_{\max }}{1-\cos (\alpha / 2)} \cdot(\cos \theta-\cos (\alpha / 2)) \cdot \mathrm{d} \theta \\
I_{n}=\frac{1}{\pi} \int_{-\alpha / 2}^{+\alpha / 2} \frac{I_{\max }}{1-\cos (\alpha / 2)} \cdot(\cos \theta-\cos (\alpha / 2)) \cdot \cos (n \theta) \cdot \mathrm{d} \theta
\end{gathered}
$$

\section{Class D}

Class D amplifiers are often fed a pulse-width modulated (PWM) binary signal that is a result of a comparator operation on a high frequency sawtooth waveform and the RF input signal. This effectively encodes the RF input signal into the low frequency portion of the PWM spectrum. After amplification the high frequency content is filtered out with a low pass filter. The amplifier is implemented in a push-pull configuration with complementary transistor pairs in a half or full-bridge topology. When 


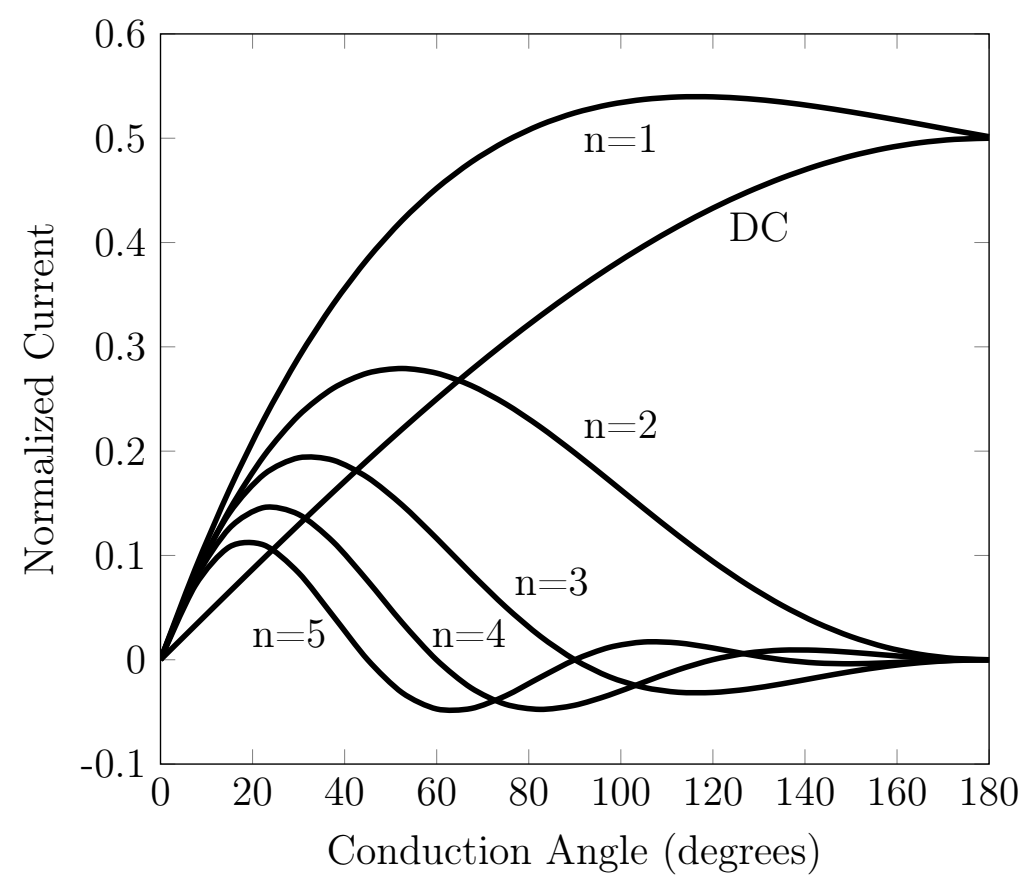

Figure 2.13: Current components as a function of conduction angle.

the input voltage is high the upper transistor conducts while the lower transistor is turned off and the positive supply voltage is fed directly to the output. When the input signal is low the lower transistor conducts while the upper transistor is turned off and the negative supply voltage (or ground) is fed directly to the output. For low on-resistance and high off-impedance transistors, these amplifiers can achieve a theoretical drain efficiency of $100 \%$. For sufficiently fast comparators and complementary transistors, class D amplifiers are superior to Class C when the goal is high efficiency and high output power. However, the difficulty of implementing high frequency comparators and the speed difference between complementary transistor pairs make the class D amplifier impractical at microwave frequencies. 


\section{Class E}

Class E amplifiers have an RF choke at the drain that stores energy while the transistor is on and the energy is delivered to the load when the transistor turns off. A filtering network at the load is used to shape the current and voltage waveforms such that they do not overlap and consume power allowing the class E amplifier to achieve a theoretical drain efficiency of up to $100 \%$. Drawbacks of class E operation includes a non-flat gain response, low output power and small bandwidth compared with traditional conduction angle mode amplifiers.

\section{Class F}

Class F amplifiers make clever use of harmonics to increase efficiency. They work by providing a high termination impedance at the second and third harmonics to increase the corresponding harmonic signals, creating a nearly rectangular output voltage waveform with a small transition time and hence low power loss. This allows them to achieve a theoretical drain efficiency of up to $90 \%$ or more with the addition of more harmonics.

\section{Other Classes}

Class $\mathrm{G}$ and $\mathrm{H}$ amplifiers use variable voltage rails to increase efficiency above class AB designs. In a class $\mathrm{G}$ amplifier the DC supply follows the output or input signal and switches between discreet voltage levels as the signal rises and drops. Less DC 
power is wasted in this manner because the difference between DC and output voltages are minimized. Class $\mathrm{H}$ amplifiers use a continuously variable DC supply to achieve even higher efficiency with the same method. The efficiencies obtained with class G and $\mathrm{H}$ amplifiers are higher than class $\mathrm{AB} / \mathrm{B}$ but lower than class $\mathrm{D}$.

\subsection{Planar Waveguide Transmission Lines}

At microwave and millimeter wave frequencies transmission lines are extensively used for realizing reactive elements and to provide well modeled interconnects. Discrete reactive components become more difficult to implement at these frequencies due to the relatively high parasitics and the increasingly small capacitance and inductance values needed to create matching networks and resonator structures. Figure 2.14 shows a length of transmission line and its distributed lumped element equivalent model. As the reactance is a function of length, transmission lines are scalable to allow realization of precise reactance values and only limited in value by allowable area. Another advantage of using transmission lines is that the ground return path helps to reduce magnetic and electric field coupling to nearby structures.

Applying Kirchhoff's voltage and current laws to the lumped element transmission line model and taking the limit as $\Delta z \rightarrow 0$, one can derive the telegrapher equations 


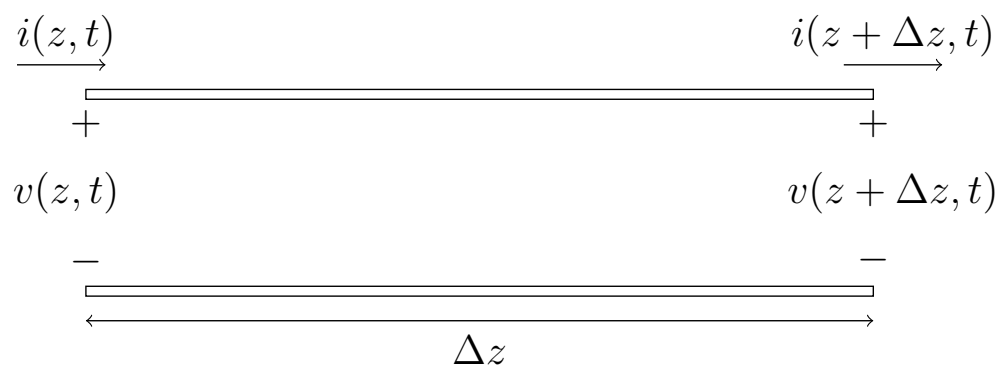

(a) Transmission line.

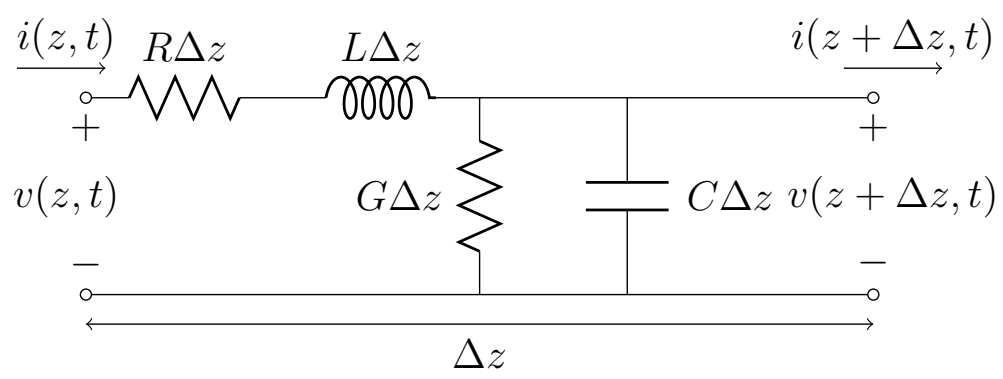

(b) Lumped element model.

Figure 2.14: Transmission line of length $\Delta z$.

[17].

$$
\begin{aligned}
& \frac{\partial v(z, t)}{\partial z}=-R i(z, t)-L \frac{\partial i(z, t)}{\partial t} \\
& \frac{\partial i(z, t)}{\partial z}=-G v(z, t)-C \frac{\partial v(z, t)}{\partial t}
\end{aligned}
$$

These equations can be solved for a steady-state condition and assuming a sinusoidal traveling wave to find the characteristic impedance of the transmission line as a function of the lumped element parameters.

$$
Z_{0}=\sqrt{\frac{R+j \omega L}{G+j \omega C}}
$$




\subsubsection{Microstrip}

Transmission lines can be realized in a planar topology with photolithographic processes in a multitude of ways. The earliest such form was stripline invented by Robert M. Barrett in the early 1950s while working for the Air Force Cambridge Research Center. Microstrip was invented shortly after by engineers of International Telephone \& Telegraph as a competitor to stripline [19]. These and other similar structures, shown in figure 2.15, are all considered types of microstrip waveguides. The first shown is the stripline, which has a conductor of width $w$ and thickness $t$ embedded within a dielectric substrate of permittivity $\epsilon_{r}$ and thickness $h$, and is sandwiched by two ground planes. It is not extensively used in modern day ICs except in special applications such as high Q filters despite its dispersion-less transverse electromagnetic (TEM) fundamental mode because active elements are difficult to realize in this type of embedded structure. Next is the conventional microstrip line, which has a conductor sitting atop a substrate with a ground plane on the back side. Its fundamental mode has small longitudinal electric and magnetic field components giving it a quasi-TEM mode (figure 2.16). It is also dispersive and the dispersion increases with frequency as its electric and magnetic fields become more concentrated within the substrate causing the effective dielectric constant to increase and its phase velocity to decrease. This is followed by the suspended microstrip line, which is similar to the conventional type but with an added air layer between the substrate and back ground plane. This allows the use of thinner substrates giving it lower dielectric losses and 
dispersion but it is more difficult to realize and hence, like the stripline, mostly sees use in filter applications The last structure is the coupled microstrip line, formed by two microstrip lines separated by a distance $s$, which can support both even and odd quasi-TEM modes.

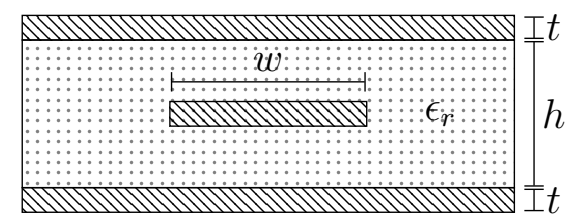

(a) Stripline.

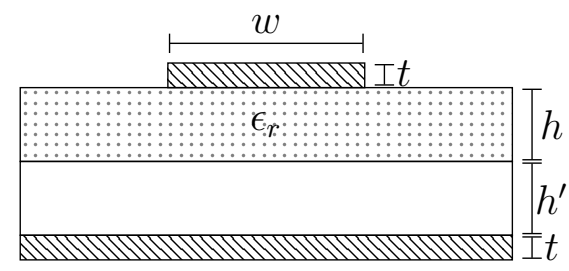

(c) Suspended microstrip line.

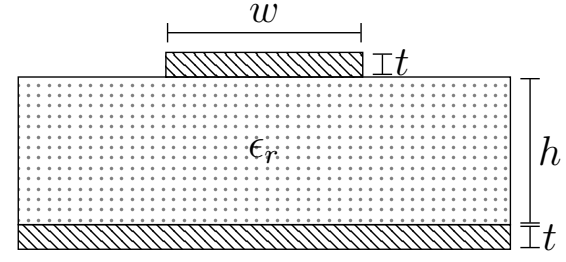

(b) Conventional microstrip line.

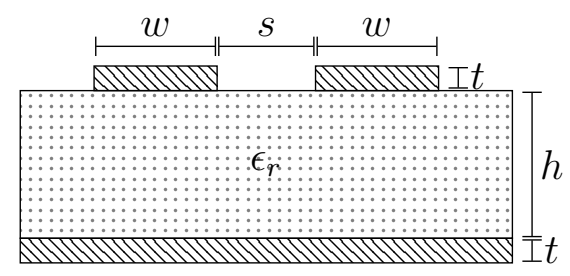

(d) Coupled microstrip lines.

Figure 2.15: Microstrip waveguides.

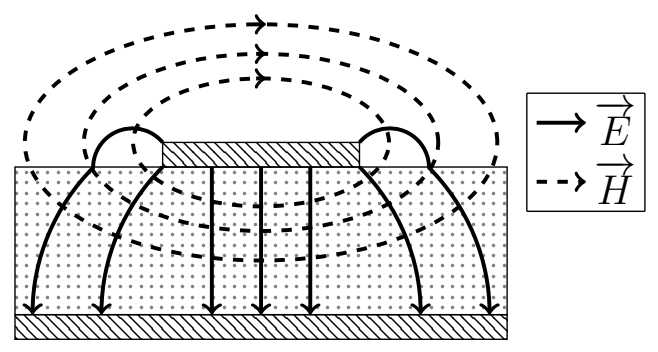

Figure 2.16: Electric and magnetic field distributions of a microstrip line. 


\subsubsection{Coplanar Waveguides}

Coplanar waveguides (CPWs) were invented in 1969 by Cheng P. Wen [20]. CPWs are characterized by the planar positioning of the center conductor strip and ground plane atop the dielectric substrate (or even and odd mode conductors in the case

of coplanar strips), although this does not preclude conductor backed waveguides or other structures in other planes within the substrate(s). The most common types are shown in figure 2.17. The first of these types is the coplanar strips with two parallel lines of width $w$ and separation $s$. It is similar to the coupled microstrip lines but without a conductor backing. This type of structure is rarely used at high frequencies due to the lack of a common ground plane leading to large stray fields but is sometimes used at lower frequencies in circuits with hybrid or lumped planar elements. The next structure is the conventional coplanar waveguide that has a center conductor of width $w$ and is flanked by a ground plane on either side separated by a distance $s$. This is the structure used in the design of this work and has many advantages over the other structures discussed here with few disadvantages that will be covered further below. The third structure is similar with a conductor backing but exhibits very different properties than the conventional CPW. When the separation is large and the width of the center to substrate height ratio is relatively large the field distribution is mostly concentrated within the substrate resulting in an increase in the effective dielectric constant and line attenuation, and has properties similar to that of the conventional microstrip line. As the separation decreases the 
coupling between the center conductor and adjacent ground planes increases and the structure exhibits properties closer to a conventional CPW. The last structure seen is the dielectric-material-backed coplanar waveguide which has a second substrate of different permittivity below the first. The properties of this structure are heavily dependent on the permittivities and heights of the two substrates but is otherwise similar to the conventional $\mathrm{CPW}$. In calculations a single layer reduction technique is used to combine these substrates (and the air/free space dielectric above the structure) into a single substrate with an effective permittivity and effective dielectric loss [21] [22].

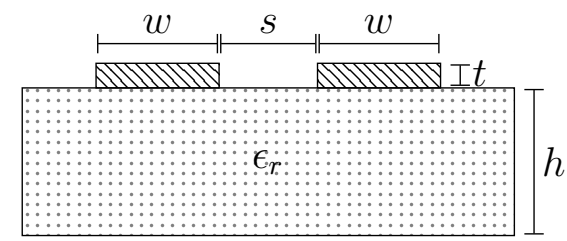

(a) Coplanar strips.

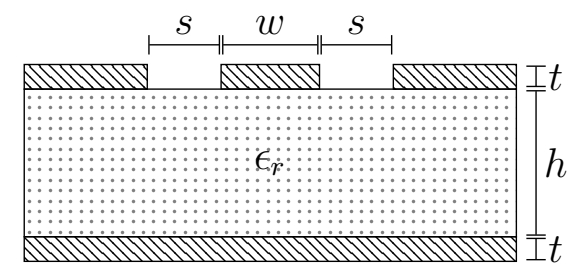

(c) Conductor-backed coplanar waveguide.

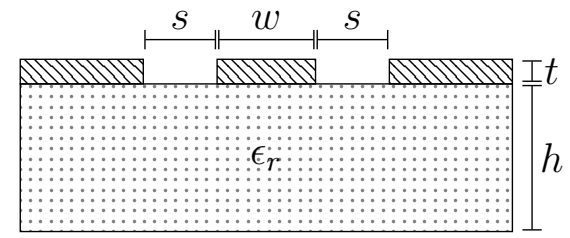

(b) Conventional coplanar waveguide.

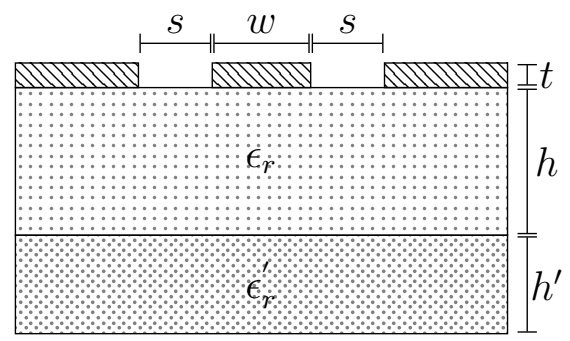

(d) Dielectric-materialbacked coplanar waveguide.

Figure 2.17: Coplanar waveguides.

CPW waveguides have a number of advantages over microstrip lines. The additional degree of freedom in design provided by the separation $s$ between center 


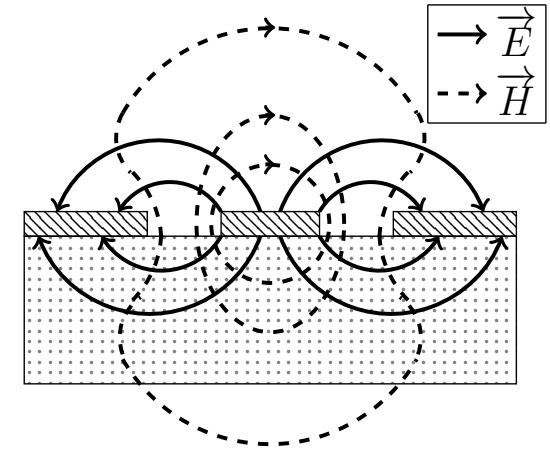

(a) Even mode.

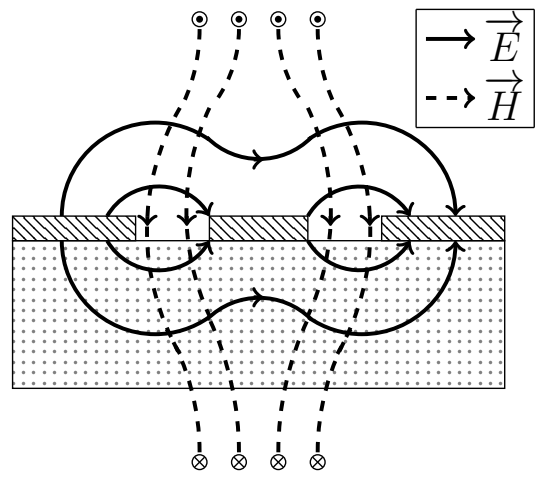

(b) Odd mode.

Figure 2.18: Electric and magnetic field distributions of the CPW even and odd modes.

conductor and adjacent ground plane gives the CPW a greater realizable impedance range, about 30-140 $\Omega$ compared with $25-95 \Omega$ for microstrip, and allows the use of thinner lines thereby reducing overall circuit area. There is also less cross-coupling between adjacent CPW lines compared with microstrip due to the intermediate ground plane, which allows for greater lines density and further reduces circuit area. The CPW is a truly planar circuit and doesn't require via holes to connect to a ground plane and makes for good ground contacts as well providing easier on-wafer measurements. This also allows for easy implementation of series and parallel active and passive elements. Without a conductor backing, there is no need to prepare the backside and thin the substrate as with microstrip. Therefore, CPWs can work with arbitrarily thick substrates which increases yield, especially when via holes are not used. The parasitic capacitance of the microstrip to backside ground parallel plates is non-existant, giving inductors and capacitors a higher self-resonant frequency (SRF). This allows the use of lumped elements such as spiral inductors and parallel plate ca- 
pacitors up into the $40-60 \mathrm{GHz}$ range. Finally, the $\mathrm{CPW}$ fundamental even mode is less dispersive than microstrip fundamental mode which allows for broadband circuits up to the millimeter wave to be used. [21].

In standard complementary metal-oxide-semiconductor (CMOS) processes the ground plane in microstrip is situated between the oxide and substrate layers with no electric field penetration into the substrate making the shunt loss dependent on the dielectric loss of the oxide only, giving it a capacitative quality (Q) factor of about 30. However, the proximity of the ground plane to the top signal trace gives it a very small distributed inductance and; therefore, low inductive Q-factor. In comparison, CPW lines can have either a large inductive Q-factor with high impedance or large capacitative Q-factor with low impedance due to the additional degree of freedom in design [23]. The main disadvantage of using CPW lines is that they are more difficult to design. In real application the ground plane is not only finite but varies in different points on the line and is likely asymmetric. This makes it difficult to model and do a priori design. EM simulators are required to give accurate results which increases computation time and design turn around [21].

CPWs can carry two fundamental modes with zero cutoff frequency, as seen in figure 2.18. These are the even or CPW mode with ground planes at the same potential, and the odd or slotline mode, with ground planes at potentials of equal magnitude but opposite sign. The even mode is quasi-TEM and is desirable due to its low dispersion allowing for broadband applications. The odd mode has significant 
dispersion due to a large longitudinal magnetic field component. In order to suppress the odd mode the two ground planes need to be kept at the same potential. This is achieved by the use of airbridges connecting one ground plane to the other placed along the CPW especially at discontinuities such as T-sections, bends, and discrete component connections.

\subsection{Programmable Tuners}

One method to achieve impedance tuning is to load a transmission line with voltage controlled elements such as varactors or switch-capacitors. The goal of programmable tuner is to provide a broad range of impedance values for maximum coverage. Therefore, the performance of varactors and switch-capacitors as programmable tuners depends on the maximum and minimum achievable capacitance that in turn define the minimum and maximum impedances, respectively. The switch capacitor topology has been shown to achieve a greater capacitance ratio, larger bandwidth, and higher Q-factor compared with varactors in a similar technology [24]. In addition, varactors are not available in the NRC GaN500 v2 process and would require an offchip solution. Therefore, a switch-capacitor topology (Figure 2.19) is chosen for this thesis.

When the gate voltage biases the FET into cut-off, a capacitance $C_{D S}$ develops across the drain-source junction and is in series with the fixed loading capacitor $C_{L}$ resulting in a capacitance minimum, and when the gate voltage biases the FET into 


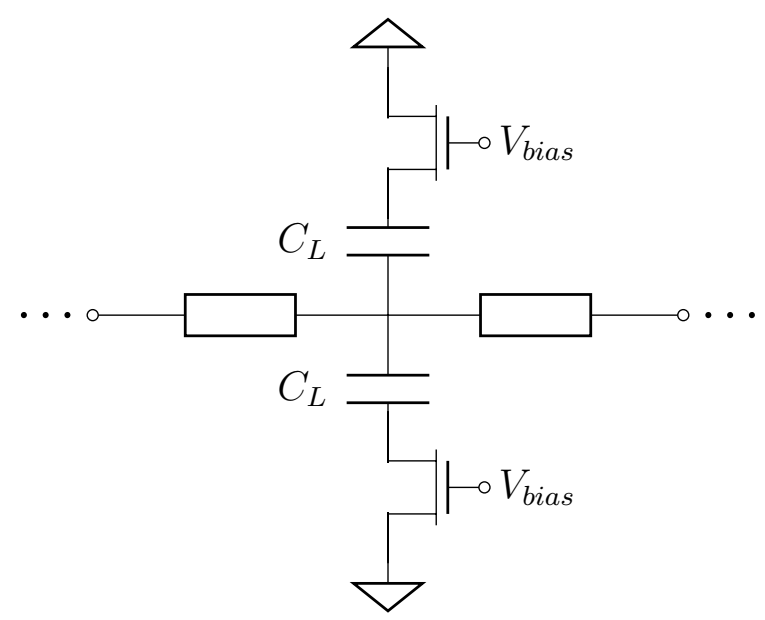

Figure 2.19: Switch-capacitor unit cell.

conduction the drain-source is effectively a short-circuit, resulting in a capacitance maximum.

$$
\begin{aligned}
C_{\text {min }} & =\frac{C_{L} C_{D S}}{C_{L}+C_{D S}} \\
C_{\text {max }} & =C_{L}
\end{aligned}
$$

The impedance tuning range is determined by the capacitance ratio:

$$
\frac{C_{\max }}{C_{\min }}=1+\frac{C_{L}}{C_{D S}}
$$

Figure 2.20 shows a loaded transmission line modeled using lumped elements. Assuming that losses on the line are negligible $(R=G=0)$ and using equation 2.28 one can derive the minimum and maximum impedance values of the unit cell based 
on this model.

$$
\begin{aligned}
& Z_{\text {min }}=\sqrt{\frac{s L}{C_{\max }+s C}} \\
& Z_{\text {max }}=\sqrt{\frac{s L}{C_{\text {min }}+s C}}
\end{aligned}
$$

where $\Delta z$ is the total length of the two halves of the transmission line, $L$ is the inductance per unit length, and $C$ is the capacitance per unit length. Apart from the impedance ratio, the insertion loss and linearity are important parameters to consider when designing the tuner. The Q-factor of the FET switches is the limiting factor in the insertion loss as the fixed capacitors have very high Q-factors.

$$
\begin{aligned}
Q_{C_{\text {min }}} & \approx \frac{1}{C_{D S} \omega R_{s}} \\
Q_{C_{\max }} & =\frac{1}{C_{L} \omega R_{O N}}
\end{aligned}
$$

where $R_{s}$ is the FET parasitic resistance, $R_{O N}$ is the FET series resistance in the ON state, and $C_{\text {min }} \approx C_{D S}$ where $C_{L} \gg C_{D S}$ has been assumed. A number of observations can be made from the above equations. Increasing the size of the fixed capacitor $C_{L}$ will increase the capacitance ratio but will also increase insertion loss. Using a wide FET width will lower the on-state resistance $R_{O N}$ but will increase the off-state capacitance $C_{D S}$ resulting in a lower capacitance ratio.

A single switch-capacitor will provide the lowest insertion loss for a given capacitance ratio but will only be able to switch between two impedance values, preventing 


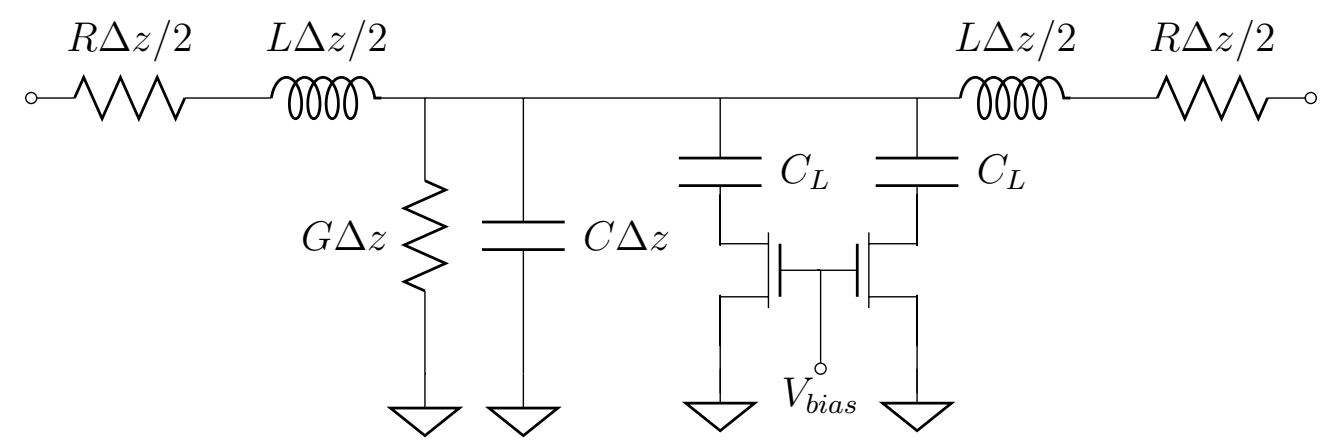

Figure 2.20: Equivalent lumped element model of switch-capacitor loaded transmission line.

any fine tuning of the impedance. Therefore, the switch-capacitor unit cell is cascaded $N$ times to create an $N$-bit tuner with $2^{N}$ possible impedance values.

\subsection{Antennas}

By solving Maxwell's equations it is observed that a time-varying current is necessary to create a self-sustaining EM field which will induce a similar current and voltage in the receiving antenna but may also couple into nearby objects, reducing the radiation power and efficiency, and modifying the antenna impedance and radiation pattern. To create a time-varying current there must either be discontinuities in the form of bends, steps, terminations or truncations, or a time-varying current amplitude.

Antennas may be separated into traveling-wave and standing-wave (or resonant) antennas. Traveling-wave antennas are either slow-wave or fast-wave (or leaky-wave) antennas depending on whether the phase velocity is slower or faster than the speed of light. Slow-wave antennas radiate at discontinuities at the beginning and end of the structure, in contrast to the fast-wave antennas that radiate continuously along 
their length. Compared with standing-wave antennas, traveling-wave antennas offer better bandwidth but lower directivity. Standing-wave antennas are carefully sized and matched to produce standing waves along their length at a particular wavelength. Since the antenna is only resonant at a single frequency (and its harmonics) standingwave antennas tend to be very narrowband.

The principle of operation for standing-wave antennas is easiest to understand with a monopole - a wire antenna one quarter wavelength long sitting above a ground plane. Charge flows into the input and reaches the end where it begins to build up and oppose further current flow. At this end current is at a minimum and in quadrature with the current at the input where it is maximum. Corollary, the charge buildup will cause a voltage maximum at the end and voltage minimum at the input. Therefore, impedance at the frequency of interest at the input will be minimal due to a combination of low current and high voltage.

\subsubsection{Antenna Types}

There are many antenna form factors and designs. Wire, aperture, reflector, and microstrip antennas make up the main categories. Each have a multitude of design options at a particular frequency depending on the required beam pattern and bandwidth. Wire antennas are seen in monopole, dipole, loop, and helical configurations and are simple and practical up to the low gigahertz frequencies. Aperture antennas are metallic waveguides with cut outs in the form of slots or open ends, which may 
then flare out in the shape of a horn. These antennas are most commonly used at microwave frequencies given their size, and come in many shapes; rectangular, circular, elliptical etc. Microstrip antennas consist of a metallic patch, often made of copper, resting on top of a dielectric substrate with a ground plane backing. This configuration makes them very easy to manufacturer with photolithographic processes, giving them a low profile and allowing them to be used in integrated circuits [25]. They can be fed by microstrip, probe, or aperture feeds. This type of antenna will be used in this thesis due the simplicity, low cost, and small size at the frequency of interest. It is not without disadvantages; however. A low efficiency and maximum power, poor polarization purity and scan performance, spurious feed radiation, and very narrow frequency bandwidth are the largest issues facing microstrip antenna designs [25]. Their form factor; however, makes highly desirable and prolific.

Other common antenna types include dielectric resonators, reflectors, and lenses. At millimeter wave frequencies conductor losses can be very high leading to low radiation efficiencies in metallic antennas. Unlike the previous antennas discussed, dielectric resonator antennas do not have a metallic radiative element and are able to achieve very high radiation efficiencies limited mostly by the dielectric loss tangent. Similar to microstrip antennas, they can take on a variety of shapes and have similar feed mechanisms [26]. Reflector and lens antennas are able to collimate a beam fed by a separate radiative element, resulting in very high gain for long range communication. To achieve collimation, reflectors use one or more reflective surfaces often in parabolic 
shape with a central feed, while lens antennas use transparent surfaces in convex or concave shape with an index of refraction different from air.

\subsubsection{Antenna Detuning}

Microstrip antennas take on many forms including, circular, rectangular, triangular, dipole, and ring shapes. The rectangular patch is perhaps the most common and simplest to analyze and is shown in Figure 2.21 having a length $L$ and width $W$, and fed by a microstrip transmission line. The edges of the patch form rectangular slots with the ground plane through which fringing fields emanate. The fringing fields from the slots on the sides along the length are out of phase and cancel while the fields from the slots at the ends along the width are in phase. These two slots are where the patch radiates and they may be modeled by an equivalent admittance circuit model $Y_{s}=G_{s}+j B_{s}$ with slot conductance

$$
G_{s}=\frac{W}{120 \lambda_{0}}\left(1-\frac{1}{24}\left(k_{0} h\right)^{2}\right)
$$

and slot susceptance

$$
B_{s}=\frac{W}{120 \lambda_{0}}\left(1-0.636 \ln \left(k_{0} h\right)\right)
$$

where $k_{0}$ is the wave number and $h$ is the slot (or substrate) height. At the resonant frequency, the susceptances of the two slots will cancel leaving only the resistive term (2.35). The resistance is maximal at the input of the patch (and minimal at the 
center) and is not necessarily matched to the impedance of the feed line, normally $50 \Omega$. Therefore, the feed needs to be moved in by a distance $s$ to avoid reflections at the feed to patch junction.

$$
R_{\text {in }}(s)=\frac{1}{Y_{\text {in }}}=\frac{1}{2 G_{s}} \cos ^{2}\left(\frac{\pi}{L} s\right)
$$

The dominant mode supported by the patch is the $T M_{010}$ mode with a resonant

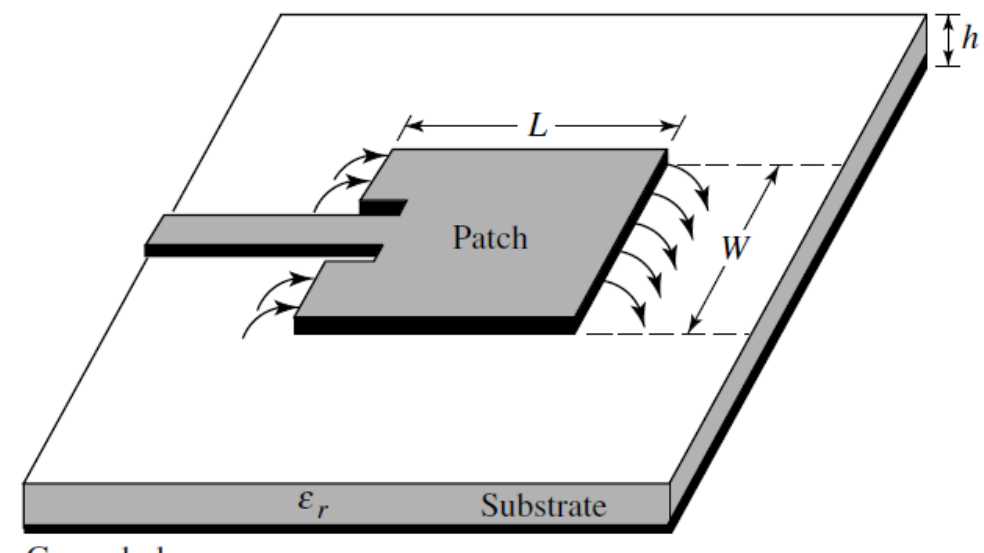

Ground plane

Figure 2.21: Rectangular microstrip antenna. [25]

frequency of approximately a half-wavelength.

$$
f_{r, 010}=\frac{c}{2 L_{e f f} \sqrt{\epsilon_{r_{e f f}}}}
$$

where $L_{\text {eff }}$ and $\epsilon_{\text {eff }}$ are the effective length and permittivity, respectively. Due to the fringing fields, the effective permittivity is not equal to the substrate permittivity but becomes a weighted average of the permittivity of the substrate and air. Empirical formulas exist for finding the effective permittivity and the resulting characteristic 
impedance for a typical microstrip line [27].

$$
\begin{aligned}
& \epsilon_{\text {reff }}=\left\{\begin{array}{l}
\frac{\epsilon_{r}+1}{2}+\frac{\epsilon_{r}-1}{2}\left[1+12 \frac{h}{W}\right]^{-1 / 2}, W / h>1 \\
\frac{\epsilon_{r}+1}{2}+\frac{\epsilon_{r}-1}{2}, W / h \leq 1
\end{array}\right. \\
& Z_{0}=\left\{\begin{array}{l}
\frac{120 \pi}{\sqrt{\epsilon_{e f f}}\left[\frac{W}{h}+1.393+0.667 \ln \left(\frac{W}{h}+1.444\right)\right]}, W / h>1 \\
\frac{60}{\sqrt{\epsilon_{\text {eff }}}} \ln \left(\frac{8 h}{W}+\frac{W}{4 h}\right), W / h \leq 1
\end{array}\right.
\end{aligned}
$$

The fringing fields will make the patch appear electrically longer than the physical length by an additional $2 \Delta L$ and wider by $2 \Delta W$, that is

$$
\begin{gathered}
L_{e f f}=L+2 \Delta L \\
W_{e f f}=W+2 \Delta W
\end{gathered}
$$

where

$$
\begin{aligned}
\Delta L & =0.412 \frac{\left(\epsilon_{\text {reff }}+0.3\right) \cdot\left(\frac{W}{h}+0.264\right)}{\left(\epsilon_{\text {reff }}-0.258\right) \cdot\left(\frac{W}{h}+0.8\right)} \\
\Delta W & =\frac{\ln 4}{\pi} h
\end{aligned}
$$

Combining equations (2.36), (2.39a), and (2.40a) and rearranging for the length gives

$$
L=\frac{\lambda_{0}}{2 \sqrt{\epsilon_{\text {reff }}}}-0.824 \frac{\left(\epsilon_{\text {reff }}+0.3\right) \cdot\left(\frac{W}{h}+0.264\right)}{\left(\epsilon_{\text {reff }}-0.258\right) \cdot\left(\frac{W}{h}+0.8\right)}
$$


To patch width is set to achieve a high radiation efficiency [25].

$$
W=\frac{\lambda_{0}}{2} \sqrt{\frac{2}{\epsilon_{r}+1}}
$$

\section{Detuning by Dielectric Materials}

This above analysis assumed that air or free space filled the volume above the antenna which is a reasonable approximation is some cases; however, if the antenna is situated outdoors precipitation can result in an added dielectric layer above the patch. As was seen in figure 2.16 the majority of the field lines in a microstrip line are confined to

the substrate; however, there are fringing field lines that exist in the region above the substrate, which is normally air.

The phase velocity of light in a medium is a function of the medium's permittivity.

$$
v_{p}=\frac{c}{\sqrt{\epsilon_{r} \mu_{r}}}
$$

where $c$ is the speed of light in free space and $\mu_{r}$ is the relative permeability and is very close to 1 for most materials, especially insulators. The relative permittivity is equal to 1 for air or free space, and is usually between 2.2 and 12 for the plastic or ceramic materials used in microstrip substrates. Substrates with lower permittivities and greater thickness are desirable for their better efficiency and larger bandwidth, but require larger area. Due to the fringing fields the relative permittivity of a microstrip transmission line is a combination of the permittivity of air and the substrate. This 
gives the microstrip an effective relative permittivity $\epsilon_{r e f f}$, which is an average perimittivity weighted by the proportion and strength of the field lines in each medium and can be expressed as the ratio of two capacitances.

$$
\epsilon_{\text {reff }}=\frac{C}{C_{0}}
$$

where $C$ is the capacitance per unit length of a TEM line in a filled dielectric and $C_{0}$ is that of a line in free space. For multi-dielectric layer substrates one can use the parallel plate capacitance with each layer connected in series to find an equivalent permittivity; however, the analysis for multiple dielectric layers above the microstrip is more involved. Nevertheless, an analytical expression was produced by [28] using a variational method to find the capacitances in (2.44).

$$
\frac{1}{C}=\frac{1}{\pi \epsilon_{0} Q^{2}} \int_{0}^{\infty} \frac{[f(\beta)]^{2}}{\left[F_{2}+\epsilon_{r, 1} \operatorname{coth}(\beta h)\right] \epsilon_{0} \beta h} d(\beta h)
$$

where $f(\beta)$ is the Fourier transform of the charge density distribution, whose trial function is given by

$$
f(x)=\left\{\begin{array}{l}
1+\left|\frac{2 x}{w}\right|^{3},-\frac{w}{2} \leq x \leq \frac{w}{2} \\
0, \text { otherwise }
\end{array}\right.
$$

and

$$
F_{i-1}=\epsilon_{r, i-1} \frac{\epsilon_{r, i-1} \tanh \left(\beta d_{i-1}\right)+F_{i}}{\epsilon_{r, i-1}+F_{i} \tanh \left(\beta d_{i-1}\right)}, i=n, n-1, \ldots, 3
$$


where $d_{i-1}$ is the thickness of the individual dielectric layers above the microstrip. From the above one can calculate the effective permittivity using (2.44), and combined with (2.38) find the change in impedance due to the insertion of additional layers of dielectric such as water or ice above the microstrip line. As the simulations performed in section ?? in this work and other studies have shown the resulting change in impedance can be significant enough to drastically impact performance.

\section{Detuning Due to Thermal Expansion}

In some locations the year round temperatures can vary as much as $105 \mathrm{~K}$ between summer and winter months [29]. The work in [30] analyzes the effect of temperature on antenna return loss, impedance, bandwidth, and center frequency. The physical size of the antenna was shown to change linearly with temperature by roughly $1.3 \% / \mathrm{K}$ corresponding to a shift in resonant frequency of $0.8 \% / \mathrm{K}$. The reason for the discrepancy is that the substrate also expands and the effective permittivity is reduced making the antenna electrically smaller, which helps to counter act the

effect of thermal expansion of the conductor. The impedance changed nonlinearly with the temperature, from the nominal value of $50 \Omega$ at $300 \mathrm{~K}$ to $40.74 \Omega$ at $296 \mathrm{~K}$ and $35.05 \Omega$ at $304 \mathrm{~K}$, a very drastic change for a modest temperature variation. Combined with the detuning due to ice and water layers, antennas exposed to the elements can suffer significant performance degradation without the use of a PIMN to compensate for the detuning. 


\subsection{Previous Works}

PIMNs have a multitude of use cases apart from the application in this work. Often times a multi-band solution is desired, especially in handsets, and PIMNs at the output, and often times also at the input, can be tuned for each frequency so that only a single PA is required. The majority of the previous works use a hybrid solution. Commercially available GaN HEMTs or InGaP/GaAs HBTs are often used with external fixed matching networks to provide a nominal match, then a microelectromechanical systems (MEMS) or PIN diode in series with a capacitor, inductor, or stub transmission line section is used to switch between two impedance states. These solutions require $N$ switches to provide $2^{N}$ impedance states. Varactors can be used to provide analog tuning; however, the small capacitance ratio limits their tuning range. This work uses a fully integrated PA and 3-bit PIMN using switch-capacitors in GaN and the simulated PA performance parameters and technologies used are compared with that of previous works in table 2.4 .

Table 2.4: Comparison with Previous Works

\begin{tabular}{|c|c|c|c|c|c|c|}
\hline Parameter & [31] & [32] & [33] & [34] & 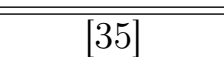 & $\overline{\text { This Work }}$ \\
\hline$P_{\text {sat }}(d B m)$ & 26 & - & 20 & - & - & 36 \\
\hline$P_{1 d B}(d B m)$ & 22 & 29 & - & 26 & 32 & 34 \\
\hline Gain $(d B)$ & 26 & 26 & - & 11 & 11 & 23 \\
\hline Area $\left(m m^{2}\right)$ & $1.5 \times 1.6$ & $5 \times 5$ & 31.3 & 10.5 & $6 \times 8$ & $4 \times 2$ \\
\hline Freq. $(G H z)$ & 5.5 & $0.8,1.95$ & $0.9,1.8$ & 1.84 & 2 & 10 \\
\hline Tuner Type & SWH-Cap & $\begin{array}{l}\text { MEMS } \\
\text { SWH-Cap }\end{array}$ & $\begin{array}{l}\text { MEMS FBK } \\
\text { SWH-Cap }\end{array}$ & $\begin{array}{l}\text { Tunable } \\
\text { T-Line }\end{array}$ & $\begin{array}{l}\text { BST MEMS } \\
\text { Varactor }\end{array}$ & SWH-Cap \\
\hline PA Process & $\begin{array}{l}90 \mathrm{~nm} \\
\mathrm{CMOS}\end{array}$ & $\begin{array}{l}\text { Std. HBT } \\
\text { InGaP/GaAs }\end{array}$ & $\begin{array}{l}0.35 \mu \mathrm{m} \\
\mathrm{CMOS}\end{array}$ & $\begin{array}{l}\text { Std. HBT } \\
\text { InGaP/GaAs }\end{array}$ & $\begin{array}{l}\text { Std. HEMT } \\
\text { GaN }\end{array}$ & $\begin{array}{l}500 n m \\
\mathrm{GaN}\end{array}$ \\
\hline
\end{tabular}


Numerous GaN PAs have been designed for X-band operation. A two-stage $\mathrm{PA} /$ rectifier is implemented in [36] with a $150 \mathrm{~nm} \mathrm{GaN}$ on $\mathrm{SiC}$ process. It achieves a $10 \mathrm{~W}$ saturated output power with $20 \mathrm{~dB}$ gain and PAE of $50 \%$ at $9.9 \mathrm{GHz}$. In [37] a $250 \mathrm{~nm}$ GaN transistor with a gate width of $4.8 \mathrm{~mm}$ was shown to output $25 \mathrm{~W}$ pulsed power with a gain of $12 \mathrm{~dB}$ and efficiency of $41 \%$ with a drain bias of $50 \mathrm{~V}$ in a $6 \mathrm{~mm} \times 6 \mathrm{~mm}$ package. Commercially, there are numerous companies developing GaN PAs at this frequency. The TriQuint TGA2624-CP has a saturated output power of $42 \mathrm{dBm}$ with a gain of $27 \mathrm{~dB}$, maximum PAE of $37 \%$ within a $15.2 \mathrm{~mm} \times 15.2 \mathrm{~mm} \times 3.5 \mathrm{~mm}$ package [38]. Hittite's line of GaN PAs include the single stage HMC1087F10, which has a saturated output power of $38.5 \mathrm{dBm}$, gain of $11 \mathrm{~dB}$ in a $11.4 \mathrm{~mm} \times 17.3 \mathrm{~mm}$ package [39], and the HMC7149 with a saturated output power of $40 \mathrm{dBm}$, gain of $20 \mathrm{~dB}$ in a $3.4 \mathrm{~mm} \times 4.5 \mathrm{~mm} \times 0.1 \mathrm{~mm}$ package [40]. 


\section{Design}

\subsection{Specifications}

The specifications outlined in section 1.4 are summarized in table 3.1. The specifications should consider the abilities of the process and provide goals to the designer that will allow for a safe design while attempting to maximum performance within the given constraints, especially the chip size which is rather limited for a fully integrated PA and PIMN design in a $500 \mathrm{~nm}$ process at $10 \mathrm{GHz}$. The measured results will be compared to the specifications to determine the effectiveness of the design methodology, simulations and models.

Table 3.1: Specifications

\begin{tabular}{cccc}
\hline \hline Parameter & Value & Units & Min/Nom/Max \\
\hline Output Power, $P_{1 d B}$ & 30 & $\mathrm{dBm}$ & Min \\
Gain, $G$ & 12 & $\mathrm{~dB}$ & Min \\
Return Loss, $S 11, S 22$ & 12 & $\mathrm{~dB}$ & Min \\
Efficiency, $P A E$ & 30 & $\%$ & Min \\
Center Frequency, $f_{0}$ & 10 & $\mathrm{GHz}$ & Nom \\
Bandwidth, $B W$ & 0.5 & $\mathrm{GHz}$ & Min \\
Area, $A$ & $4 \times 2$ & $\mathrm{~mm}^{2}$ & Max \\
\hline
\end{tabular}

\subsection{Power Amplifier Design}

\subsubsection{Biasing and Matching Networks}

Figure 3.1 shows a block diagram of the biasing and matching networks for a generic FET based amplifier. There are three common options for providing the gate and 
drain biasing to the active device as seen in figure 3.2. An RF choke provides a large impedance at the frequency of operation while passing DC current and needs to have a high Q-factor for a minimal voltage drop, and is realized using a large value inductor to provide an attenuation typically on the order of $20 \mathrm{~dB}$ or greater. In a practical design, a shunt capacitor would also be used to provide an $\mathrm{AC}$ ground at the bias pin. If the characteristic impedance of the RF line is $50 \Omega$ the inductor would need to have a reactance of $5000 \Omega$ or greater requiring an inductance of

$$
\begin{aligned}
Z_{L} & =j \omega L_{\text {choke }} \\
L_{\text {choke }} & \geq \frac{j 5000 \Omega}{j 2 \pi 10^{10} \mathrm{GHz}} \\
& \geq 80 \mathrm{nH}
\end{aligned}
$$

This inductance can be realized in the NRC GaN process using a spiral inductor (figure 2.3c) with 5.5 turns, occupying a $290 \mu \mathrm{m} \times 290 \mu \mathrm{m}$ area and using reinforced 2ME on $1 \mathrm{ME}$ to provide a higher Q-factor than a single metal layer topology. $1 \mathrm{ME}$ has a maximum current carrying capacity of $6 \mathrm{~mA} / \mu \mathrm{m}$, while $2 \mathrm{ME}$ on $1 \mathrm{ME}$ has a capacity of $12 \mathrm{~mA} / \mu \mathrm{m}$. At $1 \mathrm{~W}$ output power with $30 \%$ efficiency and a drain bias of $30 \mathrm{~V}$, the DC current is roughly $110 \mathrm{~mA}$ requiring a line width greater than $9 \mu \mathrm{m}$ for $1 \mathrm{ME}+2 \mathrm{ME}$ or $18 \mu \mathrm{m}$ for $1 \mathrm{ME}$ only. The saturation current is likely to be significantly higher, by a factor of two or greater; therefore, a minimum line width of $20 \mu \mathrm{m}$ for $1 \mathrm{ME}+2 \mathrm{ME}$ and $40 \mu \mathrm{m}$ for $1 \mathrm{ME}$ is used. The fabrication process also requires a minimum line spacing of $5 \mu \mathrm{m}$. However, using such a large and tightly wound 


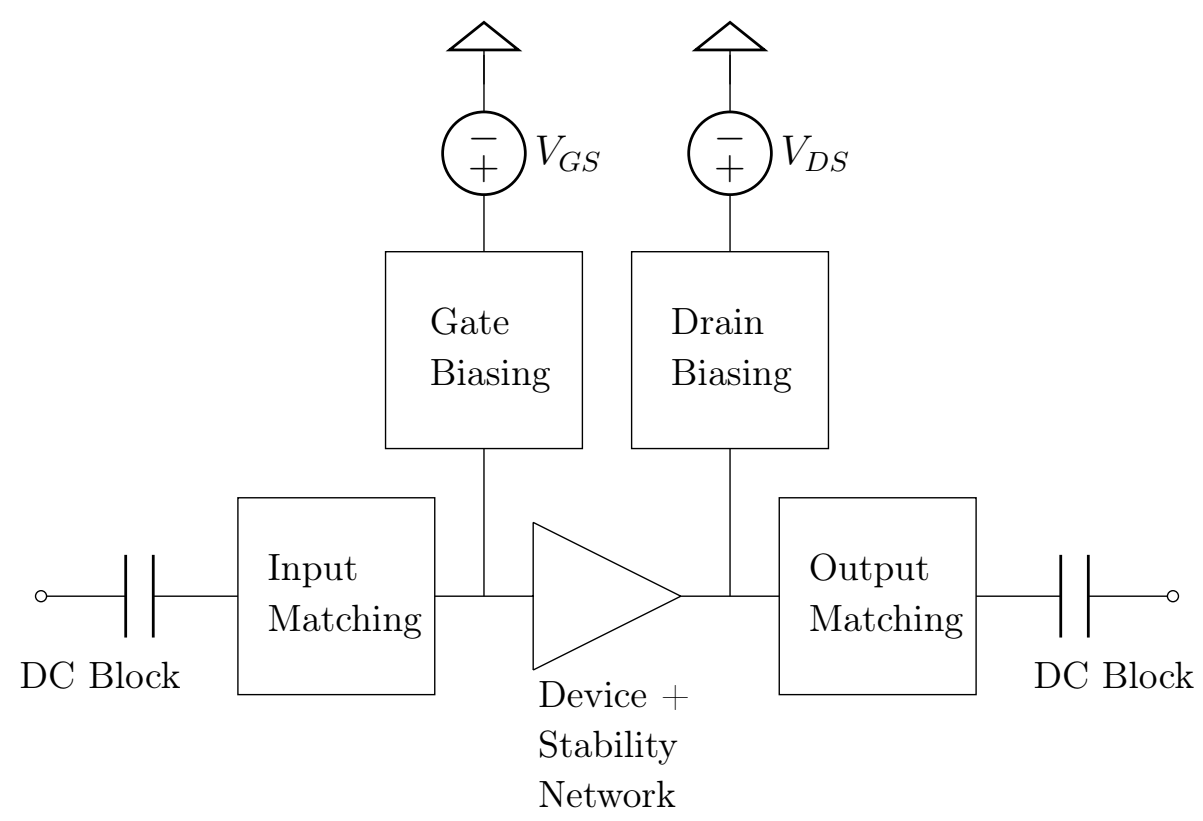

Figure 3.1: Amplifier biasing and matching networks.

inductor greatly increases the parasitic capacitance and places the $\mathrm{SRF}$ at $10.3 \mathrm{GHz}$ and very close to the operating band. The slope of the inductance as a function of frequency is much greater than zero resulting in a very narrow band solution. In addition the inductor occupies a large area and does not provide impedance matching to the device.

Another solution is the quarter-wave transmission line with an $\mathrm{AC}$ ground provided by a large capacitor that transforms the short circuit at RF line to an open 


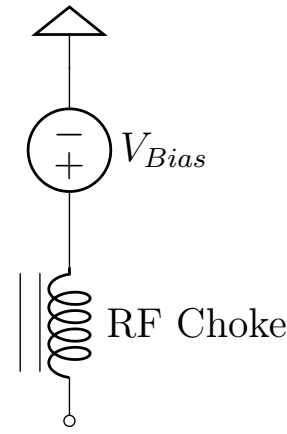

(a) $\mathrm{RF}$ choke.

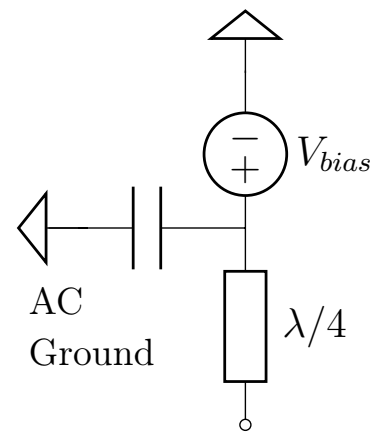

(b) Quarterwave transformer.

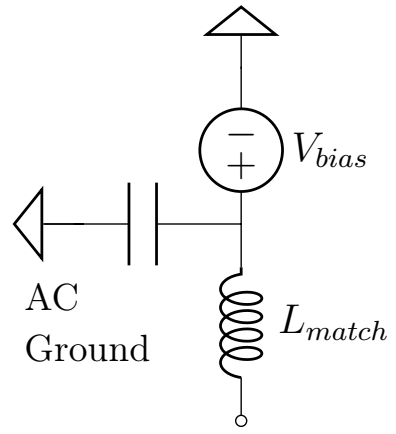

(c) Matching inductor.

Figure 3.2: Biasing network topologies.

circuit at the AC ground. The physical length of the quarter-wave line is given by

$$
\begin{aligned}
\lambda / 4 & =\frac{c}{4 f \sqrt{\epsilon_{\text {reff }}}} \\
& \approx \frac{3.0 x 10^{8} \mathrm{~m} / \mathrm{s}}{4 \cdot 10^{10} \mathrm{GHz} \sqrt{9.5}} \\
& =2.43 \mathrm{~mm}
\end{aligned}
$$

Even with the use of meandered lines, four such quarter-wave transmission lines for a two-stage PA would occupy a large amount of space on the chip. In addition, the quarter-wave line is very narrowband due to its frequency dependent length.

The third option uses a large AC coupling capacitor to provide an AC ground at the bias pin and a matching inductor and is the most compact option. The inductor values are easily realizable with low parastics and place the inductor far from its SRF and area is greatly reduced as the matching network is already partially realized for a 
two element LC match. However, not every impedance is realizable and the matching network is a high pass filter. Nevertheless, this topology is chosen in this work for its compactness.

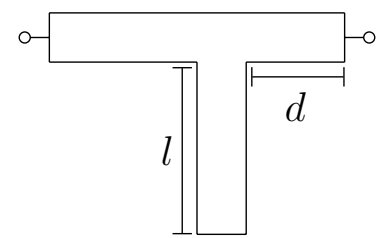

(a) Open circuited stub.

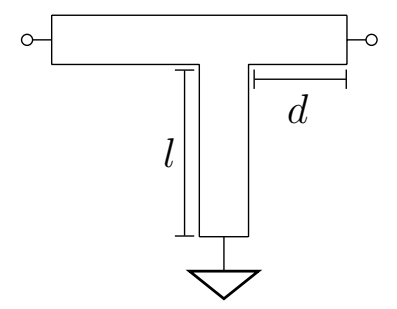

(b) Shortcircuited stub.

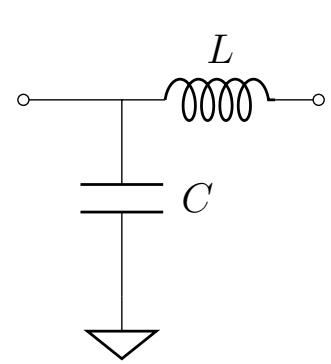

(c) Lowpass LC.

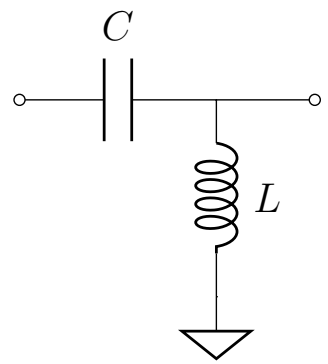

(d) Highpass LC.

Figure 3.3: LC and single stub matching network topologies.

Some of the possible matching network topologies are seen in figure 3.3 and include two-element LC networks and single stubs. A stub may have an open or short circuit termination and is placed a distance $d$ away from the load to which it is matched. The offset distance $d$ is chosen so that the conductance of the load is transformed to $Y_{0}=1 / Z_{0}=1 / 50 \Omega$. Then the length of the stub $l$ is sized to remove the remaining susceptance to provide a matched condition [17]. If the load admittance is $Y_{L}=1 / Z_{L}=G_{L}+j B_{L}$ then the solutions for the offset distance $d$ are

$$
\frac{d}{\lambda}=\left\{\begin{array}{l}
\frac{1}{2 \pi} \tan ^{-1} t, t \geq 1 \\
\frac{1}{2 \pi}\left(\pi+\tan ^{-1} t\right), t<1
\end{array}\right.
$$


where

$$
t=-\frac{B_{L} Z_{0}}{2}
$$

and the solutions for the length of the stub are

$$
\frac{l}{\lambda}=\left\{\begin{array}{l}
\frac{-1}{2 \pi} \tan ^{-1}\left(\frac{X}{Z_{0}}\right), \text { for short-circuited stubs } \\
\frac{1}{2 \pi} \tan ^{-1}\left(\frac{Z_{0}}{X}\right), \text { for open-circuited stubs }
\end{array}\right.
$$

where

$$
X=\frac{G_{L}^{2} t-\left(Y_{0}-t B_{L}\right)\left(B_{L}+t Y_{0}\right)}{Y_{0}\left[G_{L}^{2}+\left(B_{L}+Y_{0} t\right)^{2}\right]}
$$

Similar to the quarter-wave transmission line, the length of the stub matching networks will depend on the frequency of operation. To match to an impedance of, say, $(57+\mathrm{j} 86) \Omega$ would require an offset distance of $d=0.256 \lambda=2.49 \mathrm{~mm}$ and a stub length of $l=0.162 \lambda=1.57 \mathrm{~mm}$ for an open-circuited stub, or $l=0.412 \lambda=4.00 \mathrm{~mm}$ for a short-circuited stub. The limited area available makes the LC matching network more desirable. The high-pass network can also be used to provide biasing and the series capacitor has the added value of providing a DC isolation for the bias network.

\subsubsection{Power Amplifier Stage Design}

The maximum power density the device can support is $10 \mathrm{~W} / \mathrm{mm} \mathrm{DC}$ and in order to obtain an output power of $1 \mathrm{~W}$ RF the device needs to be sized appropriately. 
Combining (2.22) and (2.23) and solving for the output power gives

$$
P_{\text {out }}=\frac{P A E \cdot G \cdot P_{D C}}{(G-1)}
$$

Dividing both sides by the device area $A$ and re-arranging gives

$$
A=\frac{P_{\text {out }}(G-1)}{P A E \cdot G \cdot\left(P_{D C} / A\right)}
$$

Substituting $P_{D C} / A=10 \mathrm{~W} / \mathrm{mm}, P_{\text {out }}=1 \mathrm{~W}, P A E=0.3, G=12 \mathrm{~dB}=16$ gives a minimum required area of $A=313 \mu \mathrm{m}$. The GaN devices from the NRC can be fabricated with gate finger widths of $25 \mu \mathrm{m}, 50 \mu \mathrm{m}, 100 \mu \mathrm{m}$, or $200 \mu \mathrm{m}$ with either 2, 4 or 8 fingers. Therefore, the minimum size required to give a $1 W$ output is $400 \mu \mathrm{m}$ and can be achieved using a $2 \times 200 \mu \mathrm{m}, 4 \times 100 \mu \mathrm{m}$ or $8 \times 50 \mu \mathrm{m}$ device. Increasing the number of fingers will decrease the parasitic resistance but increase the capacitance. Increased parasitic resistance may be useful as both the $4 \times 100 \mu \mathrm{m}$ and $8 \times 50 \mu \mathrm{m}$ devices are unstable at $10 \mathrm{GHz}$ without the addition of stabilizing resistors or a feedback stability network. The input impedance of the device is lower for smaller gate finger widths, resulting in a high transformation ratio making the input matching network narrowband and, especially in the case of the 8 finger device, difficult to realize. In addition, the 8 finger device is very wide, requiring a very large taper to prevent significant reflections at the discontinuity between the transmission line and device. However; the $2 \times 200 \mu \mathrm{m}$ device is very long which would make it 
difficult to fit within the chip area alloted without meandering the RF line. For these reasons a $4 \times 100 \mu \mathrm{m}$ device was chosen as a compromise.

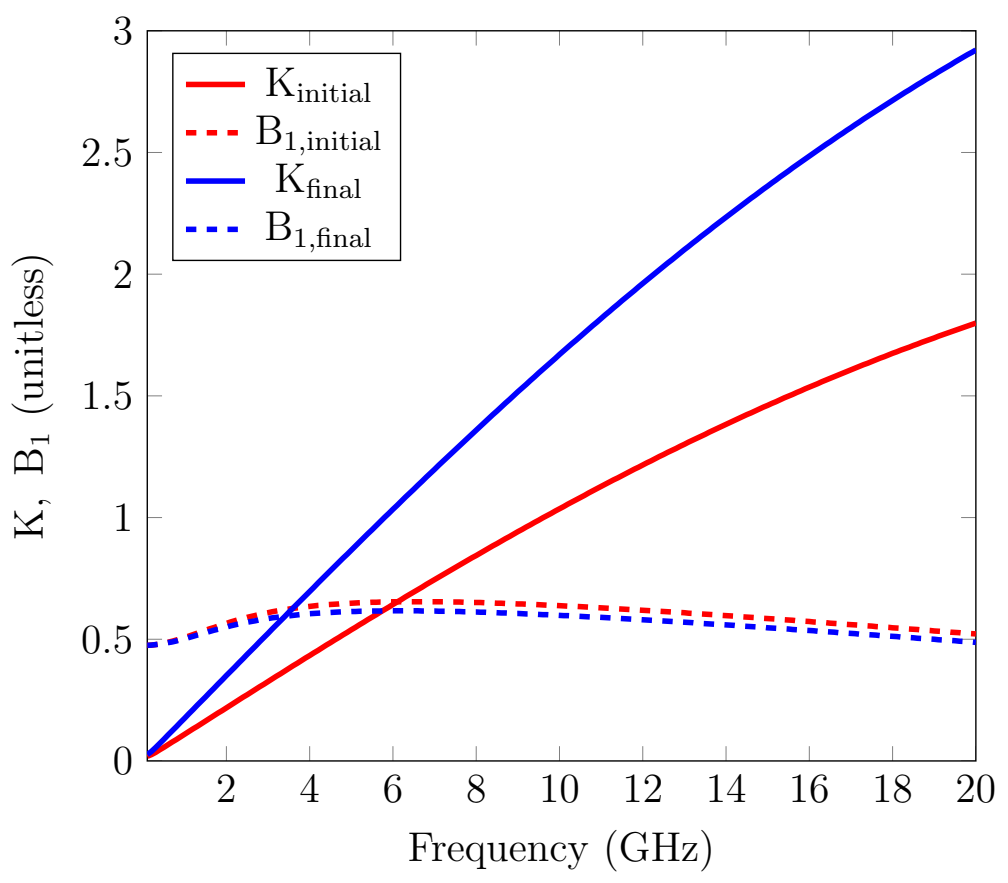

Figure 3.4: Stability factor and stability measure before and after addition of $2 \Omega$ series resistor.

Amplifier stability is an important consideration in design. Instability caused by feedback from the transistor output to input can cause spurious oscillations to occur. This is possible when the input or output ports have a negative real impedance or equivalently if either of the input and output reflection coefficients are greater than unity. The source and load matching network, or any external source or load impedances not matched to $50 \Omega$ will modify the reflection coefficients and as such may move the amplifier into a region of instability. Amplifiers that are susceptible to instability of this type are conditionally stable in that they are stable for a certain of range of passive source and load impedances. Amplifiers that are stable for all passive 
source and load impedances are unconditionally stable. Stability can be frequency dependant due to the matching networks' frequency dependent impedance [17]. One method of measuring amplifier stability is to calculate the Rollett stability factor $K$ and auxiliary stability factor $B_{1}$ from S-parameter measurements [18].

$$
\begin{gathered}
K=\frac{1-\left|S_{11}\right|^{2}-\left|S_{22}\right|^{2}+|\Delta|^{2}}{2\left|S_{12} S_{21}\right|} \\
B_{1}=1+\left|S_{11}\right|^{2}-\left|S_{22}\right|^{2}-|\Delta|^{2} \\
\Delta=S_{11} S_{22}-S_{12} S_{21}
\end{gathered}
$$

An amplifier is said to be unconditionally stable if $\mathrm{K}>1$ and $\mathrm{B}_{1}>0$. An S-parameter simulation was performed (figure 3.4) to calculate the stability metrics above. Initially, the device is potentially unstable in the band of interest. With the addition of a $2 \Omega$ series resistor at the input to the gate the device shows unconditional stability for frequencies above $6 \mathrm{GHz}$.

I-V curves of the device are seen in figure 3.5 for a common source configuration, which were obtained by sweeping the drain voltage $\left(V_{D S}\right)$ from 0 to $60 \mathrm{~V}$ for gate voltages $\left(V_{G S}\right)$ ranging from -5 to $+2 \mathrm{~V}$ in $1 \mathrm{~V}$ increments. The saturation current with no gate voltage applied was found to be $322 \mathrm{~mA}$ giving a current density of $0.803 \mathrm{~A} / \mathrm{mm}$, as was expected from the process parameters given in table 2.2. The 


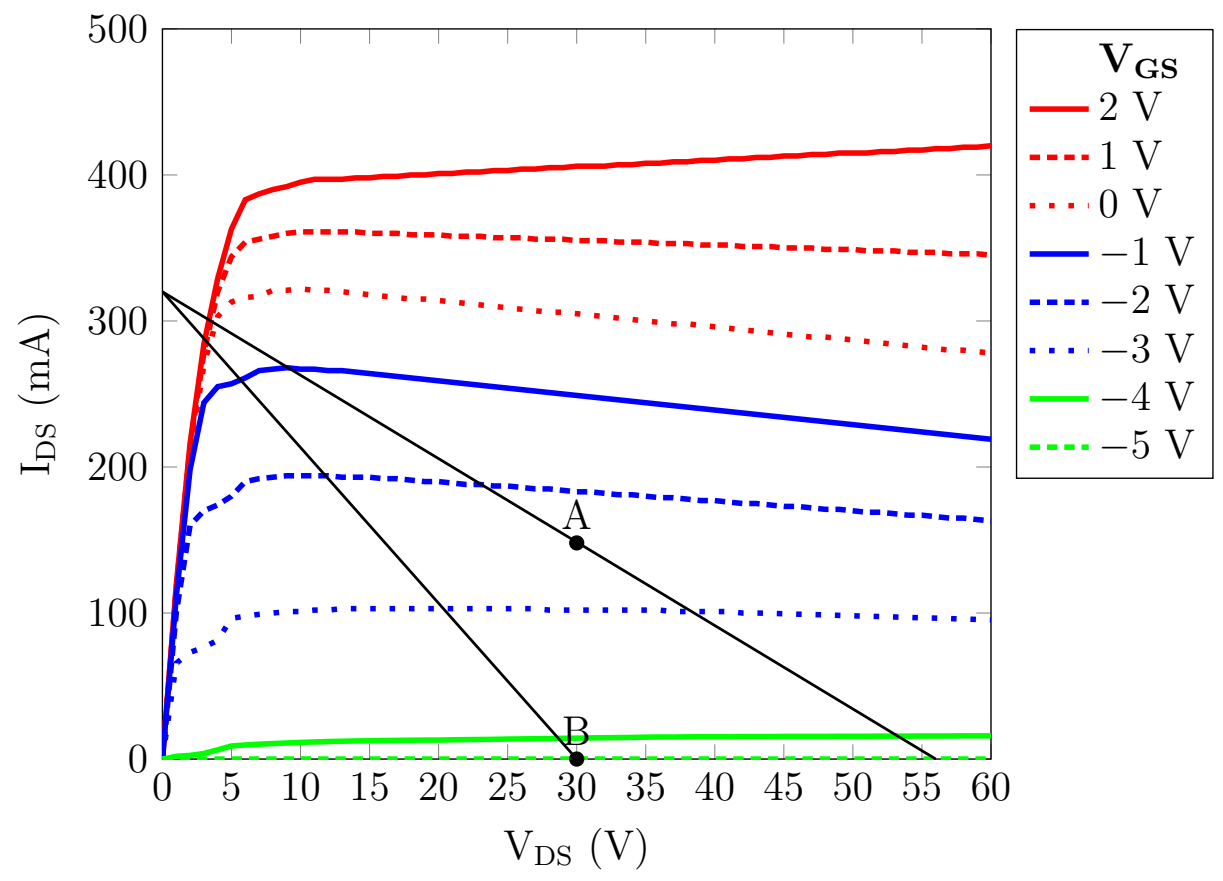

Figure 3.5: $4 \times 100 \mu \mathrm{m}$ FET I-V curves.

simulation model was found to be extrapolating values at voltages higher than $30 \mathrm{~V}$. In addition, the capacitor maximum operating voltage is $40 \mathrm{~V}$ [14] and a safety should be applied; therefore, the drain bias was set to $30 \mathrm{~V}$. The knee voltage is roughly $5 \mathrm{~V}$, allowing a positive voltage swing up to $55 \mathrm{~V}$ in class $\mathrm{A}$ operation. The FET will operate in class $\mathrm{AB}$ mode to ensure linear operation with increased efficiency compared with class A. Class A biasing occurs at a gate bias of $-2.3 \mathrm{~V}$ and the cutoff is at about $-4.2 \mathrm{~V}$ for class B. However, according to table 2.2 the threshold voltage can vary from $-3.6 \mathrm{~V}$ to $-4.6 \mathrm{~V}$ so in order to ensure correct biasing for class $\mathrm{AB}$ the gate bias should be between $-2.3 \mathrm{~V}$ and $-3.6 \mathrm{~V}$.

Using (2.10) where $I_{\min }=0 \mathrm{~mA}, I_{\max }=322 \mathrm{~mA}, V_{\min }=5 \mathrm{~V}, V_{\max }=55 \mathrm{~V}$, an initial estimate of $R_{o p t}=155 \Omega$ for the optimal resistance was obtained. Next, 


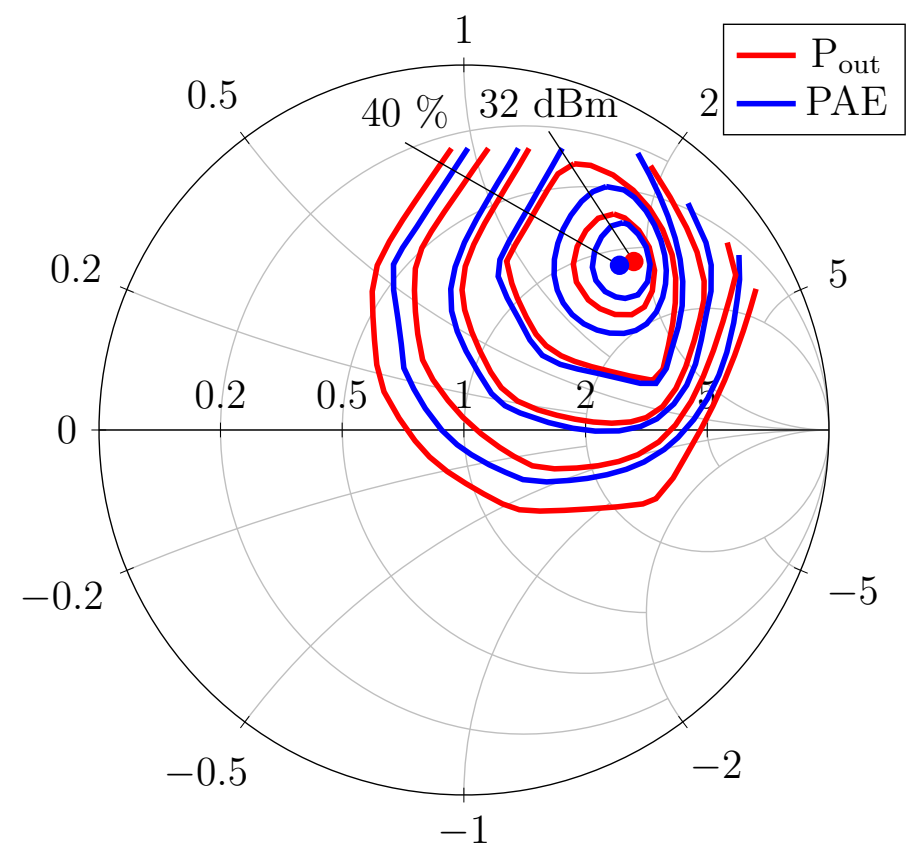

Figure 3.6: Load pull simulation of 4 x $100 \mu \mathrm{m}$ FET showing constant power delivered and PAE contours with $1 \mathrm{dBm}$ and $5 \%$ intervals, respectively.

a load pull simulation was carried out with complex impedances swept around this initial value while sweeping the input power level for $V_{G S}$ between -2.3 and $-3.6 \mathrm{~V}$ in $0.1 \mathrm{~V}$ increments. The results in figure 3.6 are for an input power of $22.5 \mathrm{dBm}$, with a gate bias of $-3.2 \mathrm{~V}$ and show constant output power and $P A E$ contours. The maximum points occur at roughly the same output impedance, approximately $(57+\mathrm{j} 86) \Omega$ at an input impedance of $(2-\mathrm{j} 12) \Omega$, corresponding to a PAE of $40 \%$ and output power of $32 \mathrm{dBm}$ for a gain of $9.5 \mathrm{~dB}$. High-pass LC matching networks are designed to match these impedances to $50 \Omega$ at the input and output as seen in figure 3.7 . 


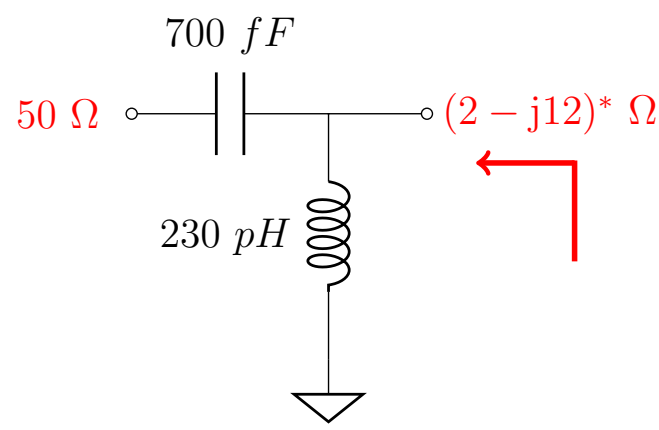

(a) Input matching network.
$190 f F$

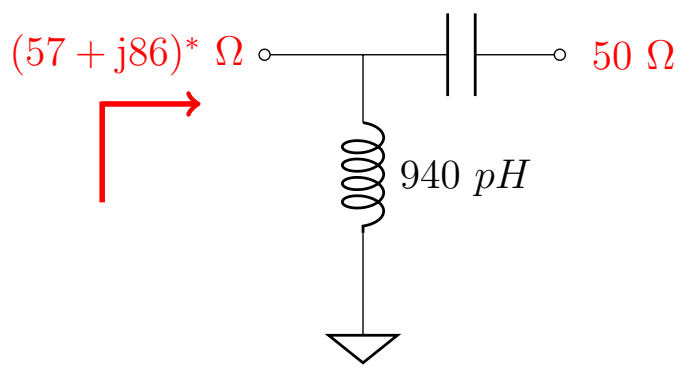

(b) Output matching network.

Figure 3.7: PA matching networks.

\subsubsection{Driver Stage Design}

In order to get sufficient gain a driver amplifier stage was designed as well. A $4 \times 50 \mu \mathrm{m}$ device is chosen to give a 2:1 gate width ratio between the second and first stage. As the devices were found to be bilateral $\left(S_{12} \neq 0\right)$ a simultaneous conjugate match was required and the solutions for the required reflection coefficients are given in (2.8). Since the device is unconditionally stable at $10 \mathrm{GHz}, K>1$ and $B_{1}>0$, which implies that $|\Delta|<1$ and; therefore, $\left|B_{1} / 2 C_{1}\right|>1$ and $\left|B_{2} / 2 C_{2}\right|>1$. Only one solution is possible in this case.

$$
\begin{aligned}
& \Gamma_{S}=\frac{B_{1}-\sqrt{B_{1}^{2}-4\left|C_{1}\right|^{2}}}{2 C_{1}} \\
& \Gamma_{L}=\frac{B_{2}-\sqrt{B_{2}^{2}-4\left|C_{2}\right|^{2}}}{2 C_{2}}
\end{aligned}
$$

The S-parameters obtained from simulation at $10 \mathrm{GHz}$ are $S_{11}=0.79 \angle-119^{\circ}$, 


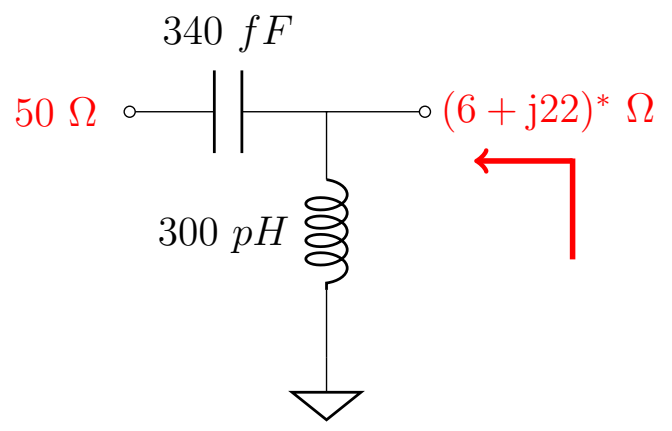

(a) Input matching network.

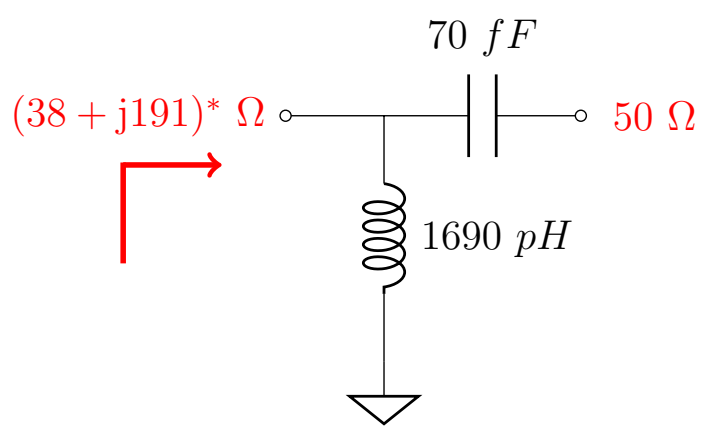

(b) Output matching network.

Figure 3.8: Driver matching networks.

$S_{22}=0.89 \angle-23^{\circ}, S_{12}=0.02 \angle 8^{\circ}$, and $S_{21}=1.95 \angle 97^{\circ}$. Using (2.9) and substituting into (3.12) gives $\Gamma_{S}=0.83 \angle 132^{\circ}$ and $\Gamma_{L}=0.91 \angle 28^{\circ}$ corresponding to impedances of $(6+j 22) \Omega$ and $(38+j 191) \Omega$, respectively. Once again these impedances are matched to $50 \Omega$ using high-pass LC matching networks (figure 3.8).

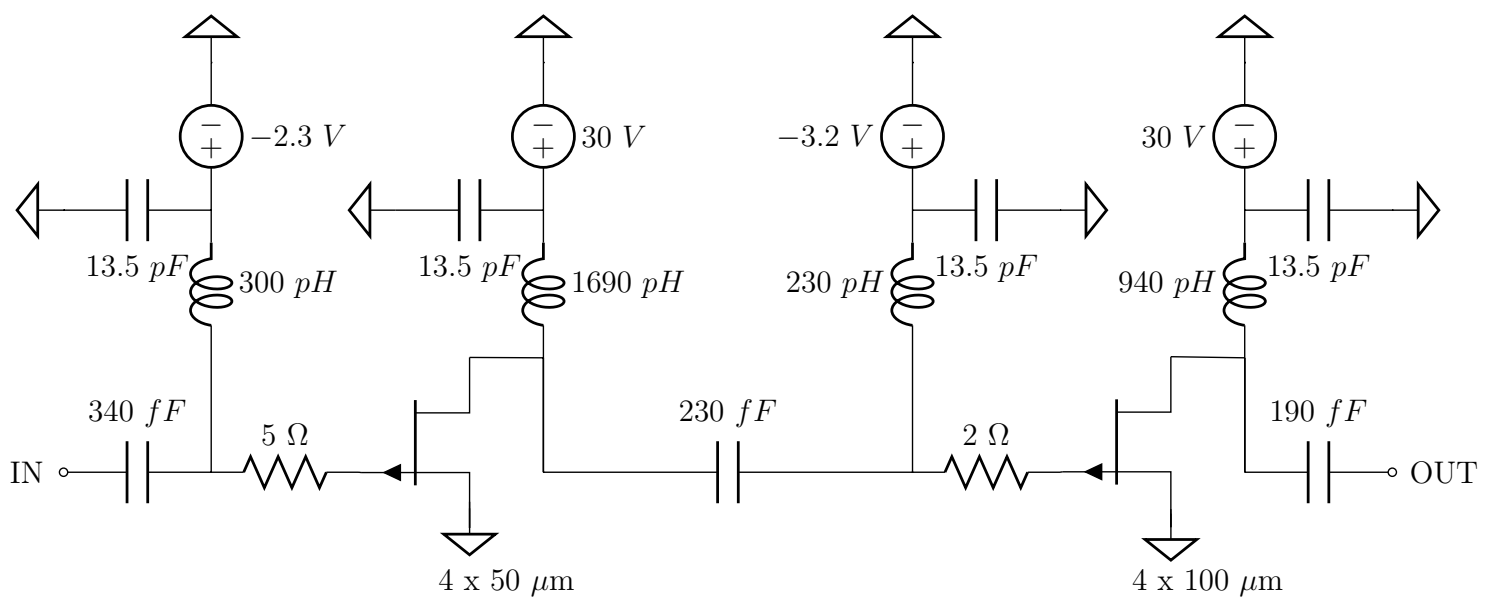

Figure 3.9: Two-stage PA schematic.

The two stages are then combined into a single circuit (figure 3.9). The inductors forming the matching networks are also used to bias the active devices along with a $13.5 \mathrm{pF}$ capacitor (the largest value allowed in the process) to provide an AC ground 
condition. The interstage match is simplified by combining the two series capacitors which also eliminates the need for a large DC blocking capacitor between the two stages.

\subsection{Antenna Detuning Simulations}

While the detuning effects of dielectric objects including water or ice buildup on antennas have been researched, most of the previous studies used exposed RFID tag dipoles or Yagi antennas near $1 \mathrm{GHz}$ [41] [42]. To get a more accurate view of the effects for the application in this work, an X-band microstrip patch antenna was designed and simulated using a finite element method (FEM) simulator in the Keysight EMPro environment (figure 3.10). Rain will cause the antenna to become wet and surface tension will prevent the water from uniformly spreading to single molecule thickness or rolling off the surface. The thickness of a puddle of liquid on a surface under surface tension is given in [43].

$$
t=\sqrt{\frac{2 \gamma(1-\cos \theta)}{g \rho}}
$$

where, $\gamma$ is the liquid's surface tension, $\rho$ is its density, $\theta$ is the contact angle of the surface, and $g=980 \mathrm{~cm} / \mathrm{s}^{2}$ is the acceleration due to gravity on the surface of the earth. The maximum thickness occurs for a contact angle of $180^{\circ}$ and in the case of water at $25^{\circ} \mathrm{C}, \gamma=72 \mathrm{dyn} / \mathrm{cm}$ and $\rho=1.0 \mathrm{~g} / \mathrm{cm}^{3}$, resulting in a thickness of 


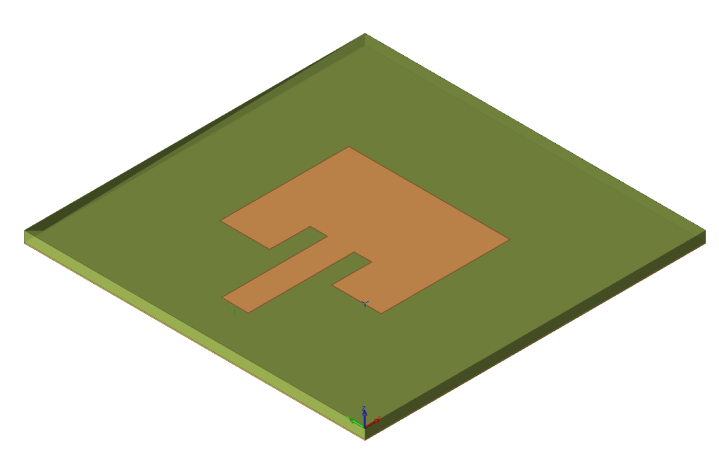

(a) Bare antenna.

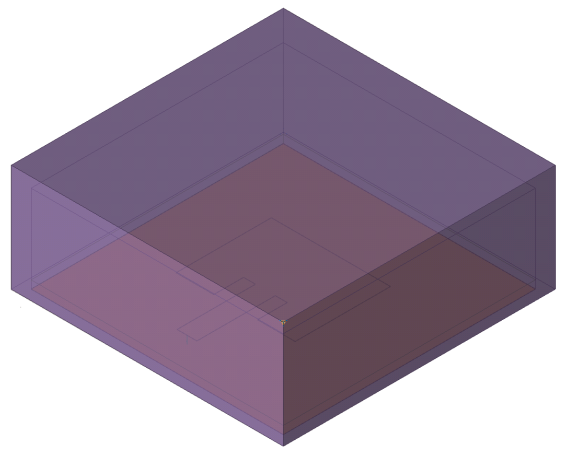

(b) Antenna with radome.

Figure 3.10: 3D models of simulated antennas.

Rogers RT/Duroid ${ }^{\circledR} 5880$ polytetrafluoroethylene (PTFE) composite laminate was chosen for this design due to its low loss dissipation factor at $10 \mathrm{GHz}(\tan \delta=$ 0.0009). It has a permittivity of $\epsilon_{\mathrm{r}}=2.2$ and comes in a standard thickness of $31 \mathrm{mil}$ or $0.787 \mathrm{~mm}$ with $1 \mathrm{oz}$. copper cladding spread over a $1 \mathrm{ft}^{2}$ area for a thickness of $35 \mu \mathrm{m}$ [44]. Using (2.42) the width of the patch was calculated to be $\mathrm{W}=11.9 \mathrm{~mm}$. Then using (2.37) the effective permittivity was calculated to be $\epsilon_{\text {reff }}=2.0$ and was used to calculate the length of the patch using (2.41) and was found to be $\mathrm{L}=9.7 \mathrm{~mm}$. Combining (2.33) and (2.35) the feed inset required to match to $50 \Omega$ was calculated to be $3.0 \mathrm{~mm}$. After simulating the antenna the dimensions were optimized to a $0.1 \mathrm{~mm}$ accuracy to get the correct center frequency and matching. Most practical microstrip antennas include a radome to shield them from the elements. A $10 \mathrm{~mm}$ high and $1 \mathrm{~mm}$ thick rectangular radome made of acrylonitrile butadiene styrene (ABS) plastic material was placed over the antenna. The radome has a detuning effect on the antenna so once again the dimensions were optimized to get a $50 \Omega$ 
match. Table 3.2 shows the antenna dimensions for the initial calculation and the optimized cases with and without a radome.

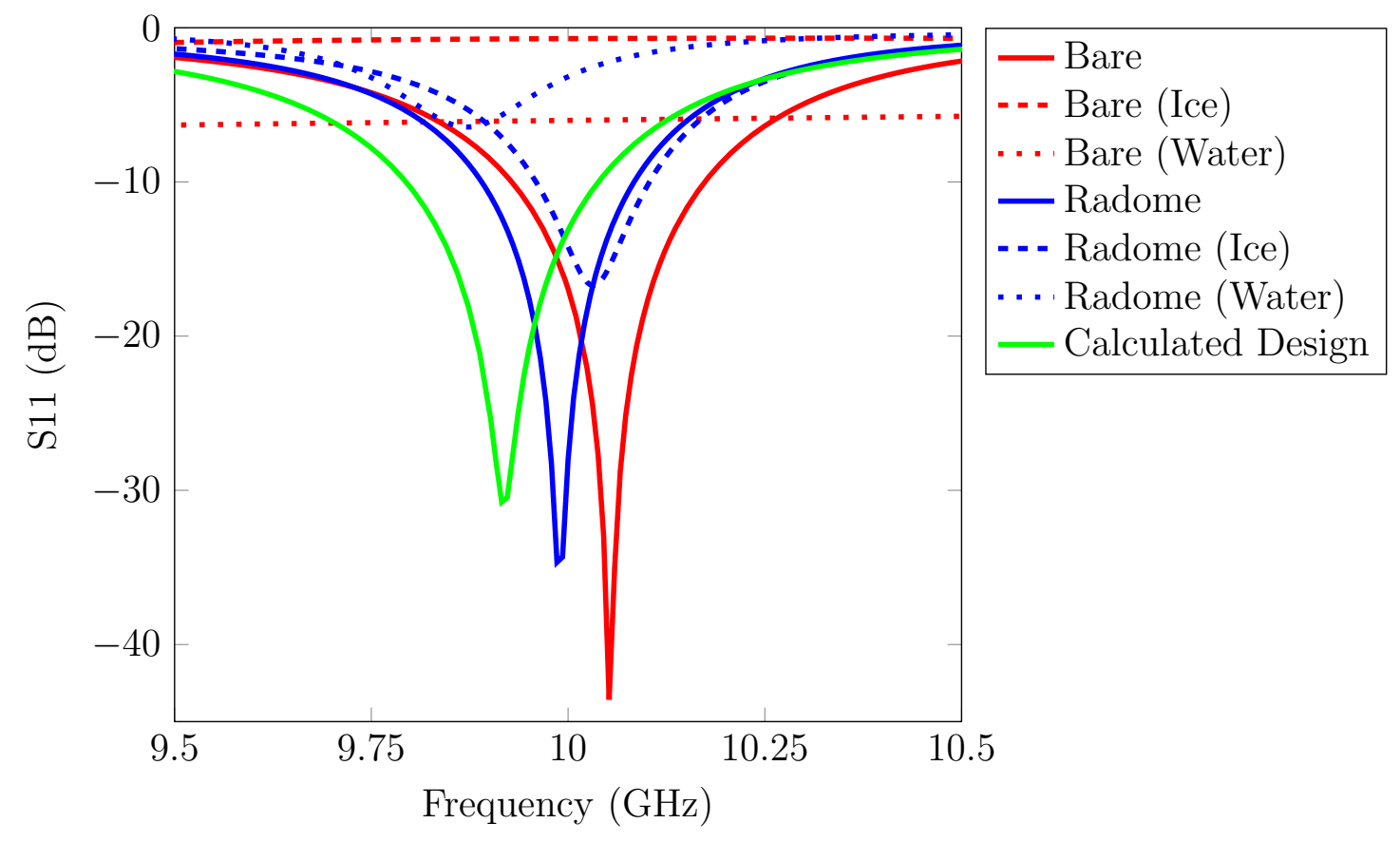

Figure 3.11: Antenna return loss.

Table 3.2: Initial and Final Antenna Dimensions in Millimeters

\begin{tabular}{cccccc}
\hline \hline Antenna & Length & Width & Feed Inset & Feed Width & Gap \\
\hline Calculated & 9.7 & 11.9 & 3.0 & 2.3 & 1 \\
Optimized for free space & 9.5 & 11.8 & 3.0 & 2.3 & 0.9 \\
Optimized for radome & 9.6 & 12.0 & 3.0 & 2.0 & 1.3 \\
\hline
\end{tabular}

Next both antennas were covered in a layer of water with various thicknesses ranging from 1 to $4.4 \mathrm{~mm}$ and in ice with thicknesses ranging from 2 to $10 \mathrm{~mm}$ to observe the detuning effects. The dielectric properties of ice and water, given in table 3.3, were used to simulate this scenario. The return loss as a function of frequency is shown in figure 3.11. It is apparent that the bare antenna becomes detuned completely; the center frequency shifts to $8.58 \mathrm{GHz}$ with $10 \mathrm{~mm}$ of ice and $1.78 \mathrm{GHz}$ with 
$4.4 \mathrm{~mm}$ of water. It is for this reason that a radome is often used in outdoor antenna applications and is a good first defense against dielectric detuning. Although the radome helps greatly to isolate the antenna from precipitation it still shows a modest amount of detuning, especially with water due to its high dielectric constant.

Table 3.3: Dielectric Properties of Water and Ice

\begin{tabular}{cccc}
\hline \hline State & Permittivity & Loss Factor & Loss Tangent \\
\hline Water $\left(25^{\circ} \mathrm{C}\right)$ & 78 & 12.9 & 0.16 \\
Ice & 3.2 & 0.0029 & 0.0009 \\
\hline
\end{tabular}

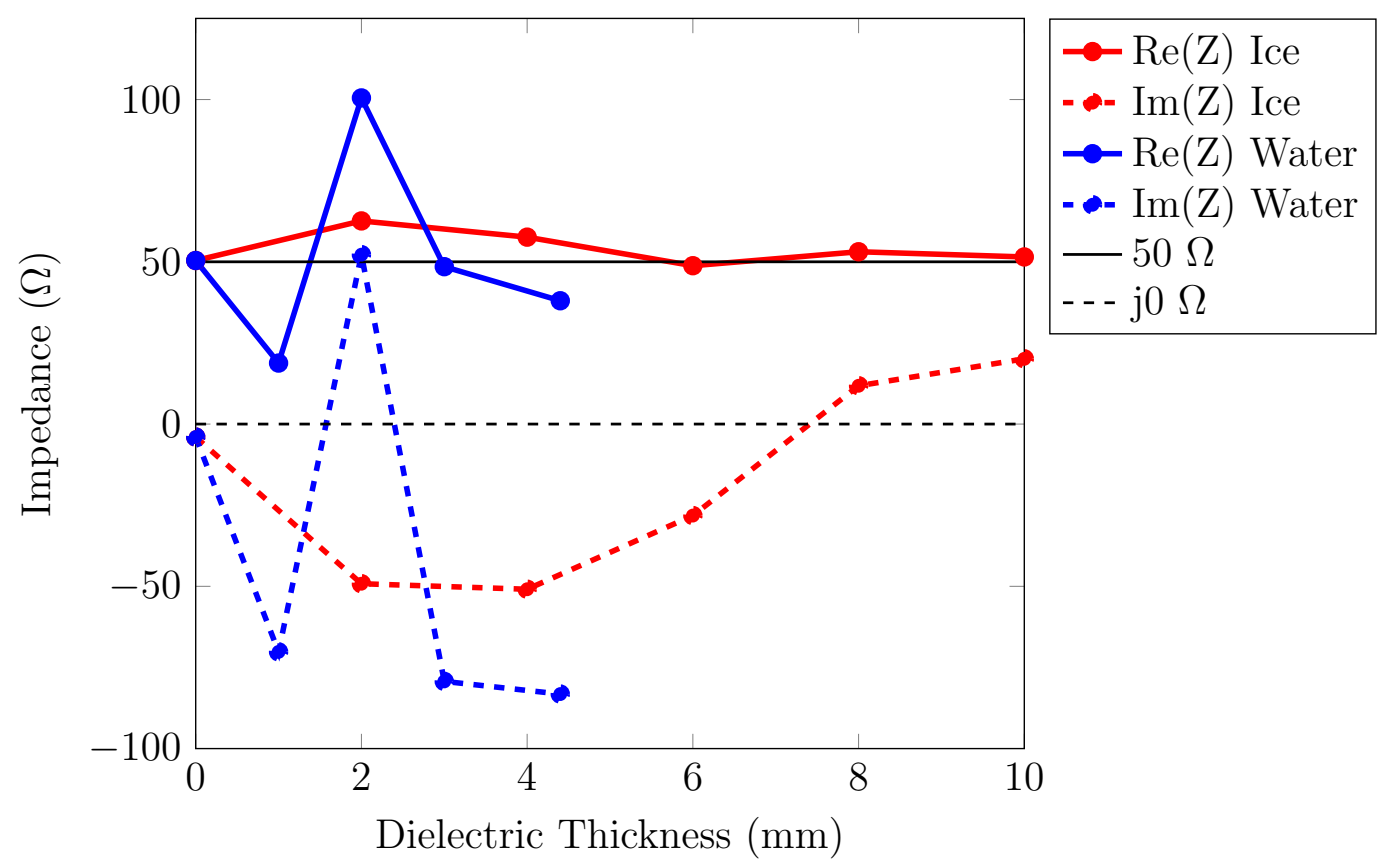

Figure 3.12: Radome covered antenna impedance as a function of ice and water layer thickness.

The impedance of the radome covered antenna input is displayed in figure 3.12. The feed port at the input is a $50 \Omega$ source and the cases with no water or ice layers $(0 \mathrm{~mm}$ thickness $)$ show close to the optimal matching of $(50+\mathrm{j} 0) \Omega$. The total detuning results in a resistive range of 18.8 to $100.5 \Omega$ and reactive range of -83.2 to 


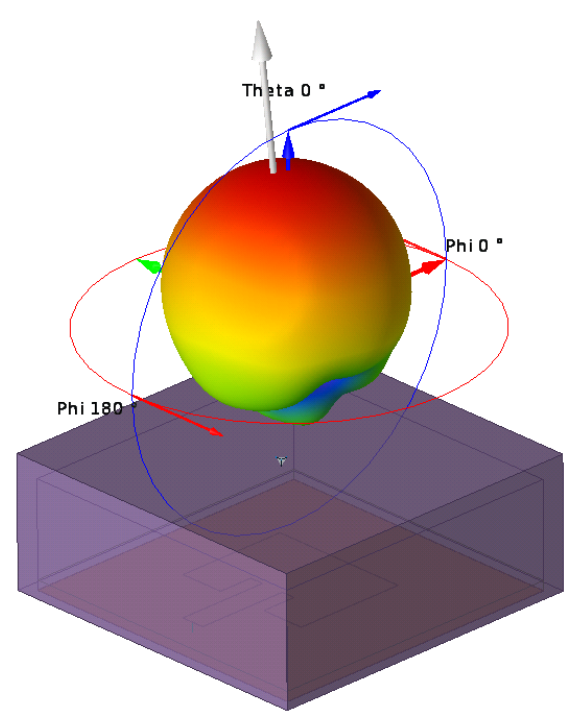

(a) Without water.

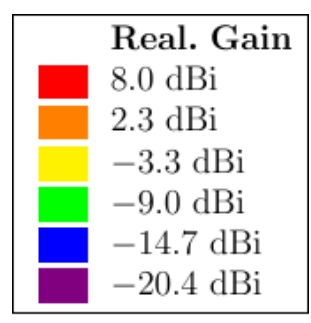

Figure 3.13: Far-field radiation patterns.

To observe the effect of the impedance mismatch on antenna performance one needs to look at the radiation pattern and gain of the antenna. Figure 3.13 shows the realized gain far-field radiation pattern of the radome covered antenna with and without a $4.4 \mathrm{~mm}$ water layer. However, this illustration makes it difficult to isolate the losses due to the impedance mismatch from losses due to a lower radiation efficiency. For that the gain and realized gain need to be compared.

The directivity of an antenna is the amount of (normalized) energy emitted from the antenna in any particular direction.

$$
D(\theta, \phi)=\frac{U(\theta, \phi)}{U_{0}}=\frac{4 \pi U(\theta, \phi)}{P_{\text {rad }}}
$$


where $P_{\text {rad }}$ is the radiated power and $U(\theta, \phi)$ is the radiation intensity as a function of spherical coordinates $\theta$ and $\phi$, and $U_{0}$ is the maximum radiation intensity. Antenna gain is defined as

$$
G(\theta, \phi)=\frac{4 \pi U(\theta, \phi)}{P_{\text {in }}}=\frac{P_{\text {rad }}}{P_{\text {in }}} D(\theta, \phi)=e_{\text {rad }} D(\theta, \phi)
$$

where $P_{i n}$ is the power at the antenna input terminals and $e_{\text {rad }}$ is the radiation efficiency. When the angle is not specified directivity implies maximum directivity (in the direction of the main beam), thus $G=e_{\text {rad }} D$. However, gain does not take into account the mismatch losses at the input. Therefore, the actual or realized gain will be reduced by a factor of $1-|\Gamma|^{2}$ where $|\Gamma|$ is the reflection coefficient. The realized gain is thus

$$
G_{\text {realized }}=\left(1-|\Gamma|^{2}\right) e_{\text {rad }} D=\left(1-|\Gamma|^{2}\right) G
$$

The difference between gain and realized gain will show the losses only related to the impedance mismatch as seen in figure 3.14. The maximum observed mismatch loss is $-10.8 \mathrm{~dB}$ with a $3 \mathrm{~mm}$ water layer, which could potentially be recovered by adaptively matching the input of the antenna. 


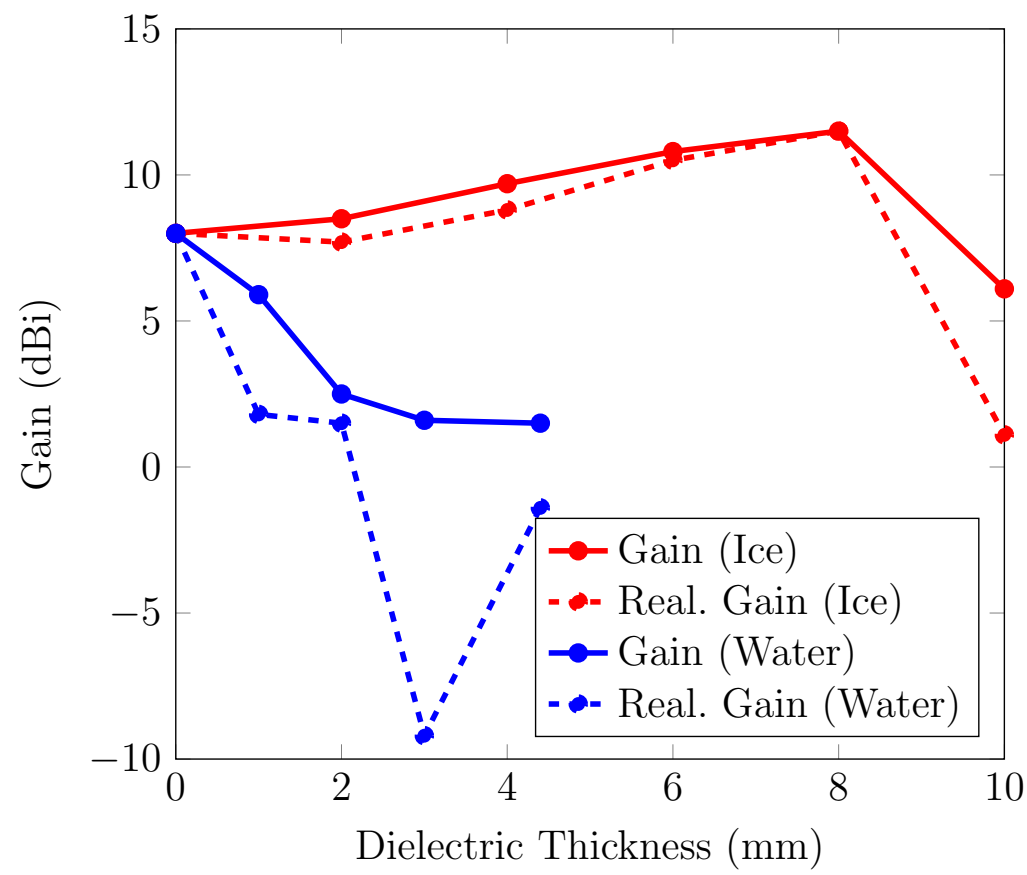

Figure 3.14: Antenna gain and realized gain.

\subsection{Programmable Impedance Matching Network Design}

The PIMN needs to maximize Smith chart coverage to be capable of matching a wide array of antenna impedances. In a switch-capacitor tuner this is accomplished by maximizing the capacitance ratio between $\mathrm{ON}$ and OFF states. The simulated I-V curves have shown that the device stops conducting for a gate bias of $-5 \mathrm{~V}$, which is taken as the OFF state condition, and is well conducting and saturated with a gate bias of $0 \mathrm{~V}$, which is taken as the $\mathrm{ON}$ state condition. The switch capacitor structure ON and OFF state capacitances and Q-factors are simulated for each of the device sizes available and the results are displayed in table 3.4. The HEMT drain is connected to a fixed capacitor sized to $420 \mathrm{fF}$ to provide a capacitance ratio of nearly 3:1. Larger capacitors may be used but would further degrade the Q-factor 
according to (2.32). The maximum capacitance ratio is obtained for a $100 \mu \mathrm{m}$ wide device, with either 2 or 4 fingers. The fabrication process requires that the HEMTs are placed horizontally which places a restriction on the unit cell spacing; therefore, the $4 \times 25 \mu \mathrm{m}$ device was used due to the specific layout concerns encountered here. The switch-capacitor unit cell is mirrored as in figure 2.19 to provide double the tuning range per cell and for design symmetry.

Table 3.4: Switch-Capacitor Capacitance Ratio and Q factor

\begin{tabular}{ccccccc}
\hline \hline Fingers & Finger Width & $C_{O N}(f F)$ & $C_{O F F}(f F)$ & $C_{O N} / C_{O F F}$ & $Q_{O N}$ & $Q_{O F F}$ \\
\hline 2 & 25 & 107 & 43 & 2.47 & 2.2 & 3.6 \\
2 & 50 & 231 & 79 & 2.94 & 2.0 & 3.9 \\
4 & 25 & 231 & 79 & 2.94 & 2.0 & 3.9 \\
2 & 100 & 340 & 134 & 2.54 & 2.8 & 4.5 \\
4 & 50 & 339 & 134 & 2.54 & 2.8 & 4.6 \\
8 & 25 & 339 & 134 & 2.54 & 2.8 & 4.6 \\
2 & 200 & 389 & 204 & 1.91 & 4.9 & 5.7 \\
4 & 100 & 388 & 204 & 1.90 & 4.9 & 6.0 \\
8 & 50 & 388 & 204 & 1.90 & 4.9 & 6.0 \\
4 & 200 & 405 & 275 & 1.47 & 9.5 & 8.5 \\
8 & 100 & 404 & 275 & 1.47 & 9.4 & 8.9 \\
8 & 200 & 410 & 332 & 1.23 & 19.0 & 14.2 \\
\hline
\end{tabular}

The shunt capacitance of the switch-capacitor cell moves the impedance along a single circular path in the bottom half of the Smith chart. A series inductance is added to the input and output of the cell to allow a transition to the top half of the Smith chart and between shunt capacitance impedance circles for greater impedance coverage. The length of transmission line between unit cells is chosen to provide an impedance roughly equal to that of the shunt capacitance at its maximum value in the ON state, which is equal to the fixed capacitor value, or twice that value for a 


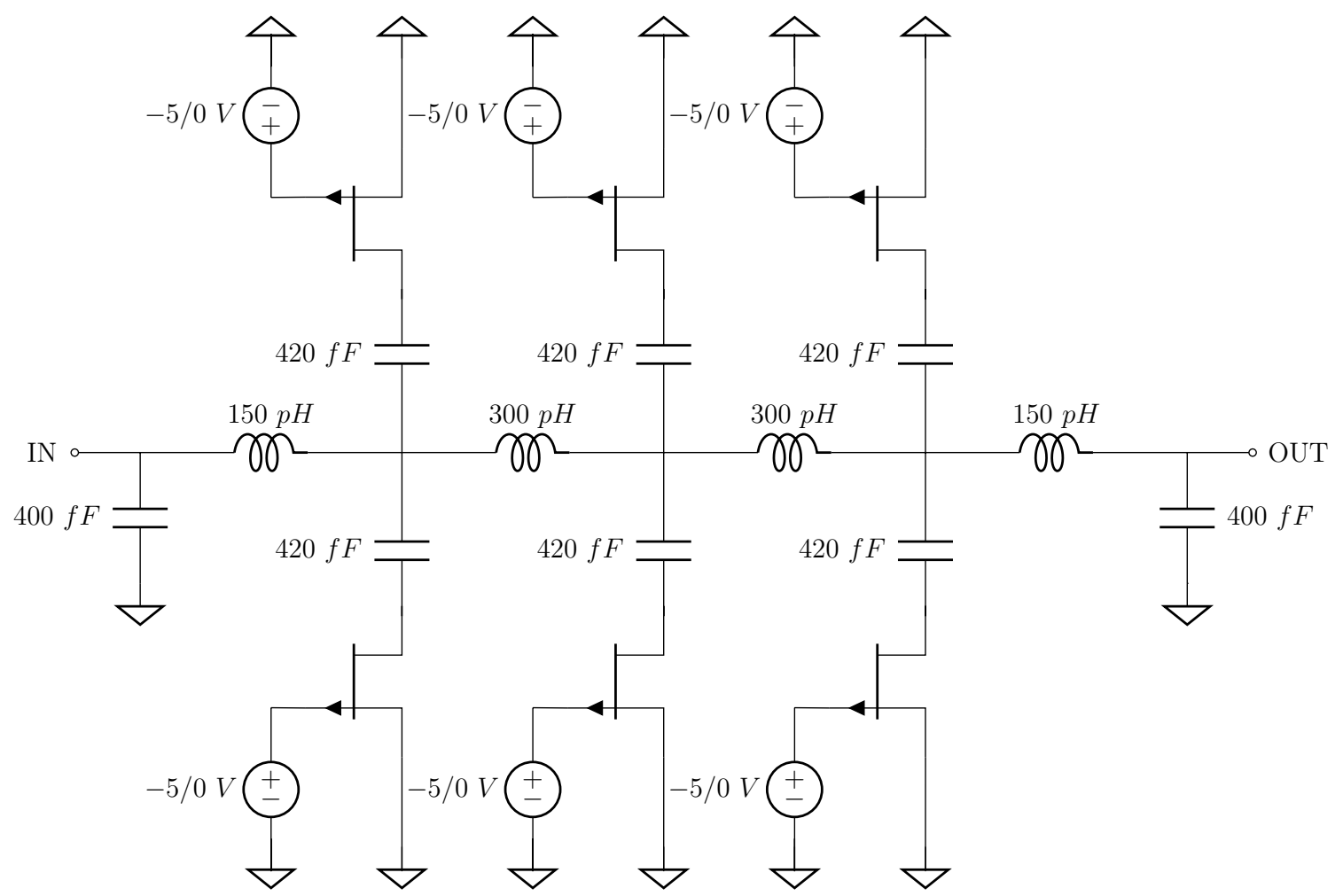

Figure 3.15: 3-bit programmable tuner schematic.

mirrored configuration.

$$
\begin{aligned}
\left|Z_{L}\right| & \sim\left|Z_{C}\right| \\
\left|j \omega L_{\text {total }}\right| & \sim\left|\frac{1}{j \omega C_{\text {total }}}\right| \\
2 L_{t} & =\frac{1}{(2 \pi f)^{2} \cdot 2 C_{L}} \\
L_{t} & =\frac{1}{\left(2 \pi 10^{10} \mathrm{GHz}\right)^{2} \cdot 420 \mathrm{fF}} \\
& =150 \mathrm{pH}
\end{aligned}
$$

The mirrored switch-capacitor cell is cascaded to give the full PIMN circuit seen in figure 3.15. Given the length of transmission lines required to realize this inductance 
three mirrored switch-capacitor section are able to fit within the allotted chip space, with three bits (T1, T2, T3) allowing for $2^{3}=8$ impedance states. S-parameters of the 3-bit network are $S_{11}=S_{22}=0.18 \angle 131^{\circ}$ and $S_{12}=S_{21}=0.83 \angle-77^{\circ}$. For a simultaneous conjugate match to $50 \Omega$ a $400 \mathrm{fF}$ shunt capacitor is added to the input and output of the PIMN. The filter topology of the two-stage PA is high-pass while the PIMN has a low-pass topology which will give the circuit an overall bandpass characteristic.

\subsection{Layout}

The PA and PIMN network schematics were connected together and the matching component values were optimized for maximum $P A E$ using a quasi-Newton algorithm. Then the ideal lumped capacitors and resistors were replaced by their design kit equivalents and inductors were replaced by a length of transmission line.

A CPW interconnect structure is needed because the process does not support through hole vias nor does it have a back conducting plane, and CPW has the advantages outlined in section 2.6 that make it preferable to microstrip. EM co-simulation is performed using the Momentum simulator available in ADS which is a 3D planar EM simulator that uses Method of Moments to find S-parameters for all metalization layers. The maximum allowable span of the airbridge is $100 \mu \mathrm{m}$ which limits the range of conductor widths and gaps of the CPW. Figure 3.16 shows the simulated structure, and the characteristic impedance and inductance as a function of the cen- 


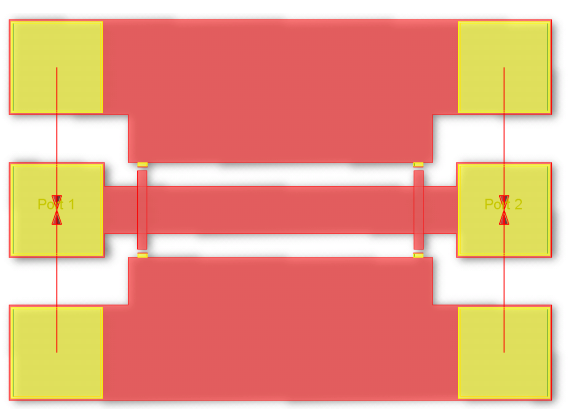

(a) Simulated CPW structure.

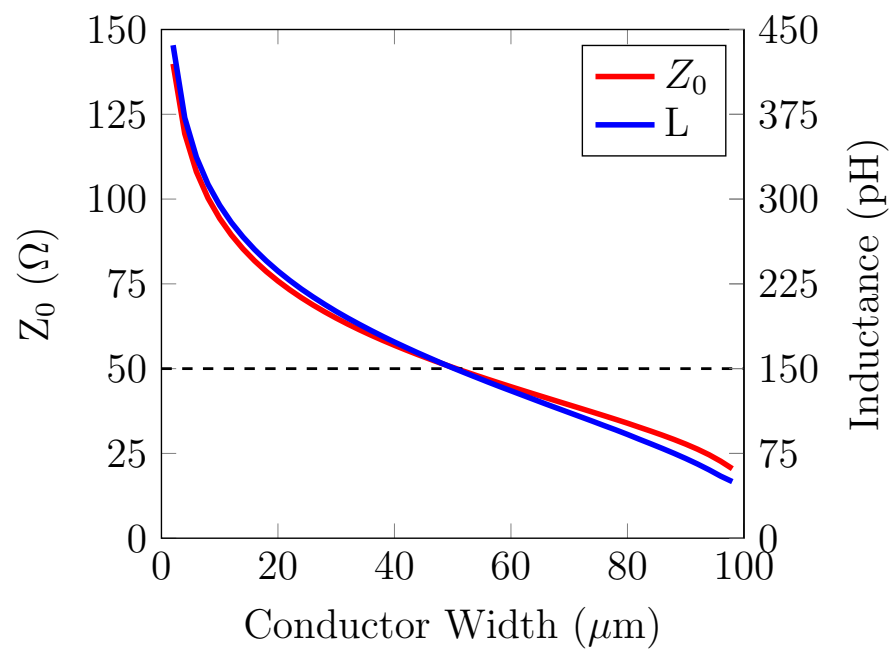

(b) Characteristic Impedance.

Figure 3.16: Simulated CPW characteristic impedance and inductance.

ter conductor width. The ground plane to ground plane spacing is maintained at the maximum of $100 \mu \mathrm{m}$. A $50 \Omega$ characteristic impedance and $150 \mathrm{pH}$ inductance is achieved with a $50 \mu \mathrm{m}$ conductor width and $25 \mu \mathrm{m}$ gap with a line length of $370 \mu \mathrm{m}$.

The circuit EM simulation is performed by removing all discrete components including HEMTs, capacitors, and resistors with only the transmission lines and ground planes left in place. Ports are placed at each junction and S-parameters are simulated from 1 to $50 \mathrm{GHz}$ (4 harmonics). The simulated metal structure is placed as a component in the ADS schematic and the discrete components are connected and cosimulated with the structure. Extensive optimization and reiteration was performed to maximize $P A E$. Since the inductors are transmission line components they must be simulated using the EM Momentum simulator to obtain the greatest accuracy, but this requires significant computing resources for a high density circuit with many 


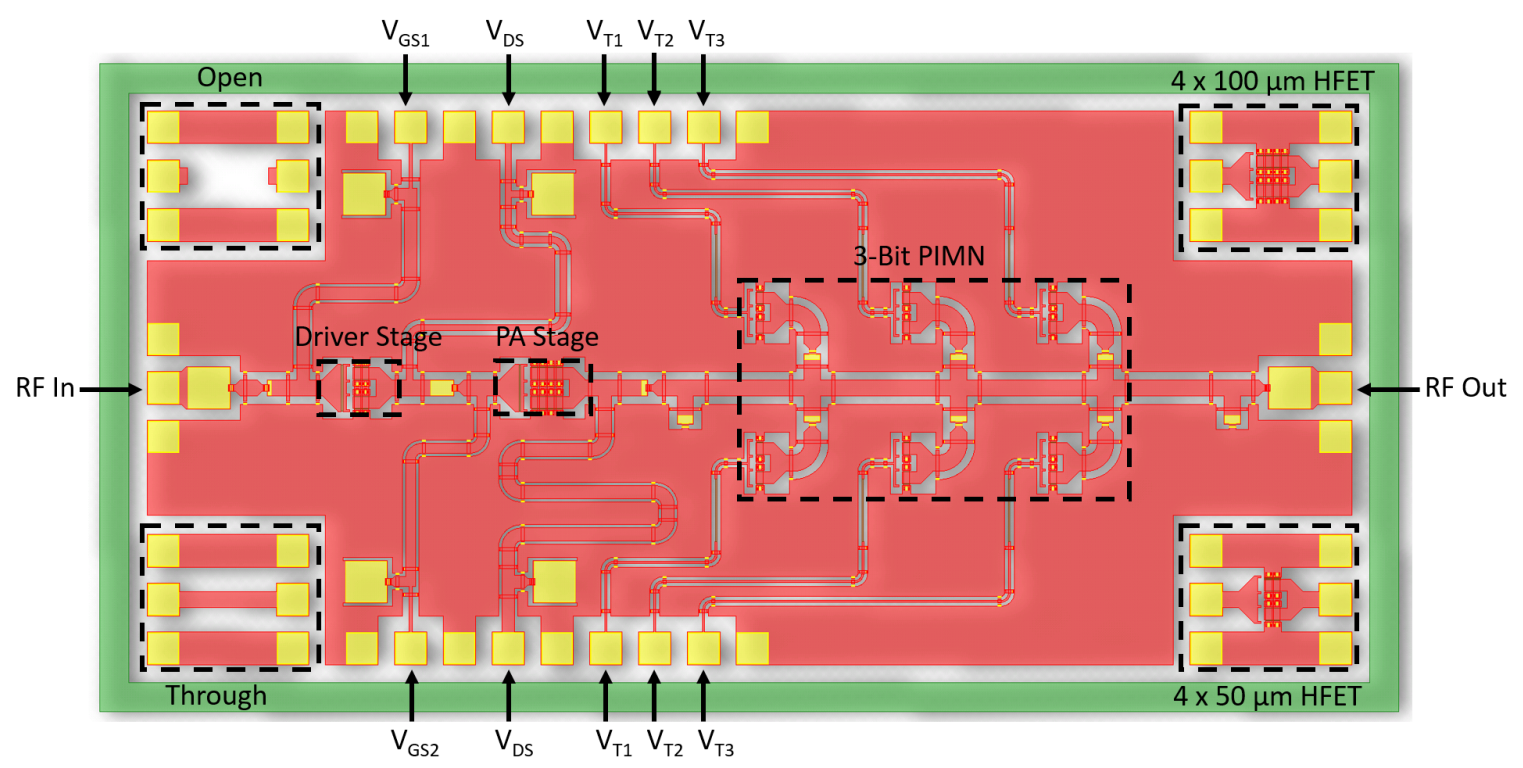

Figure 3.17: GaN chip layout.

ports.

Figure 3.17 shows the finalized PA and PIMN layout. The probe landing pads are $100 \times 100 \mu \mathrm{m}^{2}$ in size with a $150 \mu \mathrm{m}$ pitch. DC blocking capacitors are placed at the input and output of the circuit. Four test measurement cases are placed in the corners of the chip. These include the Open, $50 \Omega$ through, and the two HEMTs used in the PA design for characterization.

The final co-simulation results are shown in figures 3.18 - 3.21. 2-port S-parameters are swept from 6.5 to $13.5 \mathrm{GHz} . S_{11}$ is well matched at $10 \mathrm{GHz}$ with a return loss of $21.6 \mathrm{~dB}$, exceeding the specification of $12 \mathrm{~dB} . S_{22}$ is not as well matched but still meets the specification, with a maximum return loss of $18.3 \mathrm{~dB}$ and is shifted up in frequency to $11 \mathrm{GHz}$, but this is expected as the PA output was matched for maximum $P A E$ and power, and not conjugate matched to $50 \Omega . S_{12}$ shows a good reverse 


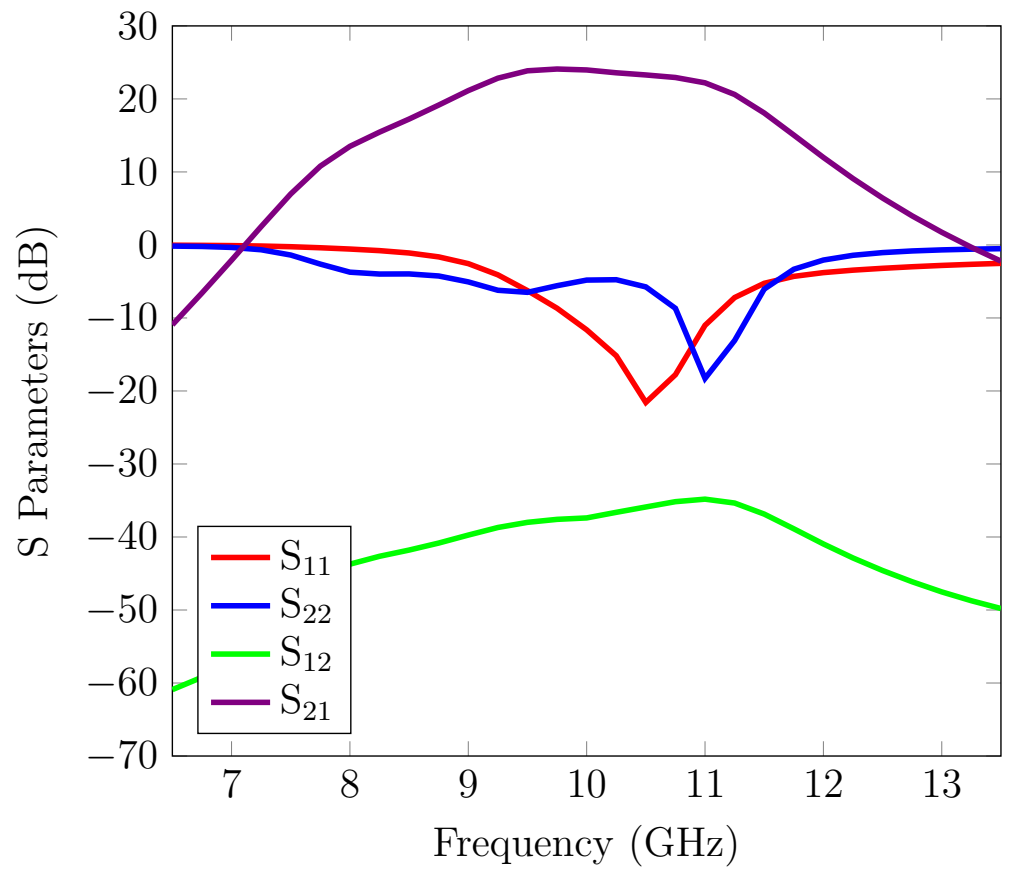

Figure 3.18: PA simulated S parameters.

isolation of $34.8 \mathrm{~dB}$ or better. $S_{21}$ shows a bandpass characteristic and a small-signal gain of $24.0 \mathrm{~dB}$ at $10 \mathrm{GHz}$ with a $3 \mathrm{~dB}$ bandwidth of $2.0 \mathrm{GHz}$.

The output power, large signal gain, and $P A E$ are displayed in figure 3.19 as a function of the input power and were obtained using a $5^{\text {th }}$ order harmonic balance simulation. The amplifier remains linear until an input power of about $10 \mathrm{dBm}$. The $1 \mathrm{~dB}$ compression point occurs at an input power of $11.5 \mathrm{dBm}$ where the output power is $34.5 \mathrm{dBm}$ with a gain of $23.0 \mathrm{~dB}$ and $P A E$ of $33.2 \%$, which exceeds the minimum specifications of $30 \mathrm{dBm}, 12 \mathrm{~dB}$, and $30 \%$, respectively. The saturated output power is $35.6 \mathrm{dBm}$. The maximum $P A E$ point is located after the $1 \mathrm{~dB}$ compression point and is equal to $36.2 \%$ for an input power of $15.5 \mathrm{dBm}$. A two-tone harmonic balance simulation was carried out to find TOI distortion. The frequencies 


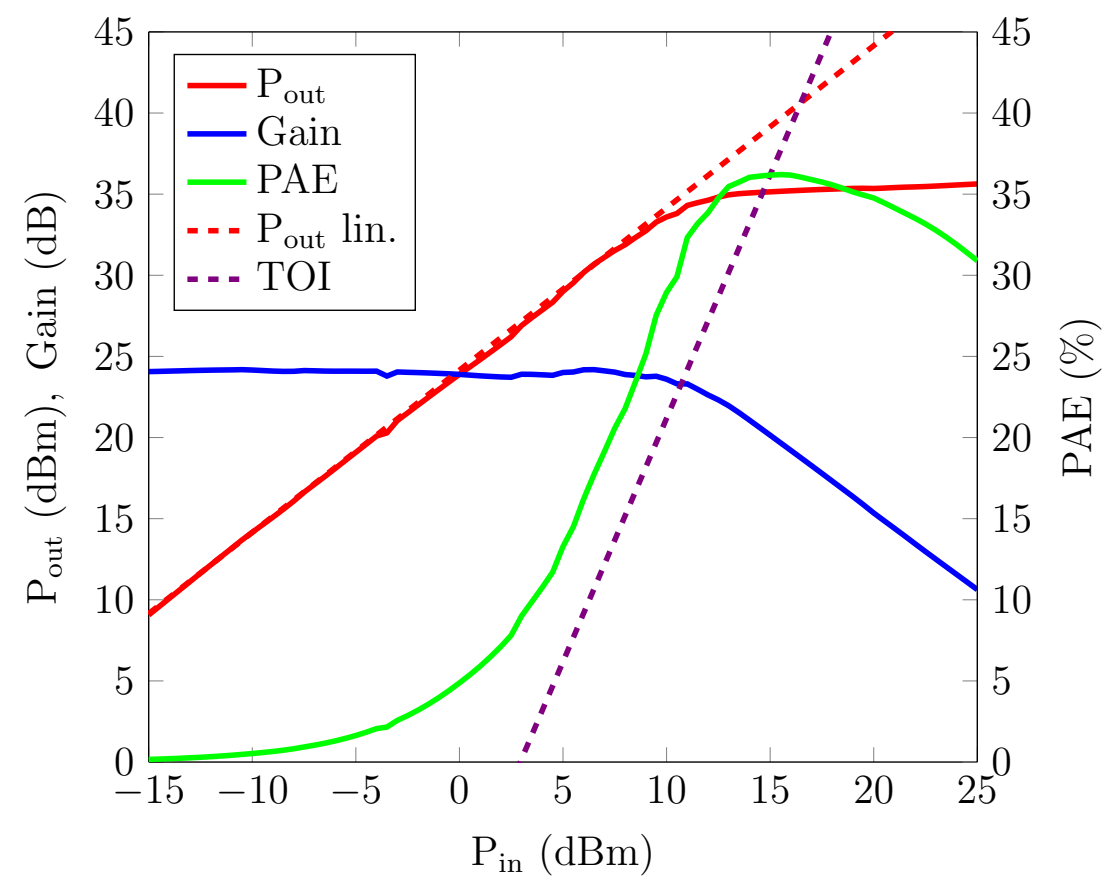

Figure 3.19: Output power, gain and PAE vs input power.

used were $f_{1}=9.95 \mathrm{GHz}$ and $f_{2}=10.05 \mathrm{GHz}$ giving TOI products at $9.9 \mathrm{GHz}$ and 10.1 GHz. The third order intercept (IP3) was found by extrapolating the linear regions of the total power of these tones and the one-tone output power as a function of input power. The OIP3 and IIP3 were found to be $40.7 \mathrm{dBm}$ and $16.5 \mathrm{dBm}$, respectively.

Figure 3.20 shows the current and voltage output waveforms of the PA. Maximum excursions for the voltage are $-19.1 \mathrm{~V}$ and $18.4 \mathrm{~V}$, and for the current are $-382 \mathrm{~mA}$ and $369 \mathrm{~mA}$. The waveform shows some distortion due to non-linearities arising from the reduced conduction angle of the single-ended class AB output stage.

Figure 3.21 shows the normalized impedance range of the PA with the output PIMN for each of the eight possible states. The output impedance has a resistive 


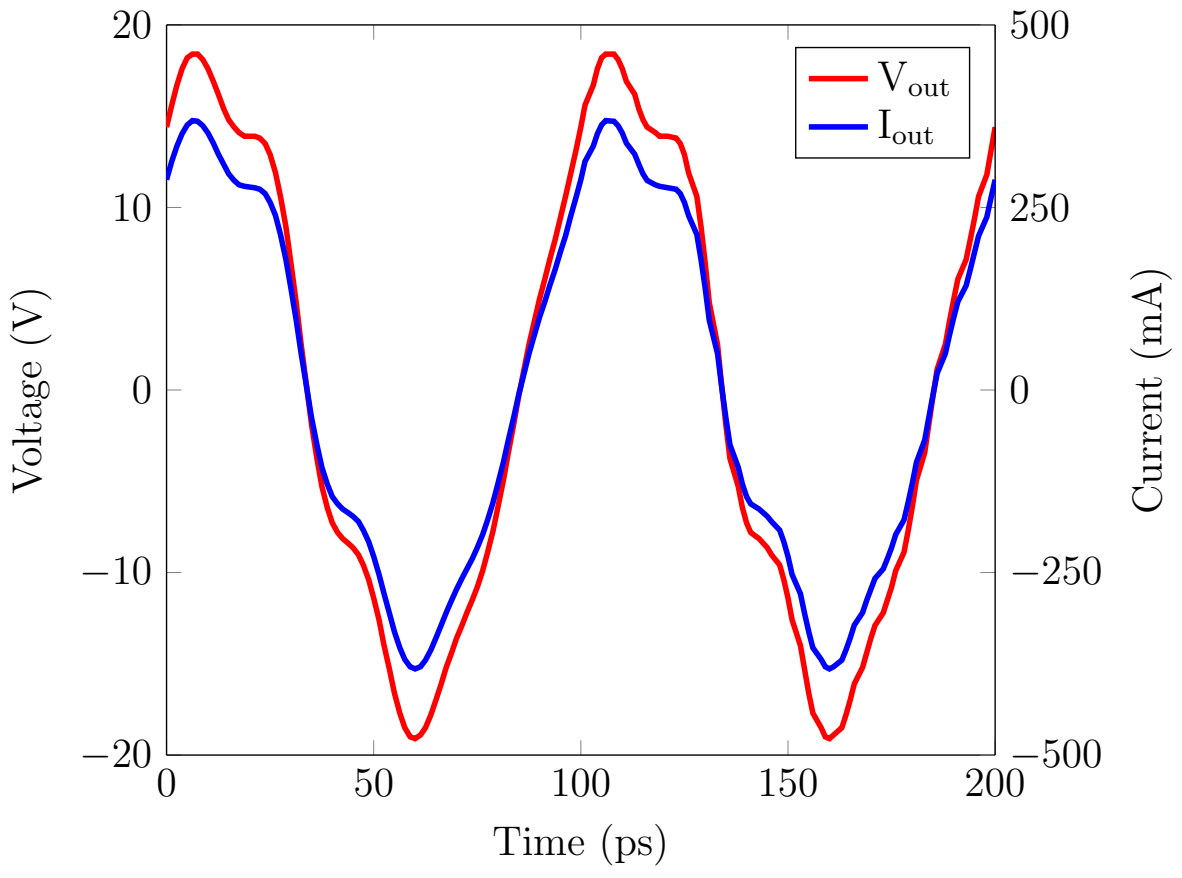

Figure 3.20: PA simulated current and voltage waveforms.

range from 12.4 to $32.3 \Omega$ and a reactive range from -19.1 to $3.7 \Omega$ for a VSWR ratio of 2.1. Since $S_{12} \neq 0$ the input impedance is also slightly shifted for each impedance state. Figure 3.22 shows the improvement in realized gain for the radome covered antenna and the optimal three-bit tuner impedance state (T1, T2, T3) used in each case. The average improvement in linear gain is $34 \%$ and as much as $9.4 \mathrm{~dB}$ for a water layer thickness of $3 \mathrm{~mm}$. 


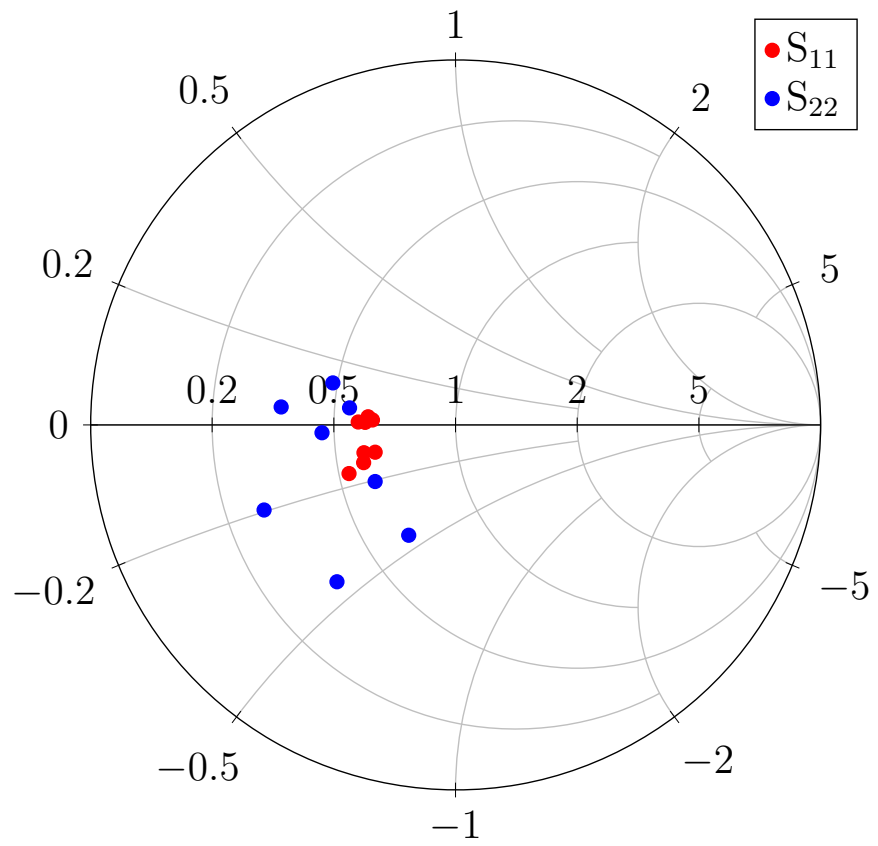

Figure 3.21: Normalized tuner input and output impedance states.

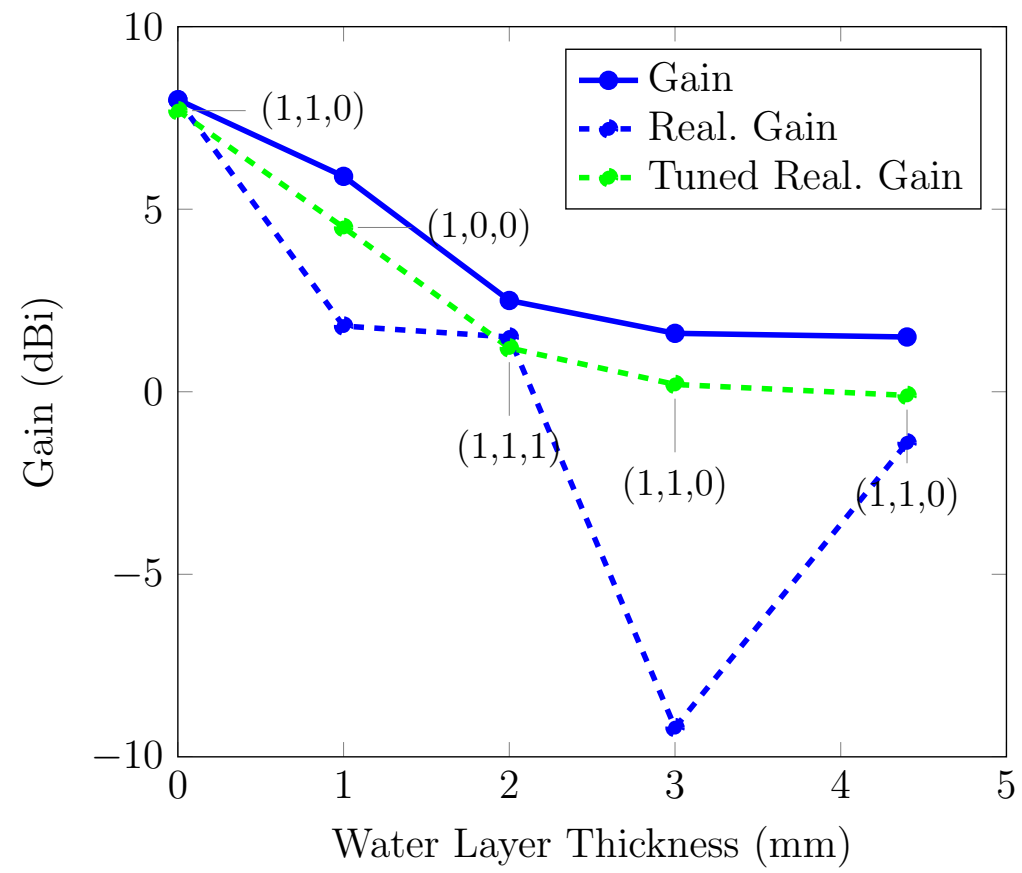

Figure 3.22: Water covered antenna realized gain before and after tuning. 


\section{Implementation}

\subsection{Test Setup and Results}

CMC Microsystems fabricated 26 copies of the chip shown in figure 3.18 which were mounted on a glass surface with epoxy adhesive for testing. The input and output ports were probed with $40 \mathrm{GHz}$ RF probes in a G-S-G (ground-signal-ground) configuration with a $150 \mu \mathrm{m}$ pitch. The DC biasing, which includes the gate and drain bias for each PA stage and the gate bias for each of the three tuner switches, is supplied by six power supplies connected to two DC probes having nine power pins with a $150 \mu \mathrm{m}$ pitch.

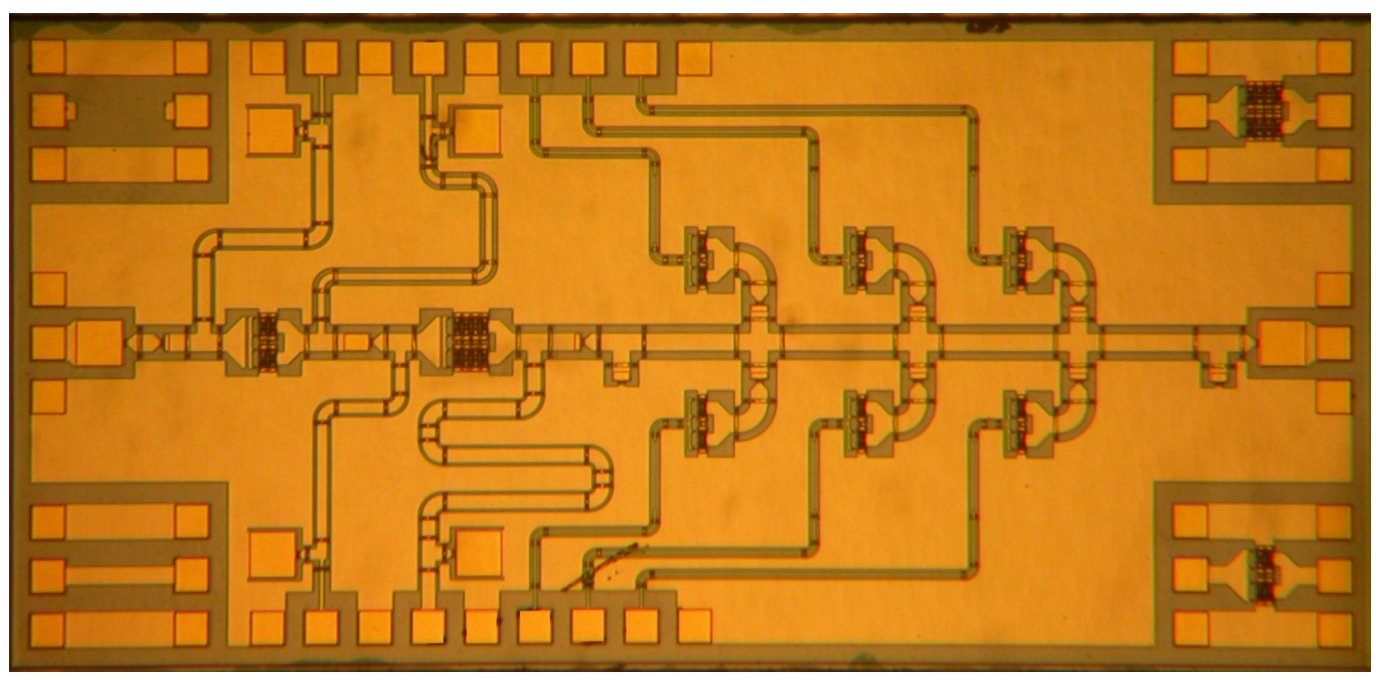

Figure 4.1: Photograph of GaN chip under microscope.

First the I-V curves (figures 4.2 and 4.3) of the bare test devices were measured by sweeping the drain voltage from 0 to $30 \mathrm{~V}$ for a number of gate biases and measuring the current consumption with a multimeter. The probe pins were biased through 
bias tees on the input and output ports of a vector network analyzer (VNA). The current consumption of the $4 \times 100 \mu \mathrm{m}$ FET was found to be roughly twice that of the $4 \times 50 \mu \mathrm{m}$ FET, as expected. Comparing the results to the simulated I-V curves in figure 3.5 shows that the saturation region maximum current is similar $(309 \mathrm{~mA}$ measured compared with $322 \mathrm{~mA}$ in simulation at a gate bias of $0 \mathrm{~V}$ ); however, it displays non-monotonic behaviour with a negative resistance region that causes the current to decline as the drain voltage is increased past the threshold value.

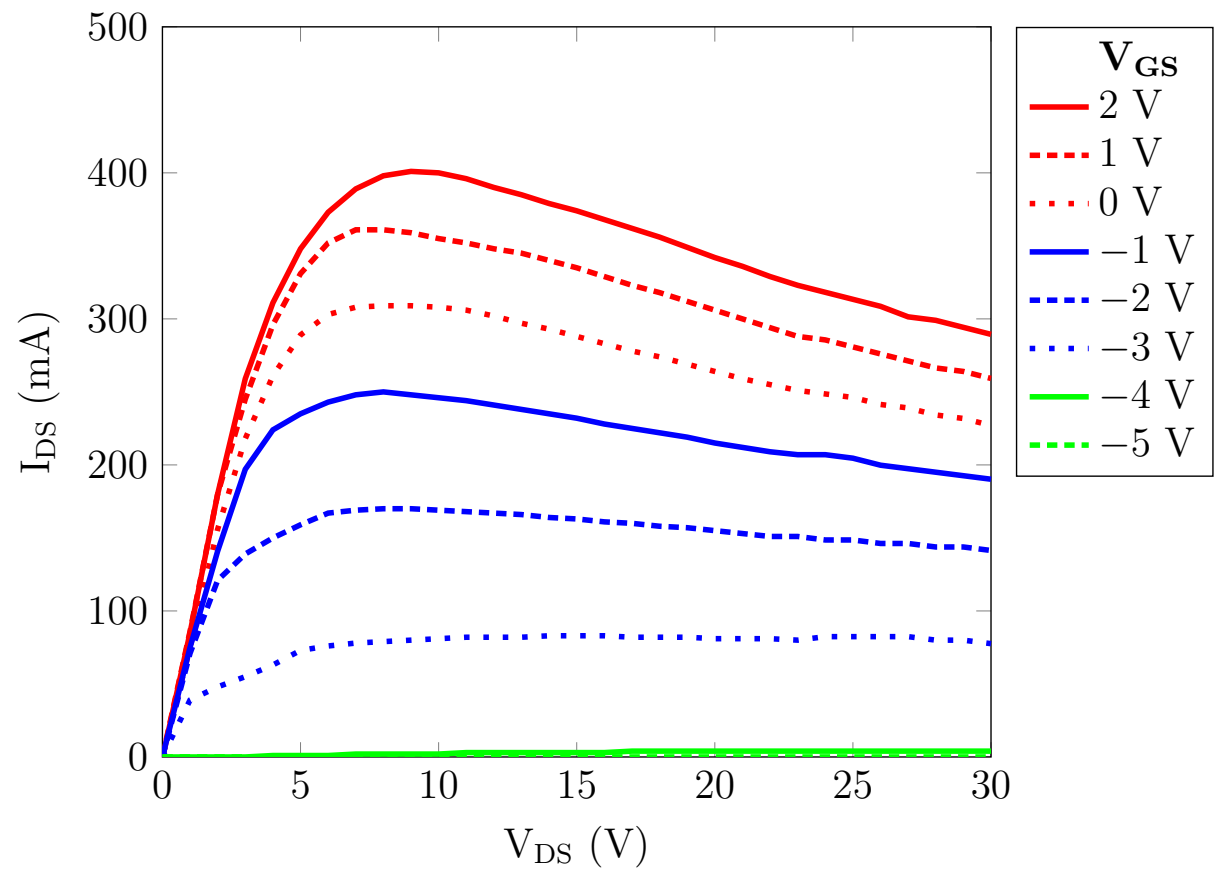

Figure 4.2: Measured $4 \times 100 \mu \mathrm{m}$ FET I-V curves.

Figure 4.4 shows the bare FET measured and simulated S Parameters. Measured results show reasonable agreement with simulations for $S_{12} . S_{11}$ and $S_{21}$ show good agreement at lower frequencies but deviate from simulations at higher frequencies. $S_{22}$ shows a near short circuit condition and does not agree with the simulated values. 


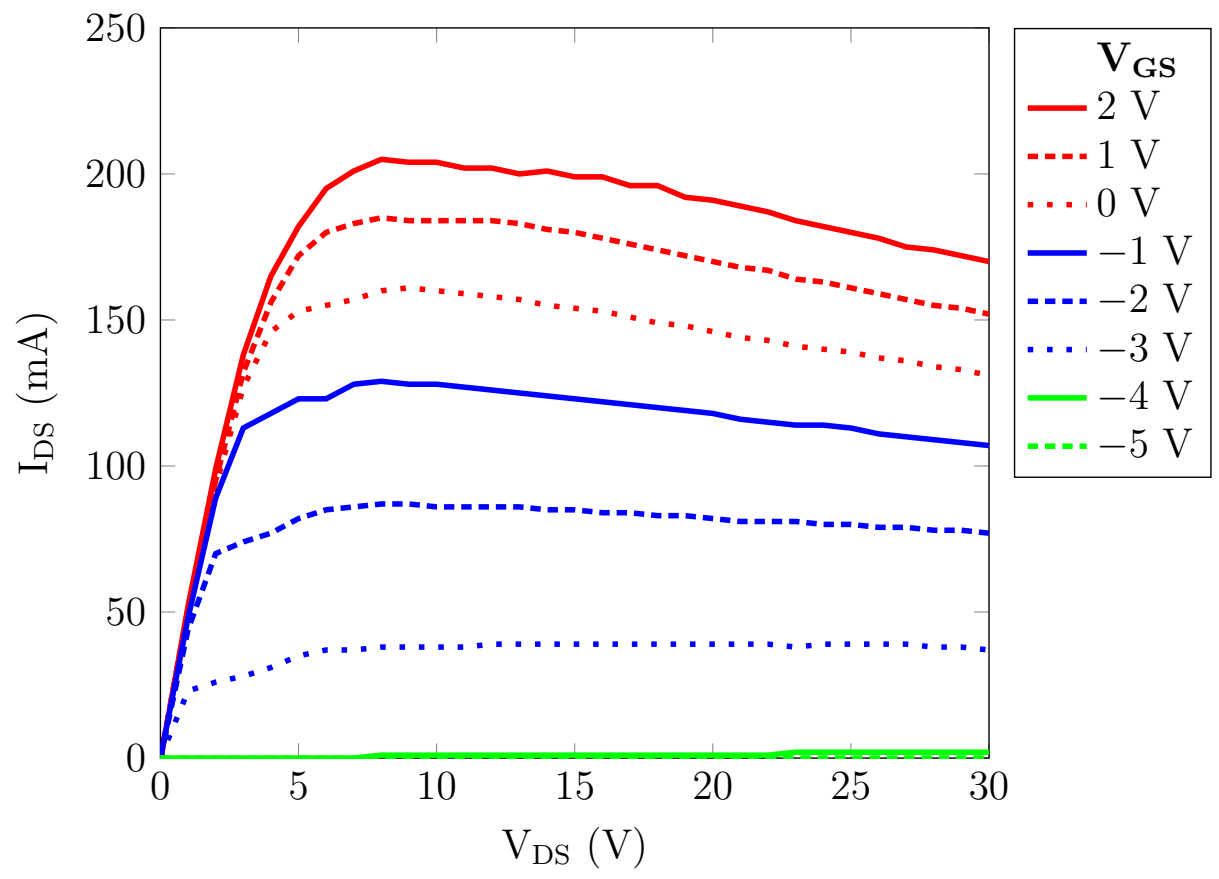

Figure 4.3: Measured $4 \times 50 \mu \mathrm{m}$ FET I-V curves.

S-parameters were measured using a VNA. The input and output of the VNA are calibrated to $50 \Omega$ and $0 \mathrm{~dB}$ attenuation to remove the effects of the cable and $\mathrm{RF}$ probes by using a set of CPW standards. These standards include the 1-port short and open, and 2-port through and $50 \Omega$ load.

The amplifier was found to be unstable for a drain bias of $V_{D S} \geq 2.5 \mathrm{~V}$. Measured S-parameters are shown for this bias level and are compared with simulated values in figure 4.5. The input voltage reflection coefficient $\left(\mathrm{S}_{11}\right)$ shows a return loss of $16.1 \mathrm{~dB}$ at $8.3 \mathrm{GHz}$ and the output voltage reflection coefficient $\left(\mathrm{S}_{22}\right)$ shows a return loss of $29.6 \mathrm{~dB}$ at $8.2 \mathrm{GHz}$. While this is greater than the simulated values of $11.1 \mathrm{~dB}$ and $9.6 \mathrm{~dB}$, respectively, the center frequency is significantly lower than in simulations. The small signal gain $\left(\mathrm{S}_{21}\right)$ is $-9.0 \mathrm{~dB}$ at $8.0 \mathrm{GHz}$ and significantly lower than the 


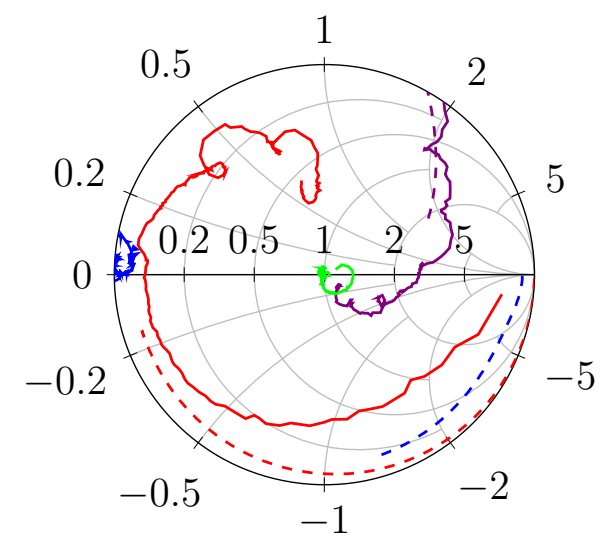

(a) $4 \times 50 \mu \mathrm{m}$.

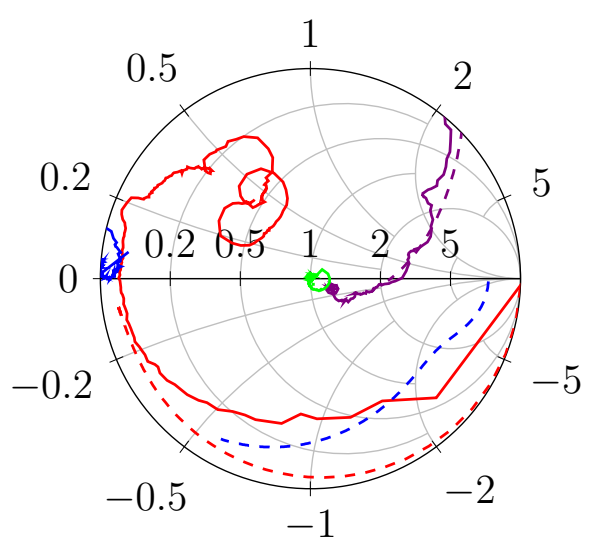

(b) $4 \times 100 \mu \mathrm{m}$.

Figure 4.4: Measured (solid lines) and simulated (dashed lines) FET S parameters.

simulated value of $10.1 \mathrm{~dB}$ at $8.8 \mathrm{GHz}$. The reverse isolation $\left(\mathrm{S}_{12}\right)$ is at least $67.1 \mathrm{~dB}$ across the band while only $22.1 \mathrm{~dB}$ in simulations. The simulations show a region of instability near 7.0 GHz where $\mathrm{S}_{22}$ spikes up above $0 \mathrm{~dB}$. This is not observed in simulations at larger drain biases for which the PA was designed.

To observe what happens as the drain voltage increases beyond $2.5 \mathrm{~V}$ the spectrum of the output was measured using a spectrum analyzer with a $0 \mathrm{dBm}$ input power fed by a signal generator. The spectra in figure 4.6 shows that for a drain bias of 1.3 $\mathrm{V}$ there is a single tone output with gain equal to the measured small signal gain. However, as the voltage is increased to $2.5 \mathrm{~V}$ spurious oscillations occur and continue to rise with the drain voltage. The oscillations continue if the input is disconnected. The PA instability could not be significantly improved by changing the gate biasing or by added external resistors. 


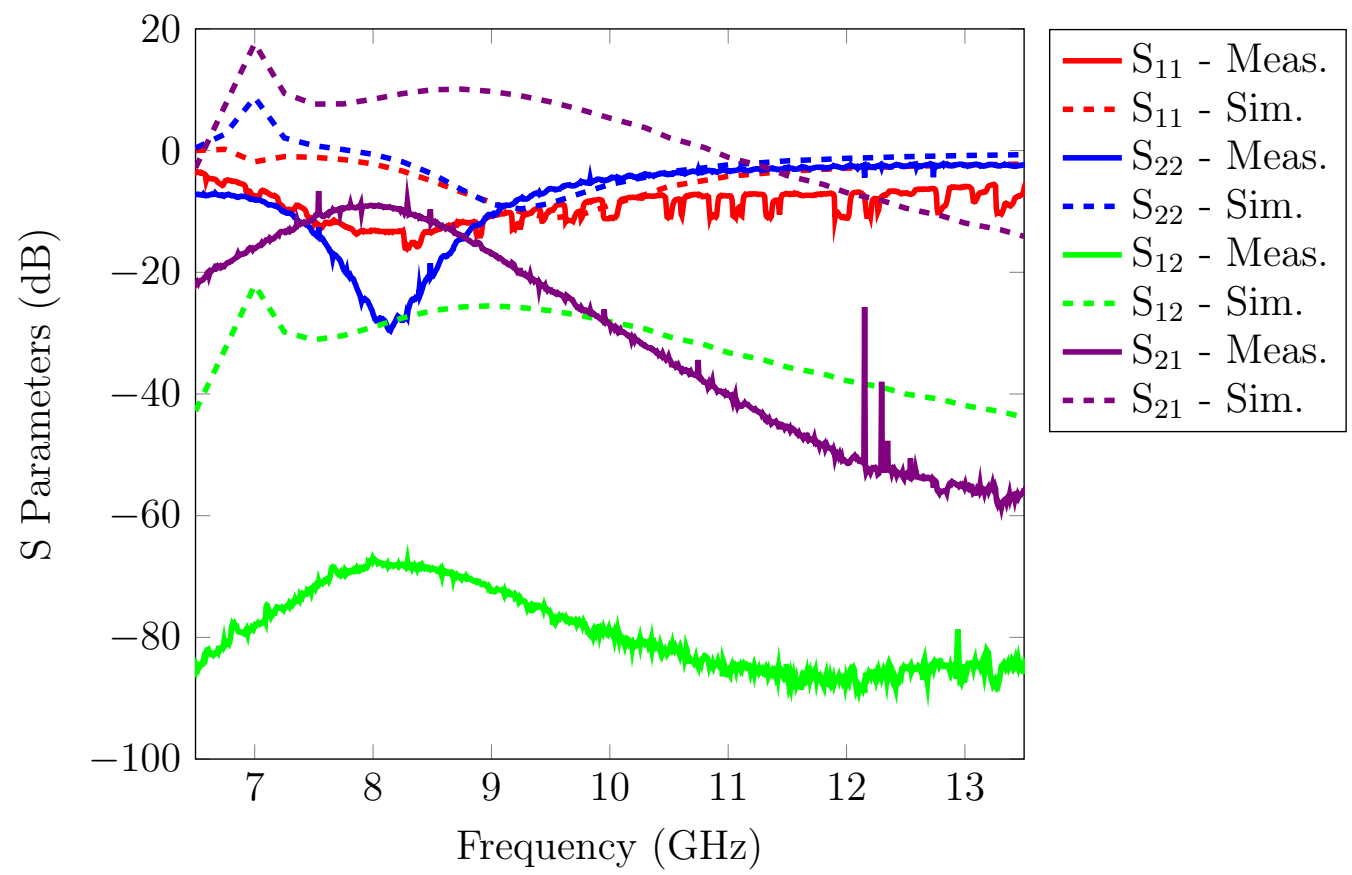

Figure 4.5: PA measured and simulated S parameters with drain bias of $2.5 \mathrm{~V}$.

\subsection{Sources of Instability}

The PA was designed near the edge of stability in order to maximize PAE. The Rollett stability factor was about 1.1 for the driver stage, giving it a small margin of error. Both stages use a series resistor for stability to minimize area giving the Rollett stability factor the high frequency stability characteristic seen in figure 3.4. A shunt resistor shows better low frequency stability as seen in figure 4.7 and should be used instead in future designs although it lowers the maximum available gain.

The accuracy of the simulation results depends on the accuracy of the S-parameters obtained for the circuit CPW structure, which depend on the mesh density of the Momentum simulator and the geometry of the structure. The mesh frequency was set to $50 \mathrm{GHz}$ and the mesh density to 100 cells per wavelength for a cell size of $60 \mu \mathrm{m}$. 


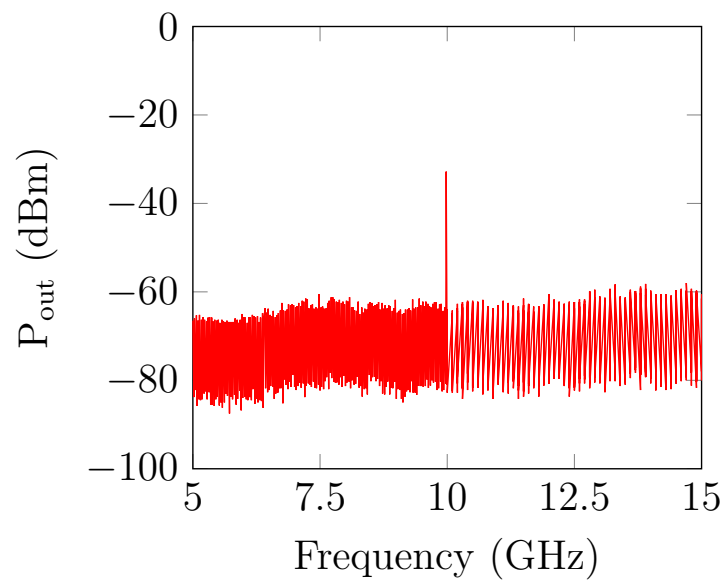

(a) $\mathrm{V}_{\mathrm{DS}}=1.3 \mathrm{~V}$.

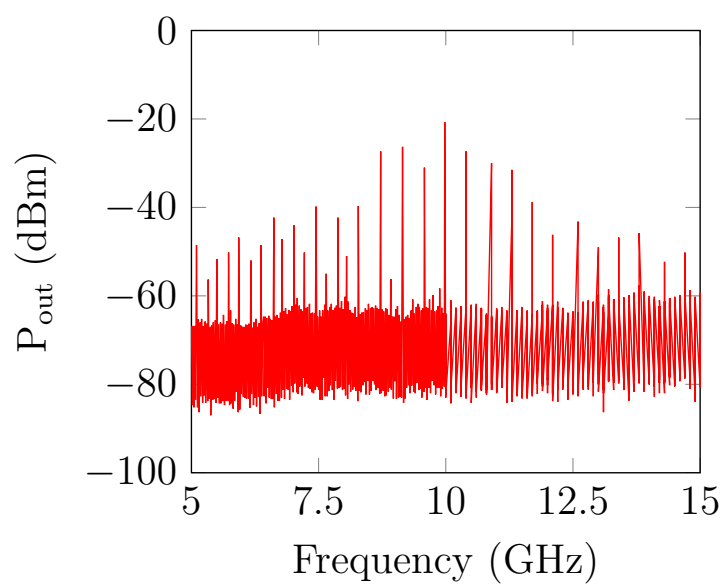

(b) $\mathrm{V}_{\mathrm{DS}}=2.5 \mathrm{~V}$.

Figure 4.6: Output spectrum of PA with input power of $0 \mathrm{dBm}$.

Using $16 \mathrm{CPU}$ cores and $128 \mathrm{~GB}$ of RAM the simulation time was over a day and time constraints prevented the use of higher mesh densities. Since the inductors were formed using CPW the simulation had to be iterated many times to optimize the inductor length, whereas the capacitor sizing could be optimized much faster in the schematic co-simulation. Some of the structure features were 5 to $10 \mu \mathrm{m}$ in size. In addition the large number of ports contributed to the excessive simulation time. A total of 90 ports and 256 pins were used. The Momentum simulator meshes around each pin and the distance between the center conductor and ground pin must be kept under $\lambda / 10$ at the highest simulation frequency. This makes the exact location of the ground pins affect the result, sometimes in a significant way.

The simulation was repeated with a mesh density of 600 cells per wavelength for a cell size of $10 \mu \mathrm{m}$, which required over a week to complete. The stability factor for the driver stage drops below 1 within the band, as seen in figure 3.4 and is the likely 


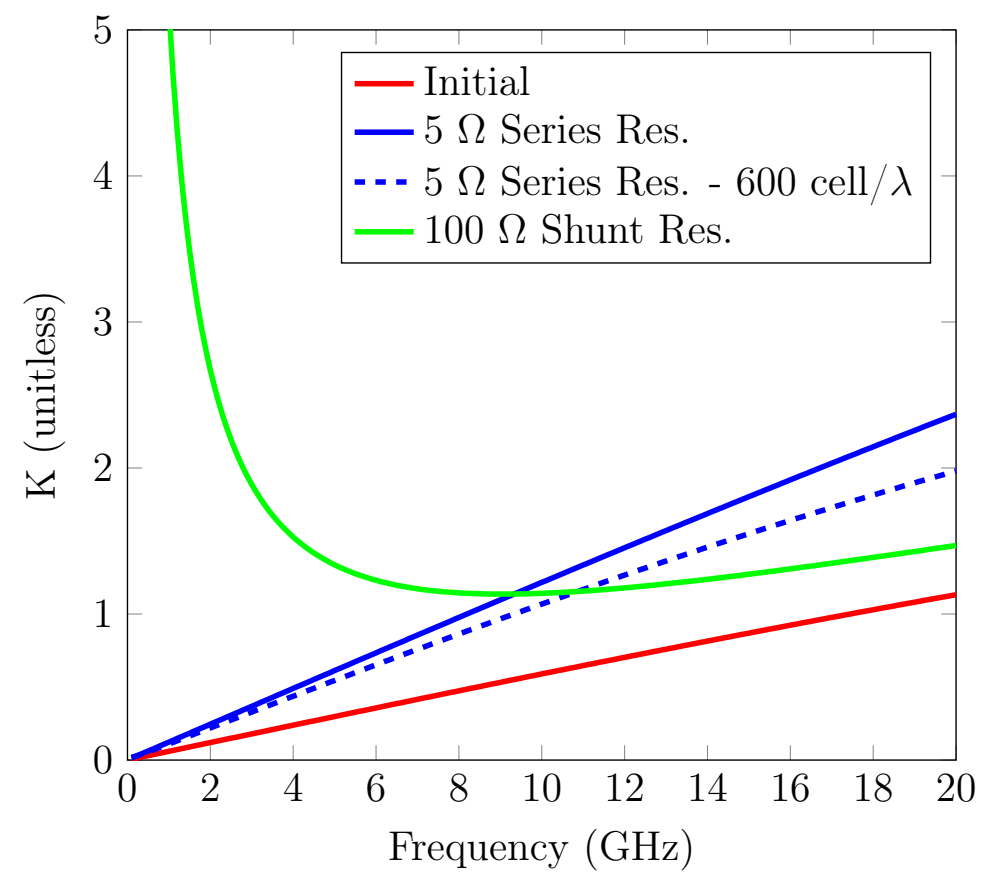

Figure 4.7: Driver stage FET stability factor using series and shunt resistors.

cause of the instability seen in measurements. In figure 4.8 the S-parameters show closer agreement at $10 \mathrm{GHz}$ with the measured results than in the 100 cell per $\lambda$ case. 


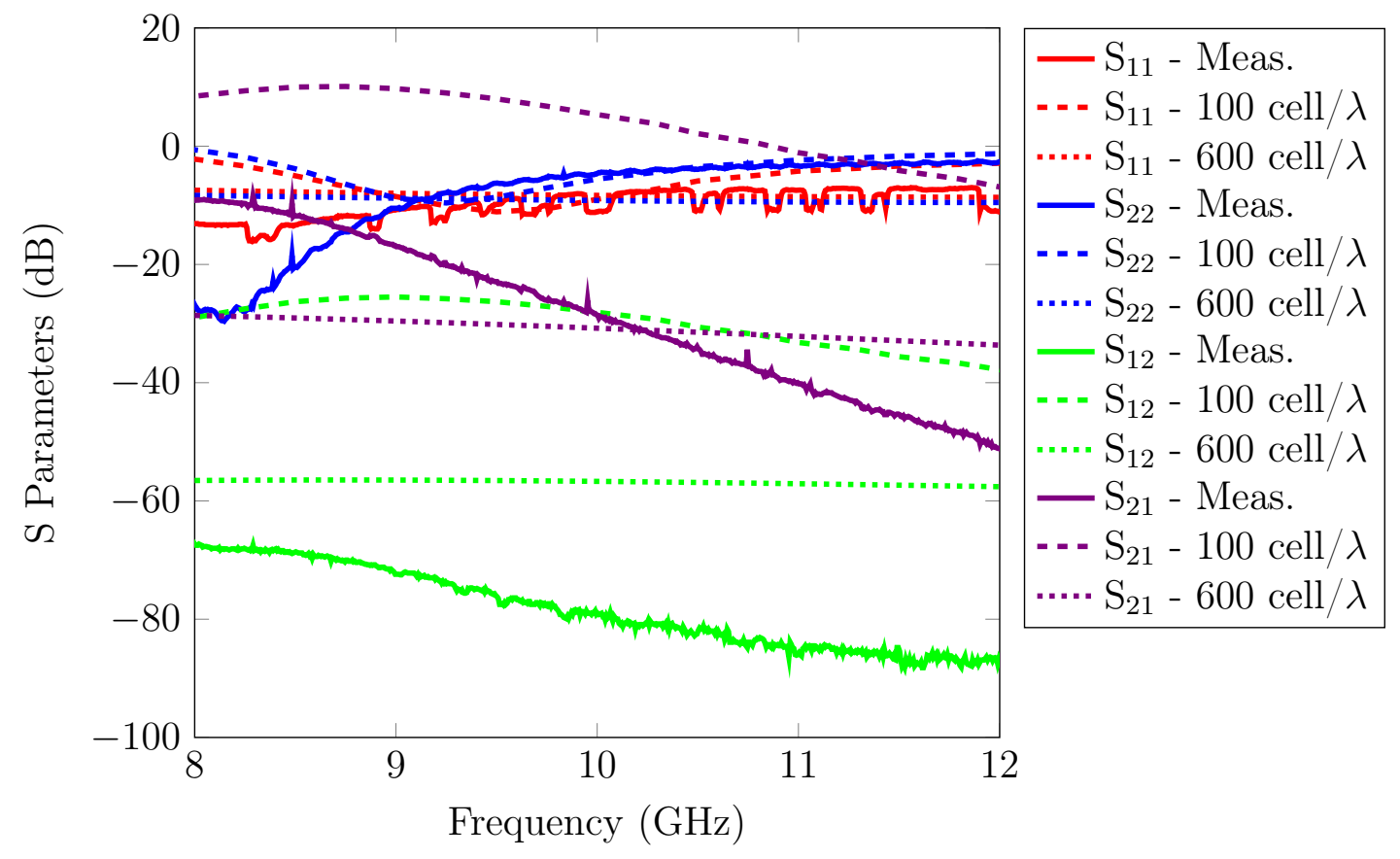

Figure 4.8: PA S parameters with drain bias of $2.5 \mathrm{~V}$ with various mesh densities. 


\section{Conclusions}

\section{$5.1 \quad$ Results}

A GaN PA with an output PIMN was designed and fabricated. A two-stage design was used with a class AB PA stage and a linear class A driver stage to meet the required gain and output power requirements. 3D EM FEM simulations on a microstrip patch antenna designed for X-band were carried out to show the detuning effects of thin layers of ice and water in close proximity to an antenna. Even with a protective radome cover the impedance mismatch can cause up to a $10.8 \mathrm{~dB}$ loss. Thermal expansion can cause an additional detuning effect up to $15 \Omega$ for a $4 \mathrm{~K}$ change in temperature. A three bit PIMN at the output of the PA was designed and matched nominally to $50 \Omega$ to allow adaptive tuning of the antenna input impedance to correct for these situations.

The two circuits were combined together and a 3D planar simulator was used to obtain S-parameters for the CPW interconnect structure and distributed inductors and then the circuit and CPW were co-simulated. The simulations show an output power of $34 \mathrm{dBm}$, with PAE of $33 \%$ and gain of $23 \mathrm{~dB}$ at the $1 \mathrm{~dB}$ compression point, and a saturated output power of $36 \mathrm{dBm}$ and peak PAE of $36 \%$. A twotone test yielded and OIP3 and IIP3 of $40.7 \mathrm{dBm}$ and $16.5 \mathrm{dBm}$, respectively. The output impedance has a resistive range from 12.4 to $32.3 \Omega$ and a reactive range from -19.1 to $3.7 \Omega$ for a VSWR ratio of 2.1 in eight discrete steps, which gave an average 
improvement of $34 \%$ and up to $9.4 \mathrm{~dB}$ in realized gain for a dielectrically detuned radome covered antenna.

The measurements obtained from testing did not match the simulations. The PA was found to have stability issues that could not be resolved without a redesign. After simulating with greater accuracy it was found that the driver stage stability factor would drop below unity within the band and is the likely cause of the instability found in testing.

\subsection{Future Work}

A number of changes to the current design can be made to stabilize the PA and improve performance. First the process will be changed to the NRC GaN500 v3 or GaN150 which will give the improvements outlined in section 2.3. With the increased $f_{t}$ of the GaN150 process, the design can be done at a higher frequency that will allow greater miniaturization. This will also enable a microstrip design which will greatly reduce simulation time by removing the coplanar ground metalization, airbridges, and ground pins but will sacrifice the advantages obtained with a CPW structure outlined in section 2.6.2. The stability network will be changed to a shunt input resistor to give a Rollet stability factor greater than 1.5 from DC up to $f_{\max }$ to ensure stability not only within the band but at all frequencies with potential positive gain and with a good margin of error. The distributed matching inductors will be replaced with discrete spiral inductors to speed up the optimization of the co-simulated circuit. 
More switch-capacitor cells are required to increase Smith chart coverage and step resolution. This would result in the realized gain of the antenna approaching the gain as the number of bits is increased. Using a single sided meandered topology it may be possible to increase this number to six bits for $2^{6}=64$ impedance states within the same $4 \mathrm{~mm} \times 2 \mathrm{~mm}$ area. 


\section{References}

[1] A. A. Huurdeman, The Worldwide History of Telecommunications, ser. A Wileyinterscience publication. Wiley, 2003, pp. 89, 179, 200-201.

[2] R. V. Bruce, Bell: Alexander Graham Bell and the Conquest of Solitude. Cornell University Press, 1990, p. 336.

[3] M. Kennedy, The Global Positioning System and GIS. New York, NY: Taylor \& Francis inc., 2002, pp. 7-8.

[4] L. A. et al., GaN Transistors for Efficient Power Conversion, 2nd ed. Chichester, UK: John Wiley \& Sons, Inc., 2015, p. 3.

[5] C. F. Campbell, A. Balistreri, M. Kao, D. C. Dumka, and J. Hitt, "GaN takes the lead," Microwave Magazine, IEEE, vol. 13, no. 6, p. 44, September 2012.

[6] J. A. Bruder et al., "IEEE standard letter designations for radar-frequency bands," IEEE Std 521-2002 (Revision of IEEE Std 521-1984), p. 2, 2003.

[7] G. A. Siles, J. M. Riera, and P. Garcia-del Pino, "Atmospheric attenuation in wireless communication systems at millimeter and $\mathrm{THz}$ frequencies [wireless corner]," Antennas and Propagation Magazine, IEEE, vol. 57, no. 1, pp. 48-61, February 2015.

[8] I. J. Bahl, Fundamentals of RF and Microwave Transistor Amplifiers. Hoboken, NJ: John Wiley \& Sons, Inc., 2009, pp. 34, 62. 
[9] P. Colantonio, F. Giannini, and E. Limiti, High Efficiency RF and Microwave Solid State Power Amplifiers. Chichester, UK: John Wiley \& Sons, Inc., 2009, pp. 31-32.

[10] T. Erlbacher, Lateral Power Transistors in Integrated Circuits. Cham, CH: Springer, 2014, p. 43.

[11] B. S. Virdee, A. S. Virdee, and B. Y. Banyamin, Broadband Microwave Amplifiers. Norwood, MA: Artech House, 2004, pp. 51, 54-56.

[12] M. Wosko, B. Paszkiewicz, R. Paszkiewicz, and M. Tlaczala, "Influence of AlN spacer on the properties of AlGaN/AlN/GaN heterostructures," Optica Applicata, vol. 43, no. 1, pp. 61-63, 2013.

[13] "Gallium Nitride MMIC Foundry Design Manual - GaN500 Version 1.5," National Research Council Canada, p. 18, 2013.

[14] "Gallium Nitride MMIC Foundry Design Manual - GaN500 Version 2.01," National Research Council Canada, pp. 15,22, 2014.

[15] R. S. Pengelly, S. M. Wood, J. W. Milligan, S. T. Sheppard, and W. L. Pribble, "A review of GaN on $\mathrm{SiC}$ high electron-mobility power transistors and MMICs," Microwave Theory and Techniques, IEEE Transactions on, vol. 60, no. 6, pp. 1764-1783, June 2012.

[16] A. S. Gilmour Jr, Microwave Tubes. Dedham, MA: Artech House, 1986, p. 502. 
[17] D. M. Pozar, Microwave Engineering, 4th ed. Hoboken, NJ: John Wiley \& Sons, Inc., 2012, pp. 49, 147, 234-241, 564 .

[18] J. W. M. Rogers and C. Plett, Radio Frequency Integrated Circuit Design, 2nd ed. Norwood, MA: Artech House, 2010, pp. 19, 214.

[19] T. Sarkar, R. Mailloux, A. Oliner, M. Salazar-Palma, and D. Sengupta, History of Wireless. Wiley-IEEE Press, 2006, pp. 557-558.

[20] M. Houdart, "Coplanar lines: application to broadband microwave integrated circuits," in Microwave Conference, 1976. 6th European, September 1976, p. 50.

[21] I. Wolff, Coplanar Microwave Integrated Circuits. Kamp-Lintfort, Germany: John Wiley \& Sons, Inc., 2006, pp. 2-4, 7-8, 77-78.

[22] P. Singh and A. K. Verma, "Dielectric loss computation of multilayer coplanar waveguide," in Communications and Signal Processing (ICCSP), 2011 International Conference on, February 2011, pp. 428-430.

[23] C. H. Doan, S. Emami, A. M. Niknejad, and R. W. Brodersen, "Millimeter-wave CMOS design," Solid-State Circuits, IEEE Journal of, vol. 40, no. 1, pp. 148-149, January 2005.

[24] L. Rabieirad and S. Mohammadi, "Reconfigurable CMOS tuners for softwaredefined radio," Microwave Theory and Techniques, IEEE Transactions on, vol. 57, no. 11, pp. 2768-2774, Nov 2009. 
[25] C. A. Balanis, Antenna Theory: Analysis and Design, 3rd ed. Hoboken, NJ: John Wiley \& Sons, Inc., 2005, pp. 4-5, 811-825.

[26] K. C. Huang and D. J. Edwards, Millimetre Wave Antennas for Gigabit Wireless Communications. Chichester, UK: John Wiley \& Sons, Inc., 2008, pp. 115-116.

[27] C. A. Balanis, Advanced Engineering Electromagnetics, 2nd ed. Hoboken, NJ: John Wiley \& Sons, Inc., 2012, p. 460.

[28] J. D. Johnson, "Statistical Analysis of Detuning Effects for Implantable Microstrip Antennas," Master of Science, University of Utah, 2007, pp. 9-29.

[29] N. A. Stepanova, "On the lowest temperatures on earth," Monthly Weather Review, pp. 6-8, January 1958.

[30] R. K. Yadav, J. Kishor, and R. L. Yadava, "Effects of temperature variations on microstrip antennas," International Journal of Networks and Communications, pp. 21-24, 2013.

[31] H.-S. Chen, Y.-K. Hsieh, and L.-H. Lu, "A 5.5-GHz multi-mode power amplifier with reconfigurable output matching network," in Radio Frequency Integrated Circuits Symposium (RFIC), 2015 IEEE, May 2015, pp. 203-206.

[32] U. Kim, K. Kim, J. Kim, and Y. Kwon, "A multi-band reconfigurable power amplifier for UMTS handset applications," in Radio Frequency Integrated Circuits Symposium (RFIC), 2010 IEEE, May 2010, pp. 175-178. 
[33] L. Larcher, R. Brama, M. Ganzerli, J. Iannacci, M. Bedani, and A. Gnudi, "A MEMS reconfigurable quad-band class-E power amplifier for GSM standard," in Design, Automation Test in Europe Conference Exhibition, 2009. DATE '09., April 2009, pp. 364-368.

[34] G. Lee, J. Jung, and J.-I. Song, "A multiband power amplifier with a reconfigurable output-matching network for 10-MHz BW LTE Mobile Phone Applications," Circuits and Systems II: Express Briefs, IEEE Transactions on, vol. 62, no. 6, pp. 558-562, June 2015.

[35] H. Maune et al., "Tunable RF GaN-power transistor implementing impedance matching networks based on BST thick films," in Microwave Conference (EuMC), 2012 42nd European, October 2012, pp. 1206-1209.

[36] M. Coffey, S. Schafer, and Z. Popovic, "Two-stage high-efficiency x-band gan mmic pa/ rectifier," in Microwave Symposium (IMS), 2015 IEEE MTT-S International, May 2015, pp. 1-4.

[37] J. Leckey, "A 25w x-band gan pa in smt package," in European Microwave Integrated Circuit Conference (EuMIC), 2014 9th, Oct 2014, pp. 397-309.

[38] "Triquint TGA2624-CP 9 to $10 \mathrm{GHz}, 16 \mathrm{~W}$ GaN Power Amplifier," TriQuint, pp. 1-3.

[39] "Hittite HMC1087F10 8 Watt Flane Mount GaN MMIC Power Amplifier, 2-20 GHz," Hittite Microwave Coporation, p. 3. 
[40] "Hittite HMC7149 10 Watt GaN MMIC Power Amplifier, 6-18 GHz," Hittite Microwave Coporation, pp. 3, 10.

[41] D. M. Dobkin and S. M. Weigand, "Environmental effects on RFID tag antennas," in Microwave Symposium Digest, 2005 IEEE MTT-S International, June 2005, pp. 135-138.

[42] H. Kawakami, G. Sato, and S. Sumihiro, "Characteristics of ice-covered for YagiUda antenna," in Antennas and Propagation Society International Symposium, 1995. AP-S. Digest, vol. 4, June 1995, pp. 1871-1874.

[43] P. G. de Gennes, F. Brochard-Wyart, and D. Quere, Capillarity and Wetting Phenomena. New York: Springer-Verlag, 2004, p. 37.

[44] "RT/duroid ${ }^{\circledR}$ 5870/5880 High Frequency Laminates," Rogers Corporation, p. 2. 